Portland State University

PDXScholar

12-4-1996

\title{
The Multiple Pool Migrating Worker Paradigm : A Distributed System Framework and Model
}

Cynthia Ann Stanley

Portland State University

Follow this and additional works at: https://pdxscholar.library.pdx.edu/open_access_etds

Part of the Electrical and Computer Engineering Commons Let us know how access to this document benefits you.

Recommended Citation

Stanley, Cynthia Ann, "The Multiple Pool Migrating Worker Paradigm : A Distributed System Framework and Model" (1996). Dissertations and Theses. Paper 5831.

https://doi.org/10.15760/etd.7702

This Thesis is brought to you for free and open access. It has been accepted for inclusion in Dissertations and Theses by an authorized administrator of PDXScholar. Please contact us if we can make this document more accessible: pdxscholar@pdx.edu. 


\section{THESIS APPROVAL}

The abstract and thesis of Cynthia Ann Stanley for the Master of Science in Electrical and Computer Engineering were presented December 4, 1996, and accepted by the thesis committee and the department.

COMMITTEE APPROVALS:
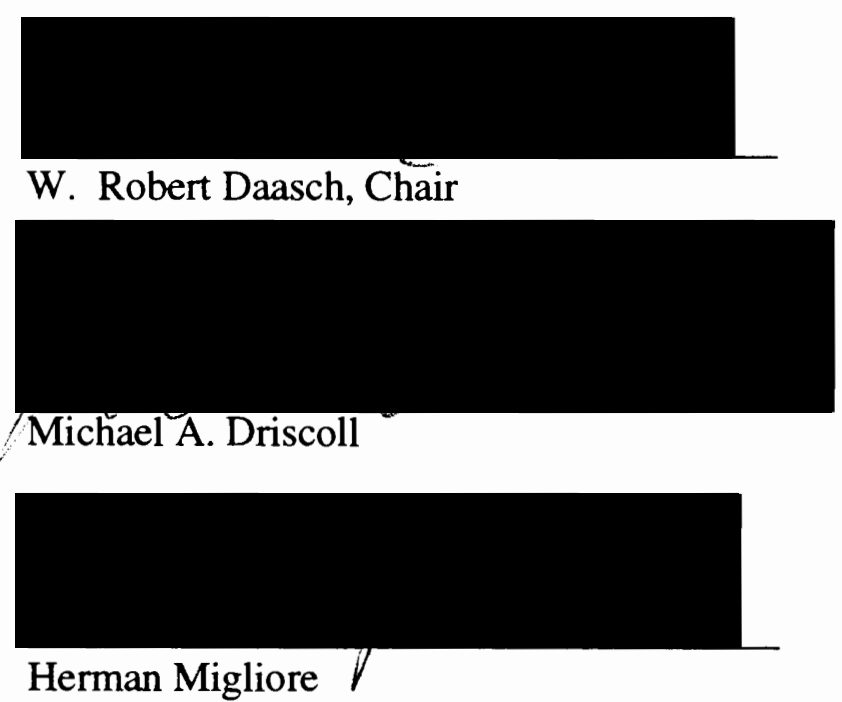

Representative of the Office of Graduate Studies

DEPARTMENT APPROVAL:

Rolf Schaumann, Chair

Department of Electrical Engineering

ACCEPTED FOR PORTLAND STATE UNIVERSITY BY THE LIBRARY

by

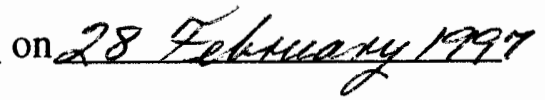


AN ABSTRACT OF THE THESIS OF Cynthia Ann Stanley for the Master of Science in Electrical and Computer Engineering presented December 4, 1996.

Title: The Multiple Pool - Migrating Worker Paradigm: A Distributed System Framework and Model

A network of workstations (NOW) can provide an inexpensive and effective distributed processing platform. The purpose of this thesis is two-fold, providing a methodology for distributed computing on a NOW first, and providing a model to predict and monitor performance second. The Multiple Pool-Migrating Worker (MPMW) paradigm uses multiple job pools to divide up tasks and migrating workers to balance the work load. The MPMW paradigm is a quick and efficient way of implementing problems using distributed processing without extensive knowledge of parallel programming. A model describing the MPMW paradigm is developed using queuing theory and Mean Value Analysis techniques. The model connects run time, granularity and scalability. It is designed to allow prediction of run time from system measurables. The experimental results show that the model describes a system implementing the MPMW paradigm and allows estimation of resources such as the run time in systems with multiple processors.

The paradigm and its model are used to examine the task granularity verses scalability trade-offs in a system. The experimental application used is a serial LU decomposition. There are many overhead costs involved in using a NOW for distributed processing, the more workstations, the more overhead, the less performance. However, the results 
show that the granularity of the tasks in the job pools can affect system performance. It is further shown that scalability can be manipulated by changing the granularity of the job pools. Both task size and bundle size affect performance in distributed systems and the MPMW model presented in this thesis predicts this general behavior. However, the impact of non-optimal task sizes is minimized with migrating workers and job pool bundles. 
THE MULTIPLE POOL - MIGRATING WORKER PARADIGM:

A DISTRIBUTED SYSTEM FRAMEWORK AND MODEL

by

CYNTHIA ANN STANLEY

A thesis submitted in partial fulfillment of the requirements for the degree of

\author{
MASTER OF SCIENCE \\ in \\ ELECTRICAL AND COMPUTER ENGINEERING
}

Portland State University

1997 


\section{Acknowledgments}

I would like to thank my advisor, Dr. Rob Daasch, for his support and guidance during this thesis. His encouragement and creative explanations helped make this work a success.

I want to thank Travis Bonifield for all his wonderful assistance on this project and his emotional support throughout the process.

I wish to thank the faculty and staff of the Electrical Engineering department for their encouragement and assistance throughout not only my thesis, but throughout my graduate career.

My gratitude also to Dr. Henry Crockett and Jeff Able of the School of Business Administration for the financial help of a Graduate Assistantship and the emotional help of friendship. Their support and interest brightened my work.

Most of all, my thanks goes to my husband, Ron Stanley, for his never-ending encouragement, proof-reading, critiques, and questions. Without his support, this work would not have been such a success. 


\section{Table of Contents}

Acknowledgments

PAGE

iii

List of Figures

ix

CHAPTER

1. Introduction 1

1.1. Distributed Computing 1

1.2. Scalability verses Granularity 3

1.3. The Job Pool Paradigm 5

1.4. Fork and Join Primitives 7

1.5. Queuing Theory 8

$\begin{array}{ll}\text { 1.5.1. Introduction } & 8\end{array}$

1.5.2. Related Work 9

$\begin{array}{ll}\text { 1.6. } & \text { Related Work } \\ & 13\end{array}$

1.6.1. Model Parameters 13

1.6.1.1. Number of Processors $\quad 13$

$\begin{array}{ll}\text { 1.6.1.2. Problem Size } & 14\end{array}$

$\begin{array}{ll}\text { 1.6.1.3. Overhead } & 16\end{array}$

$\begin{array}{ll}\text { 1.6.1.4. Load Balancing } & 17\end{array}$

1.6.1.5. System Usage on Non-Dedicated Systems 19

1.6.1.6. Heterogeneity 21

1.6.1.7. Assigning Tasks to Specific Processors 23 
1.6.2. Performance Metrics 23

1.6.2.1. Execution Time 23

1.6.2.2. Speed 25

1.6.2.3. Speedup 26

$\begin{array}{ll}\text { 1.6.2.4. Efficiency } & 27\end{array}$

1.6.2.5. Scalability 28

1.6.2.6. Concurrency 30

1.6.2.7. Granularity 30

1.6.3. Selecting Metrics and Parameters 31

1.7. Summary 32

2. Theoretical Basis 33

2.1. The Job Pool Paradigm 33

2.1.1. Structural Primitives 33

2.1.1.1. Job Pool Primitives 34

2.1.1.2. Worker Primitives 35

2.1.1.3. Job Pool Management 36

2.1.2. Transformational Primitives 38

2.1.3. Operational Primitives $\quad 41$

2.2. Model Derivation 42

2.2.1. System Variables 42

2.2.2. Model Derivation 43 
2.2.2.1. Defining Work 44

2.2.2.2. Total Run Time with a Single Job Pool 46

2.2.2.3. Total Run Time with Multiple Job Pools 47

$\begin{array}{ll}\text { 2.2.2.4. Expanding } T_{s} \text { and } T_{p} & 51\end{array}$

2.2.2.5. Expanding Q(N-1) 53

2.2.2.6. Limit Analysis of Run Time 55

2.2.3. Amdahl's Law and Run Time 58

$\begin{array}{ll}\text { 2.2.4. Speedup } & 59\end{array}$

2.2.4.1. Limit Analysis of Speedup 60

$\begin{array}{ll}\text { 2.2.5. Granularity } & 61\end{array}$

2.2.5.1. Limit Analysis of Granularity 64

$\begin{array}{ll}\text { 2.2.6. Scalability } & 65\end{array}$

2.2.6.1. Limit Analysis of Scalability 66

2.2.7. Efficiency 66

2.2.7.1. Limit Analysis of Efficiency 67

$\begin{array}{ll}\text { 2.2.8. Summary } & 68\end{array}$

2.3. Multiple Pool, Migrating Worker Implementation 68

$\begin{array}{ll}\text { 2.3.1. Physical Framework } & 68\end{array}$

$\begin{array}{ll}\text { 2.3.2. System Measurements } & 72\end{array}$

$\begin{array}{ll}\text { 2.4. Summary } & 73\end{array}$ 
3. Applications $\quad 75$

3.1. Characteristics for Mapping to the MPMW Model 75

3.2. Selected Examples $\quad 78$

$\begin{array}{ll}\text { 3.2.1. Plasma Simulation } & 78\end{array}$

3.2.2. Genetic Database Alignment 81

3.3. Detailed Example: LU Decomposition with Back Substitution 83

\begin{tabular}{ll} 
4. & Results and Discussion \\
\hline 8
\end{tabular}

4.1. System Usage 90

4.2. Phase 1 - Equivalent Job Pools 93

4.2.1. System Measurements 93

4.2.1.1. Execution Times 94

4.2.1.2. Task Computation Time and te 98

4.2.1.3. Resource Busy Time 100

4.2.1.4. Spinlocks 103

$\begin{array}{ll}\text { 4.2.1.5. Scalability } 108 & 108\end{array}$

4.2.2. Experimental Data verses Models 109

4.3. Phase 2 - Uneven Job Pools 112

$\begin{array}{ll}\text { 4.3.1. System Measurements } & 112\end{array}$

4.3.1.1. Execution Times 113

4.3.1.2. Task Computation Time and te 114

4.3.1.3. Resource Busy Time 116 
viii

4.3.1.4. Spinlocks

121

4.3.2. Experimental Data vs. Models

128

5. Conclusions and Future Work

133

5.1. Conclusions

133

5.2. Future Work

136

References

139

Appendices

145

$\begin{array}{ll}\text { A. Job Pool Pseudocode } & 145\end{array}$

B. Experimental Program Runs 


\section{List of Figures}

\section{FIGURE}

PAGE

1-1a. Forking Tasks

1-1b. Joining Tasks

1-2a. Job Pool Fork $\quad 8$

1-2b. Job Pool Join $\quad 8$

2-1. Basic Job Pool Setup 34

2-2. Transformational Primitives 38

2-3. Gantt Chart 45

3-1. Job Pool Representation of Pipelining and Parallel Execution $\quad 77$

3-2. Possible Movements of Particles among Job Pools 80

4-1. Flotsam on Wednesdays 90

4-2. System Usage on Wednesdays 91

4-3. N vs. T, 50x50 Matrices 95

4-4. $\quad \mathrm{N}$ vs. G vs. T, 50x50 Matrices 96

4-5. $\quad \mathrm{N}$ vs. T, 100x100 Matrices 97

4-6a. Task Computation Time, 50x50 Matrices, $\mathrm{G}=20 \quad 98$

4-6b. Task Computation Time, 100x100 Matrices, G=20 99

4-7a. Resource Busy Time, 50x50 Matrices, $G=20 \quad 100$

4-7b. Resource Busy Time, 100x100 Matrices, G=20 100

4-8. N vs. G vs. B, 50x50 Matrices 102 
4-9a. Spinlocks, $50 \times 50$ Matrices, $G=20$

104

4-9b. Spinlocks, $100 \times 100$ Matrices, $G=20$

104

4-10. $\mathrm{N}$ vs. $\mathrm{G}$ vs. Vs, $50 \times 50$ Matrices

105

4-11a. Spinlock Count vs. Program Execution, N=11, G=1

106

4-11b. Spinlock Value Distribution, $N=11, G=1$

107

4-12a. $\mathrm{T}$ vs. $\mathrm{N}$ for $\mathrm{G}=1$

108

4-12b. $\mathrm{T}$ vs. $\mathrm{N}$ forG $=10$

108

4-12c. $\mathrm{T}$ vs. $\mathrm{N}$ for $\mathrm{G}=30$

109

4-12d. Tvs. $\mathrm{N}$ for $\mathrm{G}=50$

109

4-13a. Model vs. Actual: N vs. T, 50x50 Matrices, $G=20$

4-13b. Model vs. Actual: $N$ vs. T, $100 \times 100$ Matrices, $G=20$

4-14. Uneven Pools: N vs. T, 50x50 Matrices

4-15a. Uneven Pools: Task Computation Time, $\mathrm{G}_{\mathrm{A}}=1$

4-15b. Uneven Pools: Task Computation Time, $G_{B}=50$

4-16a. Uneven Pools: Resource Busy Time, Pool A, $G_{A}=1$

4-16b. Uneven Pools: Resource Busy Time, Pool B, $\mathrm{G}_{\mathrm{B}}=50$

4-17a. Uneven Pools: Resource Busy Time, Pool A, $G_{A}=50$

4-17b. Uneven Pools: Resource Busy Time, Pool B, $G_{B}=1$

4-18a. Uneven Pools: Vs to Get Tasks from Pool A, $\mathrm{G}_{\mathrm{A}}=1$

4-1 8b. Uneven Pools: Vs to Put Tasks in Pool $B, G_{B}=50$ 
4-19a. Uneven Pools: Vs to Get Tasks from Pool $A, G_{A}=50$

4-19b. Uneven Pools: Vs to Put Tasks into Pool B, $G_{B}=1$

126

4-19c. Uneven Pools: Vs to Get Tasks from Pool B, $\mathrm{G}_{\mathrm{B}}=1$

127

4-20. Comparing Model verses Actual Data, $G_{A}=1, G_{B}=50$

129

4-21. Comparing Model verses Actual Data, $G_{A}=50, G_{B}=1$

130 


\section{Chapter 1}

\section{Introduction}

\subsection{Distributed Computing}

Computers have become computationally powerful over the last couple of decades and will continue to improve. However, as computer power increases, program complexity and problems requiring computation grow as well. Architectures involving one or more processors are continuously evolving to meet the computational needs of today's algorithms. One multiprocessor architecture is based on distributed computing. Programs dealing with molecular sequence analysis [1], plasma simulation [2], or circuit optimization [3] can involve long execution times on a single processor. Algorithms such as these, however, lend themselves to distributed processing, because multiple processors can be used to compute separate parts of the problem concurrently, thus reducing overall execution time to a more practical level. Many architectures exist that consist of a single system with some number of processors connected by specialized high speed connections (supercomputer). While these machines are very useful for the types of problems mentioned, they are also expensive. Unbeknownst to many potential users, most already have access to an inexpensive and effective alternative to supercomputers: a Network Of Workstations or NOW $[1,3,4,5,6]$.

Networked workstations can provide an inexpensive parallel processing platform. A NOW can be visualized as a single virtual machine with some number of processors, in 
the form of workstations, connected by local area network (LAN), wide area network (WAN) or even the Internet [1]. While supercomputer performance should not be expected for many applications, it is still possible to get significant performance gain using a NOW over a serial implementation. There are several fundamental differences between networked workstation processors and the multiple processors in a supercomputer. First of all, the communication between processors is completely different. The processors in a NOW are connected to a LAN or WAN loaded with other miscellaneous traffic while the processors of a supercomputer are at most a few inches apart with dedicated high speed connections. Even though LAN's (or WAN's) will never achieve the speed of a supercomputer's connectivity, LAN technology is getting faster with the advent of such technologies as ATM, FDDI, and 100 megabit-per-second Ethernet [7]. Secondly, the processors in a multiprocessor machine are usually all the same, i.e. homogeneous, whereas a network can be composed of a variety of machines with varying computing power, i.e. heterogeneous. Third, networked machines are almost never totally dedicated to a single problem, whereas at least a subset of the supercomputer's processors usually are. The owner of the workstation as well as other users can put a load on a machine, leaving the idle cycles for use by a distributed process $[4,8-12]$.

The presence of these idle cycles is another advantage of the NOW. Even using the idle cycles of processors in a non-dedicated network, superior performance can be obtained over a sequential implementation, with relatively little extra cost in equipment or 
dedicated computer time. A non-dedicated network is one shared by many users for multiple applications, thus a distributed application could run during the idle cycles on other users machines in a pre-existing network. A potential cost, however, in attempting to use any distributed computing environment is converting the sequential algorithm to a parallel one. Many who would benefit from distributed processing may not be prepared to write parallel programs, therefore some interface or framework is needed to help users gain the benefits of distributed programming without the costs of learning to program in parallel. This framework should also give a novice distributed programmer an idea of how the program will work in parallel, how efficient it will be on a system, and how it will scale with the number of workstations available. The object of this thesis is to provide such a framework.

\subsection{Scalability verses Granularity}

While the literature has no single established definition for scalability, it is popularly accepted as the ability of a system's performance to increase with respect to increasing problem size or system size $[13,14]$. A system which scales well can have linearly increasing speedup as the problem size and/or system size grows. Scalability can be difficult to visualize as both the problem size and the system change, because it is how these two factors affect system performance together that signifies scalability. If the overhead cost incurred by increasing the number of processors (or workstations) used for a particular program increases too rapidly, then likely the system scales poorly for 
that program. As more is learned about how a system scales the performance of a large problem on a large system can be predicted using a small problem on a small system.

One possible method to alter a program for distributed processing is to break it up into individual tasks or bundles of tasks that are ultimately distributed to the processors for computation. The granularity is how large a task, or how many tasks, a single processor in a loosely-coupled system of processors handles at a time. A NOW is an example of a loosely-coupled system of processors; the processors can be far apart and work independently. Increasing the task size, or number of tasks in many cases, reduces the communication costs incurred in distributed execution. The communication costs that accumulate include getting the tasks to a workstation and removing them after computation. Unfortunately, most of the time increasing the task size, or number of tasks taken at a time, also decreases the parallelism inherent in a distributed system. As the amount of work an individual processor receives increases, the potential for spreading that work among more processors decreases, thus preventing load balancing over those processors. Therefore granularity presents serious questions about the tradeoff of communication costs, parallelism and load balancing. Strumpen [1] points out that the "granularity of the tasks determines the efficiency of the computation." Indeed, increasing the task size can upset the load balance, decreasing efficiency, though as Karp and Flatt [15] point out, it is possible to be content with a lower efficiency depending on the circumstances of time and cost. Decreasing task size can also reduce 
efficiency, however, by increasing the overhead involved in having to retrieve tasks more often.

The tradeoff presented by granularity is that it affects the scalability. If changing the granularity changes the overhead costs enough, then the scalability of the system may be altered. Increasing the granularity of a problem reduces some communication costs since the processors have to make fewer "trips" to retrieve more work to do. This reduction in costs could increase the scalability of the system since it is the overhead costs that limit scalability in the first place. Because of the influence of granularity on the system, it could also heavily affect the system's scalability. The paradigm presented in this thesis gives a potentially useful technique for increasing the scalability of poorly scaleable systems, making such systems better candidates for distributed computing.

\subsection{The Job Pool Paradigm}

In this thesis the job pool paradigm [3] is used to apply distributed programming to coarse grained applications. In such applications the work is divided into tasks which are kept in a job pool. The processors that work on these tasks are dubbed workers; the workers go to the job pool for tasks to compute. A task is comprised of work from the program; it can contain multiple units of work rather than a single piece. When the worker finishes a task, it places the results in the next job pool, then goes back to the original pool for the next task to work on. This basic paradigm was introduced by Schaefer [3] and provides a simple means of dynamic load balancing. Each worker 
proceeds at its own pace and gets additional work only when it is done with what it has, thus the computational load is spread out by the workers themselves. A particularly slow processor will not become a bottleneck, it will simply do less overall work. The idea of using a job pool or work pool for dynamic load balancing can also be seen in the work by Daasch et. al [16] and Strumpen [1] as well as Schaefer[3].

Schaefer [3] suggested that her work could be extended to include multiple job pools for the implementation of complex jobs with several different kinds of tasks. In this work, the ideas presented by Schaefer are expanded to include a system of two (or more) job pools rather than just one. Beyond the work by Schaefer, is the new idea of migrating workers. Since there are multiple job pools, there can be multiple sets of workers. To increase efficiency the workers are permitted to move from one job pool to another, changing the type of work they perform to match their current job pool.

The job pool paradigm is defined by a series of primitives. These primitives were categorized as structural, operational, or transformational by Schaefer [3]. The structural primitives describe the workers and the job pool. The transformational primitives are a series of primitives based on the fork and join ideas. These primitives include the one-to-one transformation, the one-to-many transformation, the many-to-one transformation and the many-to-many transformation. As this thesis works with two job pools, these primitives can be combined to form compound transformation primitives. Section 2.1 contains an extended discussion of the job pool paradigm and its primitives. 


\subsection{Fork and Join Primitives}

The general idea of forking and joining can be seen in several papers in the literature $[16,17,18,19,20]$ and is expressly discussed by Bacelli and Lui and Bacelli et al in $[21,22]$. The act of forking is when one item becomes several items and joining is when several items become one. Specifically, Bacelli et al. describe a fork as the creation of new customers sent to different queues and a join as what happens after all these new customers are serviced. There are two possible implementations of forking and joining in relation to the work in this thesis. As section 2.1 later describes, there are a variety of transformational primitives that are defined by the fork and join primitives. In the oneto-many transformation, for example, a worker may take one task from the pool it serves, complete the associated computation with the information for that task, and end up with multiple result tasks to place in the next pool. Thus one task is forked into several, as shown in figure 1-1a. In figure 1-1b, a join is shown, where several tasks are taken from one job pool and combined during computation to form a single output task for the next job pool.

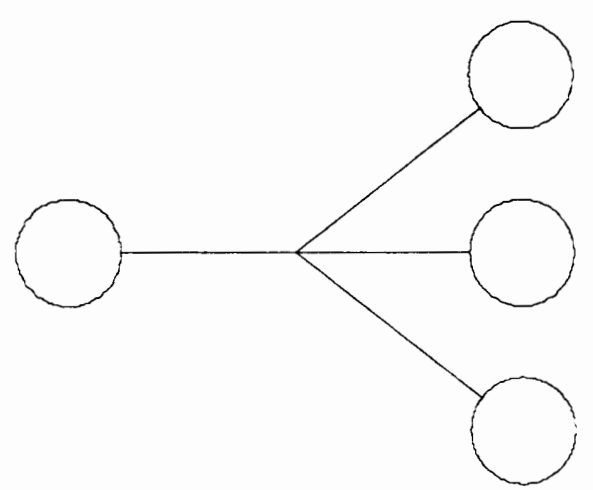

Figure 1-1a. Forking Tasks.

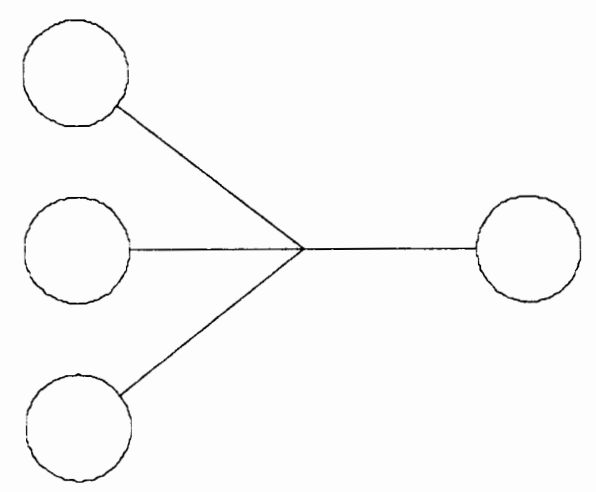

Figure 1-1b. Joining Tasks. 
The second possible implementation would be an interesting extension of the work presented here and is thus an issue for further work as discussed in chapter 5. The extension allows the forking and joining of job pools rather than tasks. Looking at the one-to-many transformation example, the forking could be extended to where some of the resulting multiple tasks go to one job pool and the rest to others, (Fig 1.2a) thus implementing a job pool fork. Conversely a worker could retrieve tasks from two different job pools to combine in computation and deposit as a single task in the next job pool, (Fig 1.2b).

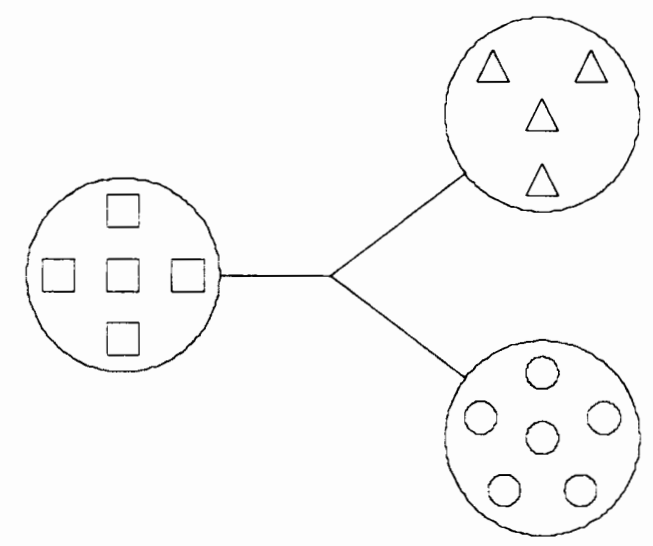

Figure 1-2a. Job Pool Fork.

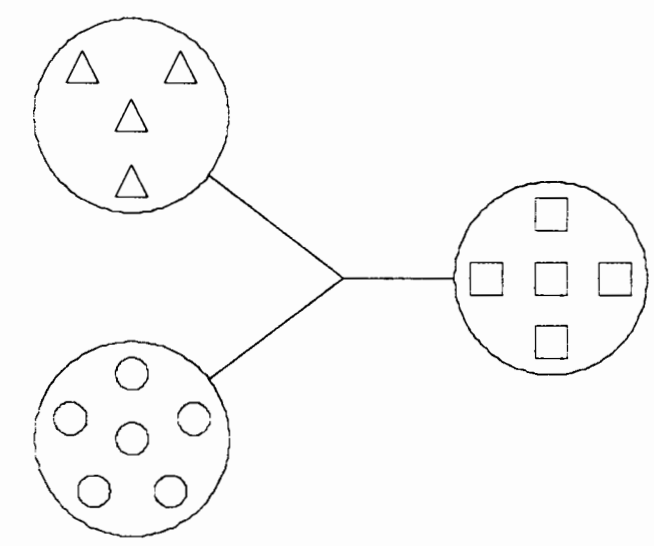

Figure 1-2 b. Job Pool Join.

\subsection{Queuing Theory}

\subsubsection{Introduction}

According to Kleinrock [23], queuing theory is the study of "the phenomena of standing, waiting, and serving." Whether a job is standing in line for disk access, or a task is waiting for service from a processor, modeling the behavior of computer 
communications networks is a natural extension of queuing theory $[23,24]$. Kleinrock [23] presents a description of a computer network as a single large resource (server) with a single queue forming the job stream (service center) for waiting jobs. Kleinrock then compares a $\mathrm{M} / \mathrm{M} / \mathrm{m}$ queuing model to a more general $\mathrm{G} / \mathrm{G} / \mathrm{m}$ queuing model for describing the performance of the system. He finds the $\mathrm{G} / \mathrm{G} / \mathrm{m}$ model more suitable and uses $\mathrm{m}$, the scaling factor, to improve the mean response time of the system. Rather than studying distributions, Lasowska et al. [24] use Mean Value Analysis (MVA) of their queuing models to describe a computer network and determine response time from the average behavior of the system. This approach is also referred to as queuing network theory. The work in this thesis uses MVA as presented by Lasowska et al. rather than the distribution driven queuing models of Kleinrock. This keeps the models simple to evaluate and understand.

\subsubsection{Related Work}

Both queuing and MVA models have been extended to describe these distributed systems. Kleinrock and Huang [25], for example, model a parallel processing system as a single queue of waiting jobs with a central resource consisting of a number of processors. Only one job is executed at a time using its optimal number of processors. If a job's optimal number of processors is greater than the number of processors actually available, then some processors must execute more than one task and execution time increases. If there is an excess of processors, some sit idle and efficiency decreases. Kleinrock and Huang present an expression to find the optimal number of processors to 
maximize a metric they call power, which minimizes execution time while maximizing efficiency. Simply stated, power is equal to throughput divided by response time. The optimal number of processors to maximize power is given by:

$$
P^{*}=\frac{\beta}{r \alpha}
$$

where $\beta$ is the summation of a vector describing the fraction of tasks in a job that can use $\mathrm{P}_{\mathrm{i}}$ processors, $\alpha$ is the summation of the fraction vector divided by the processor vector containing $\mathrm{P}_{\mathrm{i}}$, and $\mathrm{r}$ is a system parameter. When power is maximized, the system contains an average of one job. Kleinrock and Huang's model uses a M/G/1 queuing system to find average response time in the system with new arrivals using mean service time, system utilization, and the coefficient of variance for the service time distribution. In a system without arrivals, the mean service time is used.

In contrast, Heidelberger and Trivedi [20] use MVA models to examine parallelism within jobs in their system. While multiple jobs execute concurrently, parallelism within jobs is allowed via multitasking. Tasks in the system visit a series of nodes; a visit to node 0 splits the task into primary and secondary tasks. The average number of visits at a node $i$ verses visits to node 0 and the average service time requirements per visit to node $i$ are used to describe system behavior in their models. Heidelberger and Trivedi find that due to the parallelism, their models do not have exact solutions, thus they present a method to find approximate solutions. Using this methodology, 
Heidelberger and Trivedi look at the performance improvement from overlapping I/O time with computation time.

Nelson et al. [19] use a $M^{\mathrm{x}} / \mathrm{M} / \mathrm{c}$ queuing system to model a centralized parallel processing system. They then compare their centralized model with other model configurations. This work, like Kleinrock's [23], compares a central queue feeding all processors to a distributed queue where each processor is fed by its own queue. Nelson et al. go on to make a second comparison between job splitting, where tasks of the same job enter different queues (processors), and non-splitting, where all tasks of a job are executed by a single processor. Job splitting reflects parallelism, whereas the nonsplitting case describes multitasking. Kleinrock describes a computer communication network rather than a distributed system and thus uses the non-splitting case. Nelson et al. model mean response time as the sum of the job waiting time and the job service time, similar to Kleinrock. The job waiting time reflects the amount of time a job must wait in the queue before the first of its tasks is started. The job service time is the processing time for all tasks in a job. The results of Nelson et al. show that a centralized system with job splitting has the lowest mean response time. Using a distributed queue system with no splitting had the worst response time. The relative response times of the distributed system with job splitting and a non-splitting centralized system depended on the system utilization. For low to moderate system utilization the distributed system with splitting had a lower mean response time, but when system utilization was high the non-splitting centralized case had the lower time. The work by Kleinrock also favored 
the centralized queue model over a distributed one, though he dealt with the nonsplitting case only. Also, Kleinrock decided to use the more general $\mathrm{G} / \mathrm{G} / \mathrm{m}$ model to relax the assumptions made about the statistical behavior of the system by the $\mathrm{M} / \mathrm{M} / \mathrm{m}$ model.

Like the work by Kleinrock and Nelson et al., this thesis uses a central queue (the job pool) to service all the processors (workers). Nelson's job splitting verses not splitting presents the question of task granularity, a major concern of this thesis. Rather than using queuing theory, however, queuing network theory, similar to Heidelberger and Trivedi, is used. The two approaches are similar and similar expressions for service residence times in the service centers result. Task partitioning is ignored, as in the work of Kleinrock and Huang [25], as the model in this thesis lets processors partition the tasks among themselves dynamically; as each processor finishes a task, it returns to a job pool for another one.

Another queuing theory model, Synchronized Queuing Networks (SQN) developed by Baccelli and Liu [21], represents each processor in a system as a single server queue. Tasks or customers are assigned with precedence to these processor queues and are executed in first come first serve (FCFS) order after its predecessors have been completed. System communications are also modeled as customers served by single server queues. The SQN are a generalization of the Acyclic Fork/Join Queuing Networks presented by Baccelli et al. [22]. The fork primitive describes the creating or 
spawning of several new tasks which are then sent to different queues. The join primitive reverses this process when all the forked tasks are completed. The fork and join primitives are an integral part of the paradigm presented in this thesis.

\subsection{Related Work}

There are a large number of models attempting to describe the behavior of distributed and parallel systems. While these models vary widely, there are a basic set of parameters and metrics that are used to construct these models. Rather than discuss each of the various models, some of the basic parameters and performance metrics seen in these models are discussed and examples of how they are used cited. The parameters and metrics given here are not an all inclusive listing, but is a representation of those seen in other work. A given model may not use all these parameters and metrics, or may use additional ones.

After examining the work in the literature, those metrics and parameters necessary for a good model will be chosen and then used as a basis for forming the Multiple Pool, Migrating Worker (MPMW) model.

\subsubsection{Model Parameters}

\subsubsection{Number of Processors}

The number of processors is the most obvious factor that affects the performance of a parallel program. Amdahl's Law shows the direct influence of the number of 
processors on a system. Amdahl's Law, a basis for many parallel performance models, can be used to define speedup in general terms [23] as

$$
\text { Speedup }=\frac{T_{1}(1)}{T_{N}(N)}
$$

Speedup is essentially a measure of how much faster one system implementation is over a another system implementation. This performance metric will be discussed further in 1.6.2.3.

In this thesis, the speedup of a system with multiple processors $(N)$ over a system with a single processor is examined. The number of processors is obviously the reason for performance gain and is an integral part of every model.

\subsubsection{Problem Size}

The size of the problem being solved can have a drastic affect on the performance. Simply put, a program adding two $2 \times 2$ matrices will execute much faster than a program adding two $1000 \times 1000$ matrices. There are two ways problem size is used in relation to models found in the literature, with execution time and with scaling. Scaling is the ability of a problem to grow linearly with respect to the computing resources available. A good parallel model needs to take problem size into account, and in fact, most models use problem size as a pivotal factor in performance measurement.

Several types of descriptions are used to incorporate program size into performance models. Zhang and Yan [4,5] denote the size of a program by the term $|\mathrm{A}(\mathrm{I})|$ where $\mathrm{A}(\mathrm{I})$ 
is the parallel program and I reflects the input parameter. The model presented by Peterson and Chamberlain [28] incorporates problem size by looking at the number of “events" per iteration, where a program or algorithm runs for I iterations. Donaldson et al. [18] use a task graph to model a program as a series of $n$ tasks where $G_{p}=(V, E)$ and $\mathrm{V}=\{\mathrm{t} 1, \mathrm{t} 2, \ldots . . \mathrm{tn}\}$. Baccelli and Liu [21] also follow the task graph approach. Driscoll and Daasch [29], expanding on work by Amdahl and Gustafson [27], present a formulation of Amdahl's Law:

$$
T(p, w)=\operatorname{Seq}(p, w)+\frac{\operatorname{Par}(p, w)}{p}
$$

where $\operatorname{Seq}(p, w)$ is the time to execute strictly sequential code, $\operatorname{Par}(p, w)$ is the time to execute the fully parallel portion of the code, $p$ represents the number of processors in the system, and $w$ is the work. This equation explicitly looks at the effects of problem size on the sequential and parallel execution times. Some performance models, like Zhang and $\mathrm{Xu}$ [30], model problem size simply as some value W. Similarly, Nussbaum and Agarwal [14] give problem size as $s$, a function of the encoding of the input. The generic $W$ was defined by Zhang et al. [31] as the "number of basic operations needed by the fastest known sequential algorithm to solve the problem on a sequential computer." Grama et al. [32] also use this definition for problem size. A similar approach was taken by Kleinrock and Huang [25], who look at problem size as how long it takes to execute on a single processor. 


\subsubsection{Overhead}

Overhead is generally used to denote the total time used by a parallel program to set up the various parallel processes which need to be run, the communication between processors during the parallel execution time, and any extra time needed to synchronize the processes at the various points of a parallel routine iteration. This factor is particularly important when one is discussing parallel programs run on a NOW platform, as the communication time between processors can be, and often is, the greatest bottleneck to total runtime.

Amdahl's Law and some subsequent models $[14,15]$ bury the overhead term in either the parallel time or the serial time coefficients. Some authors choose to ignore overhead and communications to simplify their models $[8,25]$ while others use a single overhead term, " $T_{0}$ ", such as Grama et al. [32]. Donaldson et al. [18] ignore intramachine communications in networked multiprocessor machines, and assumes other communications times are constant.

Other models go to great lengths to model the effects of overhead terms on performance. These models tend to be designed for networked workstations rather than parallel machines, since overhead, especially communication time, is more of a problem in systems that do not share a single box. Peterson and Chamberlain [28] model runtime performance as:

$$
R_{P}=I *\left(t_{\text {comp }}+t_{\text {comm }}+t_{\text {ovhd }}+t_{\text {sync }}\right)
$$


where $\mathrm{I}$ is the number of iterations, $\mathrm{t}_{\text {comp }}$ is the CPU computation time, and $\mathrm{t}_{\text {comm }}, \mathrm{t}_{\mathrm{ovhd}}$, and $\mathrm{t}_{\text {sync }}$ individually model parts of overhead; communications, setup overhead, and synchronization time, respectively. The synchronization time models the time that is wasted waiting for all tasks to complete before continuing. Zhang and his coresearchers use overhead to define a latency metric $[4,5,30,31,33]$ :

$$
L(A(I), H N)=\frac{\sum_{j=1}^{m} \text { overhead }(j) W_{j}}{\sum_{j=1}^{m} W_{j}}
$$

with $\mathrm{m}$ equal to the number of processors. $\mathrm{L}(\mathrm{A}(\mathrm{I}), \mathrm{HN})$ is average latency, $\mathrm{W}$ is a weighting factor comparing a processor to the system's fastest processor, the overhead is total execution time minus the owner's time usage and the computation time. For completeness, it should be noted that some models can consider the overlapping of the communications overhead and the computation time. Mechosa et al. [34] follow this strategy.

\subsubsection{Load Balancing}

Load balance is the degree to which multiple processors in a parallel system are in use during a given interval of time. A program is broken up into tasks that can be executed in parallel and these tasks must somehow be assigned to the processors. There are three possible situations that arise. The first possibility is that there are fewer tasks than processors, in which case some processors sit idle, lowering system efficiency. Removing the extra processors from the system simplifies the first possibility into the 
second case, where there are as many tasks as processors. This would reflect a possible perfect load balancing, one task per processor. In general, assuming an equal number of tasks and processors is a simplification many models $[4,34]$ use to assume load balancing. The final case occurs when the number of tasks exceed the number of processors. In this situation some sort of load balancing is performed to spread the work among the processors such that no processor becomes a serious bottleneck, slowing overall execution. Another complicating factor arises when the tasks are not equivalent in size. For example, if there are the same number of tasks as processors, but the tasks vary greatly in size, then a load imbalance would be seen.

Load imbalances can greatly affect performance [28]. Load balancing can be done, with some restrictions, statically or dynamically (on the fly). Some authors $[5,18,21]$ assume static assignment of tasks to processors to simplify their model, others attempt to take the dynamic nature into account. Donaldson et al. [18] define a value, $\alpha$, as a measure of how well a static assignment balanced the load on the workstations. Grama et al. [32] describe how their isoefficiency metric can be used with dynamic load balancing schemes of other researchers. Peterson and Chamberlain [28] model dynamic load balancing as does Schaefer in her queuing theory based model [3]. Kleinrock and Korfage [8] neglect the task assignment and load balancing question altogether.

Peterson and Chamberlain [28] found that load balancing to have a significant effect on the parallel performance. The basic model by Peterson and Chamberlain is 


$$
R_{P}=I *\left(t_{\text {comp }}+t_{\text {comm }}+t_{\text {ovhd }}+t_{\text {sync }}\right) \text {. }
$$

The $\mathrm{T}_{\text {comp }}$ term is further broken down to:

$$
\mathrm{t}_{\text {comp }}=\beta \mathrm{N}_{\mathrm{e}} \mathrm{t}_{\mathrm{e}} / \mathrm{P}
$$

where $\beta$ is the load balancing factor, $N_{e}$ is the number of events processed, $t_{e}$ is the event processing time and $\mathrm{P}$ is the number of processors. At this point these authors look in depth at modeling the load imbalance using probability statistics with triangle and Bernoulli distributions and order statistics.

The queuing theory approach uses the idea of putting tasks in queues for the processors to model load balancing and overhead from networking. Schaefer's [3] model allows the number of tasks to be greater than the number of processors. The tasks are placed in a "pool of tasks" and a number of "workers" are started up on participating workstations. Idle workers request tasks from the pool of tasks, execute that task, then return for another. Only one worker at a time can access the pool of tasks. This load balancing scheme is the basis of Schaefer's model and is modeled using queuing theory to produce a result similar to the Erlang B (loss) formula seen in telephone switching models [23].

\subsubsection{System Usage on Non-Dedicated Systems}

Unlike a dedicated system, a NOW platform is likely to have an existing load when a distributed program begins. When using a network of workstations, one is sharing computing resources with the owners of the individual machines and other transient 
users. While a single supercomputer is usually dedicated to solve a problem, there's not much chance that an entire network of workstations will be used exclusively to solve a single parallel program. Some models ignore this factor and look at dedicated systems only. Most performance models for massively parallel machines take this approach. When dealing with a network of workstations this simplification is not so easily made as owner utilization of the individual machines affects performance $[5,8]$.

The work by Kleinrock and Korfhage [8] is based on using the "extra" cycles beyond owner utilization. Distributions describing a processors use is split into available time, $A(t)$, and unavailable time, $N(t)$, portions. These distributions are used to determine the distribution of a program's finishing time, $f_{\text {ave }}$. A simple model is defined as:

$$
f_{\text {ave }}=\frac{t_{a}+t_{n}}{t_{a} M} * W
$$

where $t_{a}$ is the time that the processor is available, $t_{n}$ is the unavailable time, $\mathrm{W}$ is amount of work in minutes, and $M$ is the number of processors. The authors then try using a more comprehensive model utilizing the Brownian Motion Approximation, they achieve the same $f_{\text {ave }}$ but have a different variance. The authors look at a variety of distribution functions.

Zhang and Yan [4,5] model networks of heterogeneous, non-dedicated workstations, therefore modeling owner utilization is prominent in their work. In [4], owner workload is incorporated into an overhead term used to define their latency metric in equation (1). In [5], a discrete time model is used to simulate owner workload. A probability of an 
owners task arriving during a time step and how long that task takes to execute are incorporated into a model of the execution time of a "computational segment" at a single machine.

Leutenegger and Sun [11] define a metric called a task ratio to look at the feasibility of distributed processing in terms of system utilization. They define the task ratio as the demand of one parallel task over the time an owner process uses the system. In this case a single parallel task is the total demand of the parallel job divided by the number of workstations used. This task ratio, Leutenegger and Sun maintain, must be sufficiently large to retain most of the possible speedup for the parallel process.

Mutka [12] uses a simple strategy of determining system usage based on the historical system usage for a particular day and a particular portion of that day. Basically, he uses an average of past usages for an hour of a day and compares it to the actual usage at that time to predict the next days usage at the same time. Thus Mutka uses past measurement verses modeling system usage. Celenk and Wang [10] use Mutka's basic theory to construct two thresholds, $\mathrm{L}_{1}$ and $\mathrm{L}_{2}$, with which they construct a state table to determine idle machines whenever task migration occurs.

\subsubsection{Heterogeneity}

A multiprocessor machine generally contains some number of identical processors and is said to be homogeneous. A network of workstations (NOW), however, can be made up of a variety of machines, ranging from the very fast to the very slow in various 
network configurations. A NOW can also have a variety of $\mathrm{V} / \mathrm{O}$ configurations. These systems are said to be heterogeneous. Like owner utilization, heterogeneity is a factor affecting NOW systems, but not traditional supercomputing systems. This factor can and often does greatly affect the performance of a parallel algorithm on a NOW platform.

There is not as much seen in literature directly describing the effects of heterogeneity. In one modeling technique the heterogeneity of a system is accounted for by assigning tasks to the processors best suited to their execution. This is described in section 1.6.1.7. Donaldson et al. [18] have a heterogeneity term buried in their model as $\sum \Delta_{\rho}^{M i}$ as $i$ goes from 2 to $m$, the number of processors. $\Delta_{\rho}^{M i i}$ is the serial execution time of the tasks in $\rho$ assigned to $M_{i}$ by machine $M_{l}$ minus the execution of these tasks by the $M_{i}$ they are assigned to.

The network model used by Zhang and Yan [4] assumes a heterogeneous set of network workstations, differing by the power of it's CPU and the access speed of the I/O and memory. They define heterogeneity as:

$$
H=\frac{\sum_{i=0}^{m}\left(1-W_{i}(A)\right)}{m}
$$

where $\mathrm{m}$ is the number of processors, and $\mathrm{W}$ is a "power weight" describing machine $\mathrm{i}$ compared to the fastest machine in the system. This measure is used in the model to describe the speedup of a NOW. 


\subsubsection{Assigning Tasks to Specific Processors}

Generally, models in the literature assume that the tasks to be done are roughly similar in size and computational complexity. A few research endeavors have included in their models a measure of how well certain types of tasks are given to the processors best suited to process that task. This type of modeling is seen by Mechosa et al. [34] when networking supercomputers together with high speed connections. Donaldson et al. [18] define a value, $\beta$, to give a measure of how specialized a task is then use $\beta$ to discern how useful machines in the system are as far as improving performance by accommodating a specific task. $\beta$ is given as a ratio of the second fastest machine's execution time in a network of machines over the fastest machine's execution time for a task. Donaldson et al. use this measure in their model and their description of speedup.

\subsubsection{Performance Metrics}

\subsubsection{Execution Time}

Total execution time is perhaps of most interest in a distributed system, since cutting runtime is a primary reason for turning to parallel processing in the first place. However, relatively little literature addresses runtime directly, most deal with efficiency, scalability, speedup, etc. However, those that do model runtime directly can be broken up into two groups, those that work with Amdahl's law and those that develop descriptive models from overhead, system usage, etc. 
Amdahl $[27,29]$ was one of the first to model execution time of parallel programs. His work resulted in Amdahl's law

$$
T(p)=T_{\text {serial }}+\frac{T_{\text {parallel }}}{p}
$$

Here the parallel and serial portions of a program are divided into separate parts. Amdahl theorized that as the number of processors approached infinity, the total runtime would approach the serial time. Thus in Amdahl's Law the performance is bound by the serial time. Gustafson $[27,38]$ later elaborated on this and extended the formula to take into account that as the number of processors increases, the problem size often increases as well, bringing scalability into the picture. Gustafson asserted that the problem size is a function of the number of processors and that

$$
T(p)=T_{\text {serial }}+\frac{f(p)}{p}
$$

where $f(p)$ is the problem size expressed in terms of the number of processors. Heath and Worley [29] went further with this and asserted that the parallel time is a function of the problem size and the number of processors

$$
T(p)=T_{\text {serial }}+\frac{g(w)}{p}
$$

where $w$ is the problem size expressed in terms of the number of processors. The serial time that bounds the performance includes the overhead necessary to produce the $g(w) / p$ savings. Daasch and Driscoll [29] take the idea another step and suggest that the serial 
time is also a function of the number of processors and the problem size, as well as the parallel time.

$$
T(w, p)=T_{\text {serial }}(w, p)+\frac{T_{\text {parallel }}(w, p)}{p}
$$

This concept also alters the simple asymptotic behavior of the bounding serial time on performance as the serial time changes as the number of processors and the problem size scales upwards.

A second group of models look at execution on a different, more descriptive level. Chamberlain and Peterson [28] for example, model runtime performance as

$$
\mathrm{R}_{\mathrm{p}}=\mathrm{I}^{*}\left(\mathrm{t}_{\mathrm{comp}}+\mathrm{t}_{\mathrm{comm}}+\mathrm{t}_{\mathrm{ovrhd}}+\mathrm{t}_{\text {sync }}\right)
$$

dividing the concepts of serial and parallel times into measurable concepts that start to describe the behavior of the system in more detail. This model is iterative, looking at each wave of calculations instead of the entire problem. Zhang and Yan [4] use a similar approach, looking at the runtime of each segment "c" of the program. Zhang and Yan look at CPU time, owner utilization and use queuing methods to determine the runtime of each segment. This group of run time models also includes those derived using queuing theory and mean value analysis (MVA). Several examples using these methods were described in 1.6 .

\subsubsection{Speed}

Speed is the average number of operations performed in unit time during program execution. This is a general definition extrapolated from Zhang and Yan [4,5], who 
describe speed as the "average number of operations of different types per second for executing a program." In [33] Zhang et al. define speed as the problem size divided by the number of processors times the execution time.

\subsubsection{Speedup}

In parallel systems, speedup is a ratio of the execution time of a parallel program to a serial implementation. Most commonly speedup is simply defined as the execution time for a program on a single processor, generally the fastest in the network, over the execution time for the program in parallel over the network. Zhang and Yan [4], Mechosa et al. [34], Donaldson et al. [18], Eager et al. [17], and Kumar and Gupta [35] are examples of those who use this straight-forward definition.

Gustafson [27] and Wilson [36] both apply Amdahl's Law to speedup to get a form seen previously.

$$
\text { Speedup }=\frac{T_{S}+T_{P}}{T_{S}+\left(\frac{T_{P}}{N}\right)}
$$

where $T_{S}$ is the serial portion of the program, executed on a single processor, and $T_{P}$ is the parallel portion of the program, also as executed on a single processor. $N$, of course, is the number of processors. In this configuration, a speedup less than 1 would mean that the parallel version of the program is slower than the serial version. Wilson uses a normalized version of this expression where the numerator equals one to show maximum speedup. 
There are other ways of measuring speedup based on architecture and program size. Sun and Rover [13] assert that there are three "notions" of speedup, fixed size, fixed time, and memory bounded. Fixed size speedup is the situation described by Amdahl. This assumes that the problem being solved is of a constant size and the reciprocal of the serial fraction of execution time is the upper bound on speedup. A fixed time measure of speedup assumes an arbitrarily large problem and emphasizes the amount of work which can be accomplished over time. The speedup here would be the amount of work completed by the parallel algorithm divided by the amount of work completed by a serial algorithm. Memory bounded speedup asserts that the speedup is bounded by the amount of storage available and that memory size will increase linearly with the number of processors. In this thesis, the term speedup will reflect the fixed size speedup described above.

\subsubsection{Efficiency}

Efficiency is a measure of the amount of time a processor spends performing useful operations versus the time it spends idle during an iteration of a program. To calculate efficiency, the total program time should be taken into consideration and this number would be an average over all iterations. Gupta and Kumar [37] define efficiency as the ratio of speedup to the number of processors. This popular definition is also used by Sun and Rover [13], Eager et al. [17], Grama et al. [32], and Wilson [36]. 
Zhang and Yan $[4,5]$ have proposed an efficiency measure for a NOW platform which is based on their idea of power weight. The power weight, $\mathrm{W}$, basically compares a computer in a network to the fastest computer in that network. Zhang and Yan define efficiency by

$$
E=\frac{\sum\left(W_{j} * \frac{\left|A_{j}\right|}{S_{j}}\right)}{\sum\left(T(A, H N)-T_{\text {owner }} * W_{j}\right)}
$$

where $\mathrm{T}(\mathrm{A}, \mathrm{HN})$ is the total execution time for the network of workstations, $|\mathrm{A}|$ is a measure of problem size, $\mathrm{T}_{\text {owner }}$ is the time the processor is unavailable for parallel work, and $S$ is speed, defined as the ratio of problem size to the number of processors times the execution time.

\subsubsection{Scalability}

Scalability is a measure of how the performance of a system changes in relation to problem size and the number of processors. Scalability is the topic of much of the literature on parallel computing, though there is no one accepted definition for it as with speedup and efficiency. One notable example is the work of Grama et al. [32]. They define a scaleable system as one where the efficiency can be held constant as the number of processors increase by increasing the problem size as well. Grama et al. go on to define a metric called the isoefficiency constant to describe how the problem size must grow based on the overhead costs. 
Gustafson $[37,38]$ uses a related notion of scalability stating that the "problem size scales with the number of processors." He ultimately suggests that speedup, one measure of system performance, be measured using this scaling of problem size with the number of processors to avoid unnecessarily harsh limitations Amdahl's law would apply from fixed problem size speedup measurements. Driscoll and Daasch [29] extend Gustafson's relaxation of the bounds imposed by Amdahl's law on parallel processing by looking at the effects of problem size and the number of processors on the parallel and serial portions of the program. That is

$$
T(w, p)=\operatorname{Seq}(w, p)+\frac{\operatorname{Par}(w, p)}{P}
$$

where $w$ reflects problem size, and $p$ is the number of processors.

Zhang and Yan $[4,5]$ offer a new way of measuring scalability using their latency metric. Basically, they define scalability between two systems of differing problem size and number of processors as the ratio of the latency metric for the original system over the latency metric for the scaled up system. The latency metric was introduced in section 1.6.1.3.

Leutenegger and Sun [11] look at the advantages of using a problem scaleable system verses a fixed problem size one. They find that their task ratio, which relates the demand of a parallel task to that of other system and owner tasks on a workstation, remains constant in a scaled problem system, but declines in a fixed problem system. The higher this task ratio, the closer to the maximum possible speedup can occur. 
Sun and Rover [13] concur that scalability describes how the problem size and the number of processors, or system size, affects the performance of a particular program, or algorithm, on a particular system. They go on to suggest that this notion of scalability can be used to predict how large problem will perform on large systems by looking at how smaller problems perform on small systems. Unlike most work, Sun and Rover express scalability in an actual expression

$$
\psi\left(N, N^{\prime}\right)=\frac{N^{\prime} W}{N W^{\prime}}=\frac{T_{N}}{T_{N^{\prime}}}
$$

where $N$ and $W$ are the size of the original system and problem, respectively, and $N^{\prime}$ and $W^{\prime}$ are the size of the system and problem in the scaled up system.

\subsubsection{Concurrency}

Concurrency describes the number of tasks that can be executed at the same time in a program. Gupta and Kumar [37] denote this measurement $\mathrm{C}(\mathrm{W})$ and use it as follows. If there are $\mathrm{N}^{2}$ tasks in a problem $\mathrm{W}$, with no dependencies, then the concurrency for that problem, or program is $\mathrm{C}(\mathrm{W})=\mathrm{N}^{2}$. Zhang and Yan [4] define a similar term they refer to as degree of parallelism. The degree of parallelism for a program or algorithm is the total computing (cpu) time divided by the total execution time of the program.

\subsubsection{Granularity}

The concept of granularity is not generally discussed in the literature, except to point out that coarse-grained algorithms tend to be better candidates for parallel processing as 
they reduce communications overhead. In this context a course-grained event may require millions of operations to complete while a fine-grained process may do a very few operations [36]. Strumpen [1] has acknowledged that task granularity has a direct effect on the efficiency of the program computation, and that there is some optimal granularity to achieve good performance. Cap and Strumpen [9] go on to develop a system that changes task size with the actual load situation in their dynamic load balancing technique, though they do not directly model granularity.

\subsubsection{Selecting Metrics and Parameters}

Now that the literature has been discussed, the parameters which are necessary in the model can be selected. Also those metrics would provide meaningful information about the model's performance, and thus a system's performance, can be chosen.

Often there is a wish to know how fast a problem of a certain size will be done given a certain number of processors, or how many processors are needed to complete a certain problem in a certain amount of time. Therefore, the number of processors and the problem size must, obviously, be used in the model. Overhead is another useful metric, since overhead is a known limiting agent on distributed computing. It is necessary to know when the overhead obliterates the gain provided by using multiple processors so that processor time is used more efficiently. Since only a part of the processors' time is used, the system usage must be known. Multiplying CPU execution time by the system usage gives how long it will take when the machines are servicing other jobs as well. 
The obvious choices for performance metrics are run time, scalability and granularity. Run time is the main concern of distributed programming; the goal is to get the most amount of work done in the least amount of time. Related to this idea is scalability; knowing how big a problem, or how many processors can be used, before resources are wasted. Since the affects of granularity on scalability are of main interest in this thesis, granularity becomes more of a parameter than a metric for in this work. Efficiency is examined because it provides a way to see where resources are being wasted. Much of the literature surrounding distributed computing looks to speedup for a general performance measure of a system. Therefore, speedup will also be studied so that this work can be compared to earlier work. Speed and concurrency are mostly redundant in light of the other metrics and would provide little if any additional information about the system, so these will not be dealt with.

\subsection{Summary}

This section has given a brief overview of the ideas and theories used in this thesis. The basic purpose of this thesis is twofold. First, the work by Schaefer [3] is extended and improved to cover multiple job pools and to extend the analysis of system performance. Second and more importantly, the interaction of scalability and granularity is examined to determine if altering the granularity of the job pools can alter a systems scalability. This improvement in scalability should improve a program's feasibility for distributed processing and its performance in terms of execution time. 


\section{Chapter 2}

\section{Theoretical Basis}

\subsection{The Job Pool Paradigm}

In [3] Schaefer introduced a coordination framework to organize a given problem into a parallel format for distributed processing on a loosely-coupled NOW. The basic job pool paradigm Schaefer described is used in this thesis as a basis for the theory, model, and program structure found herein. Structural, transformational and operational primitives make up the framework of the job pool paradigm [3]. In this thesis, the paradigm described by Schaefer [3] is extended into the MPMW paradigm to cover multiple job pools and migrating workers.

\subsubsection{Structural Primitives}

The job pools and their associated workers are the structural primitives of the framework. A job pool is a structure where the tasks from a parallel job wait to be processed. A task might be placed in a job pool when a parallel job is initially split up or after the computational work on that task has finished. A worker is a process that removes one or more tasks from the job pool, processes it, and either returns it to the initial job pool or places it in the next job pool in the system. 
2.1.1.1. Job Pool Primitives

There are three possible job pool primitives; the initial job pool, the intermediate job pool, and the done pool. When a job is initially split up into tasks all the tasks ale placed in the same initial job pool. The initial job pool has no arrivals during execution time, but has departing tasks until the pool is empty. The intermediate job pool receives tasks from upstream workers serving the previous job pool and holds those tasks until the workers serving it are ready to process them. The done pool has no departures but simply collects the finished tasks from the job pool previous to it. In this thesis, a two job pool system is examined; a system containing an initial job pool a single intermediate job pool and a done pool. Figure 2-1 gives the equivalent system as an example.

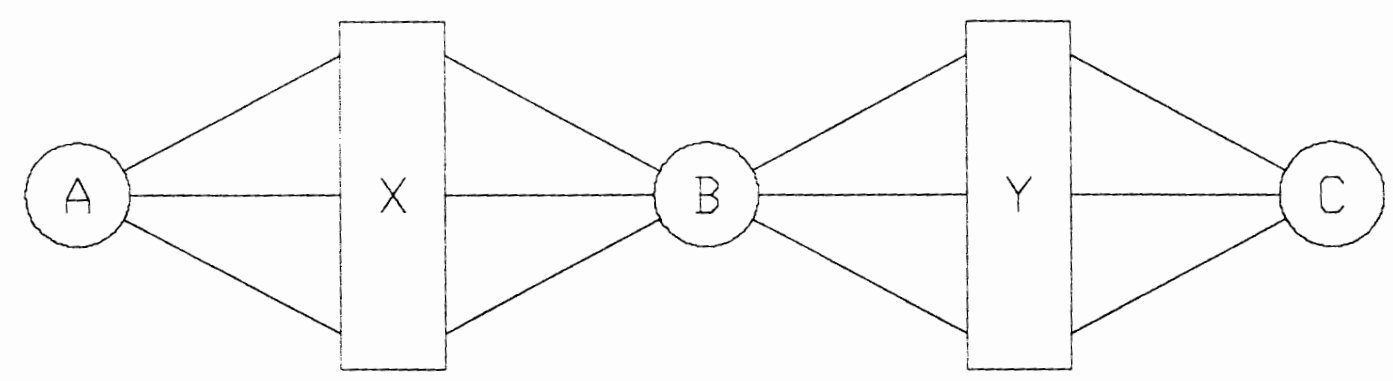

Figure 2-1. Basic Job Pool Setup

Job pool A, the initial pool, is the starting point of the system, which has a shared memory file system. The initial job is split up, by the master program, into tasks which are placed into A pool. The block marked $\mathrm{X}$ represents a number of workers that access job pool A for tasks (i.e. files). When those workers finish their work on the tasks from A, they place them into job pool B. Job pool B, the intermediate pool, is not 


\subsubsection{Job Pool Primitives}

There are three possible job pool primitives; the initial job pool, the intermediate job pool, and the done pool. When a job is initially split up into tasks all the tasks are placed in the same initial job pool. The initial job pool has no arrivals during execution time, but has departing tasks until the pool is empty. The intermediate job pool receives tasks from upstream workers serving the previous job pool and holds those tasks until the workers serving it are ready to process them. The done pool has no departures but simply collects the finished tasks from the job pool previous to it. In this thesis, a two job pool system is examined; a system containing an initial job pool a single intermediate job pool and a done pool. Figure 2-1 gives the equivalent system as an example.

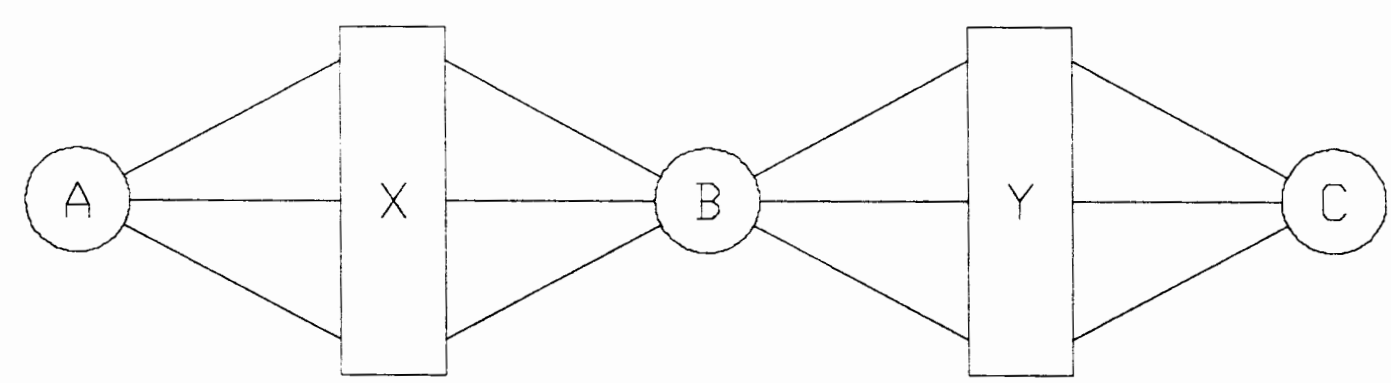

Figure 2-1. Basic Job Pool Setup

Job pool A, the initial pool, is the starting point of the system, which has a shared memory file system. The initial job is split up, by the master program, into tasks which are placed into A pool. The block marked X represents a number of workers that access job pool A for tasks (i.e. files). When those workers finish their work on the tasks from A, they place them into job pool B. Job pool B, the intermediate pool, is not 
only a repository for the $\mathrm{X}$ workers but is also a source for the $\mathrm{Y}$ workers. The workers represented by Y retrieve tasks from B, execute them, and place the "finished" product into a done pool $\mathrm{C}$. The done pool, $\mathrm{C}$, is not a job pool, but a place to store tasks after they have been through both stages of the system. No synchronization should be implied by the $\mathrm{X}$ and $\mathrm{Y}$ blocks, these blocks simply represent the workers in the system.

\subsubsection{Worker Primitives}

This system design also includes migrating workers, that is $\mathrm{Y}$ workers can become $\mathrm{X}$ workers if job pool B becomes starved for tasks. $\mathrm{X}$ workers can also become $\mathrm{Y}$ workers if job pool B becomes glutted with tasks. Starvation occurs when there are not enough tasks in the job pool for its workers to retrieve and work on. Gluttony, on the other hand, occurs when the job pool overflows with tasks. Gluttony can occur in either A or B but detrimental starvation only occurs in job pool B. Job pool A starts out with the maximum number of tasks (i.e. glutteal), it will have nothing to feed it; thus starvation in A just indicates that the first part of the work is almost done. Starvation in B, however, occurs when its workers try to retrieve more tasks than the $\mathrm{X}$ workers are placing into it. In this case, some of the $\mathrm{Y}$ workers need to migrate and become $\mathrm{X}$ workers to increase the task flow into B. At some point those workers may migrate back to the B job pool. 


\subsubsection{Job Pool Management}

Job pool management is actually a misnomer, the job pool is just a passive data structure in shared memory while the workers form the active components of the system. The job pool is a directory containing the job tasks as individual files. The worker is a spawn program created with Parallel Virtual Machine (PVM) [40] that collects tasks files from the job pool directory. Thus the worker is actually responsible for the job pool management. When tasks are said to be bundled in the job pool for the workers, the worker actually just retrieves the number of task files as dictated by the bundle size. The number of tasks per bundle determines the granularity of the pool. The worker executes the tasks and places the resulting task files into the directory of the next job pool. Once the worker executes all the tasks from the bundle, it goes to its supply job pool to get another bundle.

The worker must have code for retrieving some number of files from the supply job pool, code for working on those task files, and code to place the resultant files into the receiving directory.

To have migrating workers, each worker must have the code necessary for serving both job pools A and B. A flag in each worker will tell it which job pool it belongs to at any given time. To decide when and if a worker should migrate, the task totals from both job pools and the terminating pool are needed. The job pools and the termination pool keep track of the number of tasks they contain, via one specialized number file in each 
directory. When a worker accesses a pool to retrieve tasks it looks in this special number file, subtracts the number of tasks it took from the task number, and takes a copy of the resulting task number. When the worker places the completed task into the subsequent pool, it looks at that pools number file, adds it's tasks to the task count, and records that task count. The task counts from the workers initial and subsequent pools allow the worker to decide which job pool it should access for the next set of tasks. Two system constants reflect what is considered "starvation" and what is considered "gluttony." The constants are given as percentages and are used by workers deciding which job pool to visit. If the worker is accessing the A job pool, then using the task counts from A and B it calculates

$$
\text { job_ratio }=\frac{A}{A+B}
$$

This ratio is only a heuristic measure, it is not meant to provide analytical data. The job_ratio is compared to the starvation and gluttony constants (for example $30 \%$ and $70 \%$, respectively). If the A job pool has less than $30 \%$ of the jobs in the system, then the worker reassigns itself to the B job pool. If the A job pool has more than $70 \%$ of the jobs in the system, the worker stays with the A job pool as it is in gluttony. Similarly, if a worker is servicing the B job pool, then it computes the number of tasks in the A job pool by subtracting the tasks in the B job pool and the jobs in the terminal $\mathrm{C}$ pool from the total jobs. The ratio of $\mathrm{B} /(\mathrm{A}+\mathrm{B})$ is then calculated and comparison is done as mentioned above. If the B job pool has $30 \%$ or less of the jobs in the system, it is in starvation and the worker will reassign itself to job pool A. This increases the flow of 
jobs to $\mathrm{B}$, alleviating the starvation situation. If $\mathrm{B}$ has $70 \%$ or more of the jobs then the worker stays with B.

\subsubsection{Transformational Primitives}

A transformational primitive describes the actions a worker can take with a bundle of tasks after getting it from the source job pool and before placing it in the destination job pool. The primitives can be composed of a fork and/or a join. The concepts of a fork

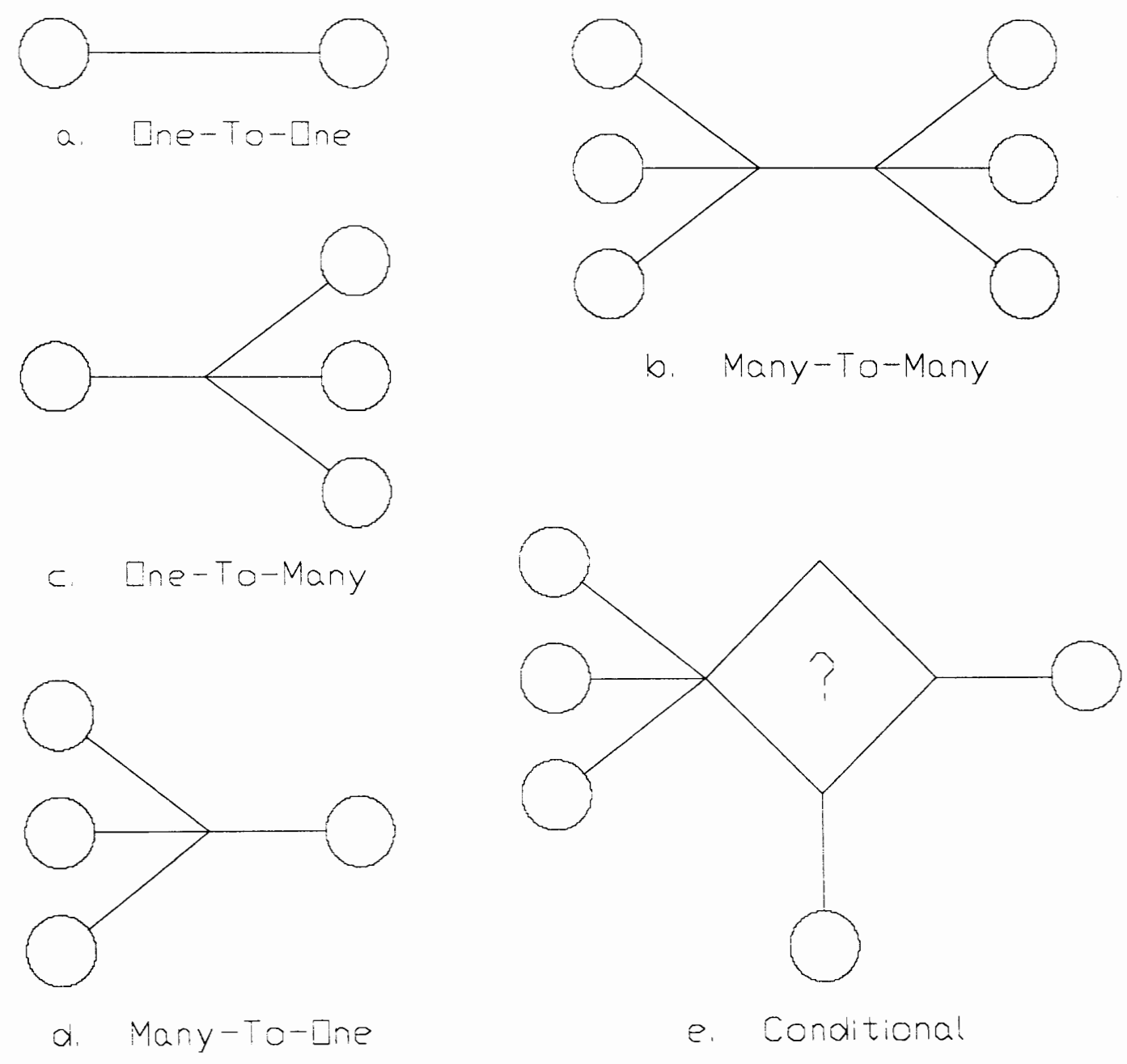

Figure 2-2. Transformational Primitives 
and a join are discussed by Bacelli and Lui $[21,22]$ and discussed in section 1.4. All the primitives described here are illustrated in Figure 2-2. These primitives were described by Schaefer in [3] and are extended here to bundles.

The simplest transformational primitive is the one-to-one primitive, where the worker collects or consumes a single bundle, does some operation on the tasks in that bundle and returns or spawns a single bundle to the destination pool. If there were only one task per bundles then one task is consumed and one task is spawned in the process. Therefore, there is a one to one correspondence between the tasks in a bundle from thesource job pool and the tasks in the corresponding bundle to the destination job pool. Other than computation no transformation takes place in this case.

The next basic transformational primitives are the one-to-many and the many-to-one primitives. In the one-to-many transformation a worker consumes a task from a source pool and performs a fork, spawning multiple tasks to the destination job pool. Conversely, in the many-to-one primitive the worker takes a number of tasks from the source job pool and performing a join, depositing a single task into the destination pool. Since this work deals with bundles, these primitives must be examined on that level as well. A one-to-many primitive represents a situation where a bundle with $x$ tasks is taken from the source pool and the corresponding bundle for the destination pool has $y$ tasks, where $y>x$ by some multiple depending on the fork of each task. The many-to- 
one primitive is just the opposite, $x>y$, as there are more tasks in the initial bundle than the final one.

There are two other primitives that combine the basic primitives to form more complex actions. The many-to-many primitive describes a worker consuming multiple tasks from the source job pool and, after computation, spawning multiple tasks to the destination job pool. The number consumed need not equal the number spawned. In terms of bundles of tasks, the many-to-many primitive involves a worker taking several bundles, doing a computation with one task (or more) from each bundle, spawning multiple tasks to be placed in separate bundles. For example, if each bundle had a single task, a worker might retrieve 5 bundles (tasks) do a calculation using all five tasks, then from the results spawn eight tasks into four bundles ( 2 tasks per bundle) into the next job pool.

The other complex transformational primitive is the conditional primitive. The conditional primitive may consume one or more tasks from the source pool and, depending upon the outcome of the computation of those tasks, may spawn one or more tasks to the destination pool. The outcome from the calculation could even effect how many tasks the worker consumes from the source on its next round of computation.

A final transformational primitive describes the action a worker performs on itself, after it spawns its current tasks to the destination job pool. This thesis deals with multiple source pools and, therefore, migrating workers, as mentioned in section 2.1.1.2. The 
worker transformational primitive allows the worker from pool A to transform into a worker for pool B. If a worker from job pool A returns to job pool A for its next tasks, no transformation occurs. If, however, that worker goes on to service job pool B next, this primitive is again needed.

\subsubsection{Operational Primitives}

The operational primitives allow the transformational primitives to occur and monitor their progress. Five operational primitives are defined to handle task management, control, and worker management.

The get_bundle and put_bundle operational primitives control task management. The get_bundle primitive consumes a bundle of tasks from the source pool and the put_bundle spawns the resulting bundle of tasks into the destination pool. Bundle size from the source pool and to the destination pool is controlled internally by the worker, but these primitives carry out the actual task transfer.

The get_lock and remove_lock primitives provide control of a job pool by a worker. Only one worker gets a particular task or bundle from a job pool, thus a worker locks the job pool it is servicing while it is getting or putting tasks in the pool. This way, multiple workers do not do their computations on the same task. Thus the locking mechanism allows us control over worker access to the pools. The remove_lock primitive destroys the lock so that other workers can proceed. 
The choose_pool operational primitive supports the worker transformational primitive. The choose_pool primitive makes the decision of which pool the worker will service next. If the same pool is chosen, then no transformational primitive is needed; however choosing the other pool invokes the worker transformational primitive.

\subsection{Model Derivation}

\subsubsection{System Variables}

The variable are defined here as a handy reference for the rest of the model description.

b = Average \# of Bundles per Processor * Cycle

$\mathrm{G}=$ Task per Bundle, Granularity of the Job Pool

$\mathrm{C}=$ Completions

$\mathrm{N} \quad=$ Number of Processors

$\mathrm{W}=$ Problem Size or work done

$I(N, t)=$ Number of workers waiting for access to the job pool

$\mathrm{U}_{\mathrm{S}} \quad=$ System Utilization

$\mathrm{Ne} \quad=$ Number of Tasks per Cycle

te $\quad=$ Time to Process Task, in a Cycle

$\mathrm{K} \quad=$ Number of Cycles

$R_{t} \quad=$ Total Response Time of the Center

$\mathrm{T} \quad=$ Total Run Time

B = Accumulated Resource Busy Time 
$D_{s}(N)=$ Service Residence Time for Service Center

$\mathrm{S}_{\mathrm{s}}(\mathrm{N})=$ Average Service Time

$\mathrm{V}_{s}(\mathrm{~N})=$ Average Number of Visits Before Obtaining Access

$\mathrm{Q}(\mathrm{N})=$ Average Number of Workers Attempting Job Pool Access

$\mathrm{T}_{\mathrm{s}} \quad=$ Time to Obtain a Single Bundle

$\mathrm{T}_{\mathrm{p}} \quad=$ Time to Compute a Single Bundle

$\mathrm{X}=$ Throughput of the Job Pool

\subsubsection{Model Derivation}

The model presented here, the Multiple Pool Migrating Worker or MPMW model, is derived for two job pools but can be easily expanded to any number of pools. The job pools are generally denoted A and B for the upstream and downstream pools, respectively. Many steps in the model's derivation are similar for both pools, thus only one pool's derivations will be presented except where the derivations differ. Major results will be presented for both pools and overall system results will include both pools. After determining expressions for runtime, an analysis of scalability, efficiency, and job pool granularity will ensue.

The MPMW model is two-tiered, there are variables and statements relating to the individual job pools and to the entire system. The system, as defined in 2.1, includes all workers, two job pools, and a terminating pool. The system can be under many possible conditions or states, but it is useful to define some special cases. One possible condition 
is that the two pools can be functionally equal, that is they have the same amount and type of work, the same average number of workers, the same calculation time for the work and the same serial time from overhead. This equivalent pools condition is defined by identical values for $\mathrm{Ne}$, te, $\mathrm{T}_{s}, \mathrm{~T}_{\mathrm{p}}$, and $\mathrm{U}_{s}$. The values for $\mathrm{Q}(\mathrm{N}-1)$ in each of the equivalent pools depends on the number of workers in the system and may be identical. Another broad case occurs when one pool dominates the other. This condition of domination is very likely as one pool will set the throughput of the system. This model seeks to alleviate the effects of bottlenecks at a job pool using the migrating workers. In extreme cases, to be used in limit analysis, the major pool will set the performance, with little influence by the minor pool.

\subsubsection{Defining Work}

In a single job pool system the amount of work done is straight forward but in multiple job pools the total work done in the system equals the work done in all the job pools, though the work type may differ among the job pools. In the MPMW model, there are two job pools, $A$ and $B$, thus $W=W_{A}+W_{B}$. The Gantt chart in Figure 2-3 charts the average behavior of one job pool, and allows definition of the work done in that job pool as

$$
W_{A}=\int\left(N-I_{A}(N, t)\right) d t
$$

where $\mathrm{N}_{\mathrm{A}}$ is the number of workers servicing the A job pool (3). Using MVA [24], averages can be used in place of distributions over the long term so $I\left(N_{A}, t\right)$ could be 


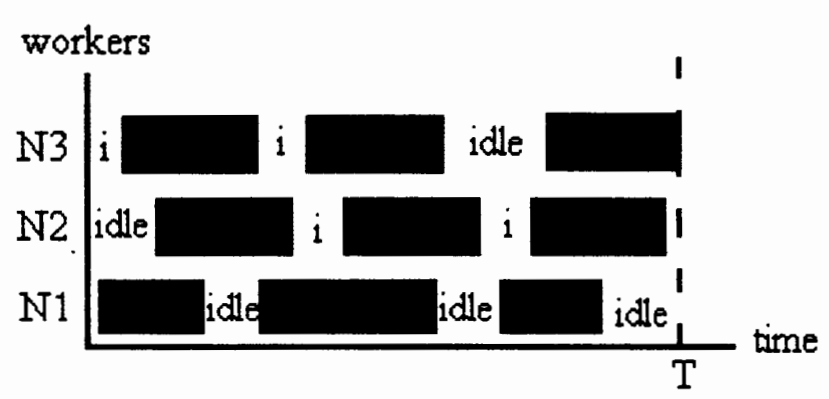

Figure 2-3. Gantt Chart

replaced with the average number of idle workers, $\mathrm{I}\left(\mathrm{N}_{\mathrm{A}}\right)$. Also, since workers may migrate from one job pool to another, $\mathrm{N}_{\mathrm{A}}$ and $\mathrm{N}_{\mathrm{B}}$ actually reflect the average number of workers servicing a job pool over a long period of time, with the total number of workers in the system being $N$. Replacing $I\left(N_{A}, t\right)$ with $I\left(N_{A}\right)$ and evaluating the integral from 0 to $\mathrm{T}$ gives a useful term for the work in a job pool

$$
W_{A}=\left(N-I_{A}(N)\right) * T
$$

The work for pool B is similarly derived

$$
W_{B}=\left(N-I_{B}(N)\right) * T
$$

but more generally

$$
W=(N-I(N)) T
$$

The total work can also be defined without the Gantt chart. The work can be measured as the amount of time necessary to complete all the tasks in a job. In a distributed system this time includes a serial fraction and a parallel fraction, $T_{s}$ and $T_{p}$, respectively. These times are related to the time to do some number of tasks in parallel. If the total number of tasks is greater than the total number of processors, then $T_{s}$ and $T_{p}$ must be 
summed over the number of iteration or cycles of the distributed program needed to complete all tasks in a job. Assuming average times for $T_{s}$ and $T_{p}$, the work is then easily calculated by

$$
W=K\left(T_{p}+T_{s}\right)
$$

where $\mathrm{K}$ is the number of cycles needed to complete the job. $\mathrm{K}$ can be calculated from the total number of tasks, the number of processors, and the bundle size or granularity. Simply stated, $\mathrm{K}$ is the total number of tasks divided by the tasks per bundle times the number of processors. For example, a program with a granularity of 2 and 10 processors processing a job with 1000 tasks will need 50 cycles to complete.

\subsubsection{Total Run Time with a Single Job Pool}

Now with an expression for the work in the system, an expression for the total run time needed to finish that work is needed. To this end, (4) is rearranged, as in Schaefer's work [3], to solve for total run time

$$
T=\frac{W}{N-I(N)}
$$

W has already been defined in several ways in section 2.2.2.1 and the number of processors, $\mathrm{N}$, is known. The only unknown is $\mathrm{I}(\mathrm{N})$.

$\mathrm{I}(\mathrm{N})$ is the number of workers waiting to access the job pool, it represents the idle time as shown in the Gantt chart in Schaefer's work. The number of waiting or idle workers 
at a job pool equals the difference between the average number of workers attempting to access the job pool, $\mathrm{Q}(\mathrm{N})$, and the average number of workers the job pool serves, $\mathrm{U}(\mathrm{N})$.

$$
I(N)=Q(N)-U(N)
$$

Combining equations (5) and (7) yields a general expression for $\mathrm{T}$ for a single job pool from [3]

$$
T=\frac{K\left(T_{p}+T_{s}\right)}{N-I(N)}
$$

\subsubsection{Total Run Time with Multiple Job Pools}

To expand (8) to represent multiple job pools, how the two job pools work together must be examined. A cycle is a unit of measure denoting when each worker in the system has retrieved a task (or set of tasks), done the tasks, and placed the tasks into the subsequent pool. During the first cycle of work all workers service the first job pool exclusively, then the workers migrate as discussed in Section 2.1. At this point both job pools are behaving like the single pool examined in 2.3.2.1, though the second pool has an incoming task stream rather then a static set of tasks. During the last cycle of operation in a distributed program, all workers are either exiting or servicing the second pool one final time. Thus the two job pools see approximately the same amount of activity, with the first pool having an extra cycle at the start of the program and the second pool having an extra cycle at the end of the program. 
Because of this overlapping of job pools, the measurements associated with each pool are not additive. For example, the $T_{s}$ for the system does not equal the $T_{s}$ of the first and second pools added together. As shown with the black "busy" boxes in the Gantt chart in Figure 2.3, $\left(\mathrm{T}_{\mathrm{sA}}+\mathrm{T}_{\mathrm{pA}}\right)$ can occur during the same time period as $\left(\mathrm{T}_{\mathrm{sB}}+\mathrm{T}_{\mathrm{pB}}\right)$. Thus the work, $\mathrm{W}$, does not change for multiple systems, it is simply understood that $T_{s}$ is the maximum of $T_{s A}$ and $T_{s B}$ and that $T_{p}$ is the maximum of $T_{p A}$ and $T_{p B}$. The addition of these two maximum values should give a worst case work for the model.

Finding the number of idle workers in a system with multiple job pools is not quite the same as using the maximum term. Since workers migrate, a continually varying number of workers service each job pool. Except for the first cycle of the programs execution, there is no way of knowing how many of the workers are at each pool. The last cycle of execution is not useful, as workers have either quit or are servicing the last pool.

During the first cycle of the program all the workers serve the first pool, thus the first cycle of execution is examined to find an expression for $\mathrm{I}(\mathrm{N})$. The number of idle workers during this first cycle will actually be an example of a worst case since there is only one pool to serve and all workers are starting at the same time. Since all workers start at approximately the same time, they all tend to attempt access to the job pool at the same time. They are more likely to have to sit idle waiting for a short period for their turn to get work. There can be other cases where one pool or the other has all the workers serving it at one time. Therefore when calculating $\mathrm{I}(\mathrm{N})$ for equation (8) in a 
two job pool system, the maximum of $I_{A}(N)$ and $I_{B}(N)$ is used. Though essentially the same technique used for $T_{s}$ and $T_{p}$ is used, the reasoning is more complex.

At this point the model still looks the same as in equation (8), but the variables have slightly different interpretations. While this expression is a good place to start, the terms $T_{s}, T_{p}$ and $I(N)$ need further investigation. $I(N)$ is explored by looking at equation (7) and the job pool throughputs and response times.

The throughput is best calculated per job pool since each job pool may have different work and granularity. This presents no difficulty, since $Q(N)$ should be described on a per job pool basis anyway. The throughput of a job pool is the ratio of the number of workers in the system over the response time of each job pool center.

$$
X_{A}(N)=\frac{N}{R_{A}(N)} \quad X_{B}(N)=\frac{N}{R_{B^{\prime}}(N)}
$$

The total response time is a function of the parallel time, $T_{p}$, and the serial time, $T_{s}$. Since the serial time is how long a worker must wait before being able to access the job pool, the total response time must reflect the service time of the job pool center. Each worker must wait for the other workers before it can access the job pool for tasks. The service time includes the time it takes that worker to access the pool and the time it spent waiting for all the workers before it

$$
S_{s A}(N)=\left[1.0+Q_{A}(N-1)\right] * T_{s A}
$$


Thus the total response time of each job pool center is

$$
\begin{aligned}
& R_{A}(N)=T_{p A}+\left[1.0+Q_{A}(N-1)\right] * T_{s A} \\
& \text { and } \\
& R_{B}(N)=T_{p B}+\left[1.0+Q_{B}(N-1)\right] * T_{s B}
\end{aligned}
$$

From (11) the throughputs of the job pools can be rewritten as

$$
X_{A}(N)=\frac{N}{T_{p A}+\left[1.0+Q_{A}(N-1)\right] T_{s A}} \text { and } X_{B}(N)=\frac{N}{T_{p B}+\left[1.0+Q_{B}(N-1)\right] T_{s B}}
$$

Using the results in (12), the average number of workers waiting to access job pool A, $\mathrm{Q}_{\mathrm{A}}(\mathrm{N})$, can be expressed as the throughput of that job pool, $\mathrm{X}_{\mathrm{A}}(\mathrm{N})$, multiplied by the service time of the center (10). The result is

$$
Q_{A}(N)=R_{A}(N) * X_{A}(N)=\frac{N *\left[1.0+Q_{A}(N-1)\right] T_{s A}}{T_{p A}+\left[1.0+Q_{A}(N-1)\right] T_{s A}}
$$

An identical expression can be found for $\mathrm{Q}_{\mathrm{B}}(\mathrm{N})$ for the $\mathrm{B}$ job pool. These expressions are recursive from the worker looking ahead in the queue to every worker in front of it $(\mathrm{Q}(\mathrm{N}-1))$.

The average number of workers a job pool serves, $\mathrm{U}(\mathrm{N})$, is also derived from the throughput and is thus a function of granularity. Multiplying the throughput, which is a rate, by the serial service time spent in the job pool gives $U(N)$ 


$$
U_{A}(N)=T_{s A} * X_{A}(N)
$$

The results obtained in (12), (13) and (14) can be substituted into (7) and simplified to give an expanded expression for $\mathrm{I}_{2}(\mathrm{~N})$

$$
I_{A}(N)=\frac{N * T_{s A} * Q_{A}(N-1)}{T_{p A}+\left(1.0+Q_{A}(N-1)\right) * T_{s A}}
$$

A similar derivation for the $B$ job pool yields $I_{b}(N)$

$$
I_{B}(N)=\frac{N * T_{s B} * Q_{B}(N-1)}{T_{p B}+\left(1.0+Q_{B}(N-1)\right) * T_{s B}}
$$

Taking the expression for total run time from (8) and substituting the definitions of $Q$ (N) from (13) and I (N) from (15), the total run time T is rewritten as

$$
\begin{gathered}
T=\frac{K\left[T_{s}+T_{p}\right]}{N-\left[\frac{N^{*} T_{s}^{*} Q(N-1)}{T_{p}+(1.0+Q(N-1)) T_{s}}\right]} \\
\text { where: } T_{s}=\max \left(T_{s A}, T_{s B}\right) \\
T_{p}=\max \left(T_{p A}, T_{p B}\right) \\
I(N)=\max \left(I_{A}(N), I_{B}(N)\right)
\end{gathered}
$$

\subsubsection{Expanding $T_{s}$ and $T_{p}$}

Equation (17) is a useful form to describe the run time of the Multiple Pool Migrating Worker model, but it is not intuitive. $T_{s}$ and $T_{p}$ are useful variables, but what they 
represent must be studied. Also, a way to compare the results in (17) to measured results is needed.

The job pool quantity represented by $T_{p A}$ and $T_{p B}$ is straight forward, it is the actual calculation time required by the tasks. Since $T_{p}$ is calculated on a per cycle basis, and since multiple tasks are being processed at once, this parallel time variable can be expressed as the number of tasks per worker per cycle, i.e. the granularity, multiplied by the actual CPU time to do each task . A system utilization term, $U_{S}$, must be added at this point as the worker processes are sharing their CPU's with other process, so the total computation time is longer when system loads are heavier

$$
\begin{gathered}
T_{p A}=G_{A} * t e_{A} * U_{S} \\
\text { and } \\
T_{p B}=G_{B} * t e_{B} * U_{S}
\end{gathered}
$$

The serial time, $T_{s}$, represents the overhead incurred from waiting to access the job pool and from getting the tasks from that job pool once access is made. Using queuing network theory [24], a general term for the serial time is described by

$$
T_{s A}=\sum D_{s}\left(N_{A}\right)=S_{s A} * V_{s}\left(N_{A}\right)
$$

$\mathrm{V}_{\mathrm{s}}\left(\mathrm{N}_{\mathrm{A}}\right)$ is the number of times a worker must visit the job pool before it gets access.

This value can be directly measured from within the distributed program. The service 
time, $S_{s}$, however, cannot be directly measured; therefore the service time per visit to the job pool, $S_{\mathrm{sA}}$, can be further defined as [24]

$$
S_{s A}=\frac{B_{s A}}{C_{A}}=\frac{B_{s A}}{X_{A} * T}
$$

The number of completions, $\mathrm{C}$, in a single cycle is a function of granularity and the number of processors.

$$
\mathrm{C}_{\mathrm{A}}=\mathrm{G}_{\mathrm{A}} \mathrm{N}_{\mathrm{A}} \quad \text { and } \quad \mathrm{C}_{\mathrm{B}}=\mathrm{G}_{\mathrm{B}} \mathrm{N}_{\mathrm{B}}
$$

Both $B_{s}$, the resource busy time, and $C_{s}$, the number of tasks completed, are direct program measurements, fulfilling the requirements. Thus the most useful description of the serial time, for each job pool, is given by

$$
T_{s A}=\frac{B_{s A}}{C_{s A}} V_{s}\left(N_{A}\right) \quad \text { and } \quad T_{s B}=\frac{B_{s B}}{C_{s B}} V_{s}\left(N_{B}\right)
$$

\subsubsection{Expanding $Q(N-1)$}

At this point the recursive $\mathrm{Q}(\mathrm{N}-1)$ term must be expanded so that (17) can be calculated directly. $Q(N-1)$ represents the number of workers attempting job pool access from the viewpoint of the next arriving worker. This is not a quantity that can be measured therefore $\mathrm{Q}(\mathrm{N}-1)$ must be defined in terms of quantities that can be measured. Observing what $\mathrm{Q}(\mathrm{N}-1)$ represents, it can be written as

$$
Q(N-1)=N(1+p)
$$


where $p$ can be seen as the fraction of all time that the servers are all busy, that is the fraction of time that the job pool is occupied and cannot service the arriving worker. Thus $\mathrm{Q}(\mathrm{N}-1)$ can be calculated by multiplying the number of workers in the system, $N$, by the fraction of time the job pool is occupied plus one (for the arriving worker).

Unfortunately, $p$ is still not easily measurable. Therefore a way to evaluate $p$ from measurable values is needed. The answer can be found in queuing theory and, interestingly, telephony. Erlang's B formula, also known as Erlang's loss formula, was developed to look at availability in wired telephone systems almost a century ago [23]. The formula was used to give the blocking probability of incoming calls. If a call came in and all the servers were busy then the call was lost and the user had to try again. The same thing occurs within the system when a worker needs more work. If the job pool it serves is busy then that worker departs (is lost) and attempts accessing the job pool again. The alternative system is to place workers needing tasks in a queue and have them service the job pool on a first come first serve basis. This queue discipline adds to the complexity of the MPMW system.

Erlang's B formula can be used to describe the fraction of the time that all servers, or job pools are busy, and are unable to service an arriving worker. Girard [39] refers to this as the time congestion of the system. Erlang's loss formula is expressed as 


$$
\rho=\frac{\frac{\alpha^{N}}{N !}}{\sum_{k=0}^{N-1} \frac{\alpha^{k}}{k !}}
$$

where

$$
\alpha=\frac{T_{p}}{T_{s}}
$$

As shown in the previous section, $T_{p}$ and $T_{s}$ can be calculated from system measurables. Thus, Erlang's loss formula can be used to define $\mathrm{Q}(\mathrm{N}-1)$ in easily measured system variables by substituting (24) into (23).

$$
Q\left(N_{A}-1\right)=N\left(1+\frac{\frac{\alpha^{N}}{N !}}{\sum_{k=0}^{N-1} \frac{\alpha^{k}}{k !}}\right)
$$

\subsubsection{Limit Analysis of Run Time}

At this point it is useful to look at the limit behavior of the system described by (17) to assure ourselves that this model is feasible. First, what the system does when one pool dominates the other is examined, that is when, $t_{A} \gg>t e_{B}$ and $B_{A} \gg>B_{B}\left(\right.$ or $\left.V s_{A} \gg>s_{B}\right)$. In this instance, the actual task computation time for one pool is much larger than the other and the busy time (or number of visits) of the same job pool is also much greater than for the minor pool. The dominating pool, A, controls the system throughput and run time, because it is so much slower. The minor pool has a higher completion rate, though it could be limited by the dominating pool. From (18), as te $\mathrm{A}_{\mathrm{A}}$ becomes greater 
than te $\mathrm{B}_{\mathrm{B}}, \mathrm{T}_{\mathrm{pA}}$ will dominate $\mathrm{T}_{\mathrm{pB}}$. Likewise, from (26), a disparity in resource busy times or the number of visits will affect the relative values of $T_{s A}$ and $T_{s B}$. In this case, $T_{s A}$ will be the maximum value. Thus the limit as pool A dominates pool B becomes

$$
\lim _{A>B} T=\frac{T_{s A}+T_{p A}}{N-\left[\frac{N T_{s B} Q_{B}(N-1)}{T_{p B}+\left(1.0+Q_{B}(N-1)\right) T_{s B}}\right]}
$$

This is very similar to what the expression for $\mathrm{T}$ would be for a single job pool, the only difference being in that the numerator values come from the major job pool and the denominator values come from the minor job pool. The $\mathrm{I}(\mathrm{N})$ factor in the denominator is largely determined by the minor pool ratio of $T_{s B}$ to $T_{S A}+T_{s B}$.

A second case occurs when the serial times, $\mathrm{T}_{\mathrm{sA}}$ and $\mathrm{T}_{\mathrm{sB}}$, become much larger than the parallel times, $\mathrm{T}_{\mathrm{pA}}$ and $\mathrm{T}_{\mathrm{pB}}$. It is expected that the overall time, $\mathrm{T}$, to be higher as the benefits of distributed processing are lost. Looking again at the definitions of $T_{s}(19)$ and $T_{p}(18)$ notice what happens as the serial costs increase in a single pool

$$
\left(\frac{B_{s A}}{C_{A}}\right) V_{s}\left(N_{A}\right)>>G_{A} t e_{A} U s
$$

Assuming $U_{S}$ to be 1 , it can be seen that the serial time can has a large effect when the resources busy time is high, the number of visits before success is high, and the number of completions is low. These two conditions are related, the number of visits will increase if the busy time increases and the number of completions will go down as the job pool is busy more of the time, thus $B_{\mathrm{sA}}$ can be seen as an indicator of the serial time. 
The parallel expression is similarly controlled by the task time te $\mathrm{A}_{\mathrm{A}}$. Thus as $\mathrm{B}_{\mathrm{sA}}>>\mathrm{te}_{\mathrm{A}}$ and $\mathrm{B}_{\mathrm{sB}}>>\mathrm{te}_{\mathrm{B}}$, a worker spends more time attempting to get tasks than actually working on them. From this the limit of (17) as the serial time becomes larger than the parallel time is

$$
\lim _{T_{s}>T_{p}} T=\frac{T_{s A}}{N-\left[\frac{N T_{s A}}{T_{s A}+T_{p A}}\right]}
$$

Thus as $\mathrm{T}_{\mathrm{sA}}$ becomes much larger than $\mathrm{T}_{\mathrm{pA}}$ the denominator becomes very small and $\mathrm{T}_{\mathrm{sA}}$ dominates the numerator. As the denominator approaches zero, the total execution time will increase as expected when the serial time starts to rise.

The same reasoning can be used to look at the opposite extreme, when the values of $T_{p A}$ and $T_{p B}$ are larger than the serial values of pools $A$ and $B$. In this case, $t_{A}$ and te $e_{B}$ get much larger than the resource busy times, $B_{s A}$ and $B_{s B}$, indicating that the $T_{p}$ terms will dominate (17)

$$
\lim _{p}>>T_{s} T=\frac{T_{p A}}{N-\left[\frac{N}{T_{p A}}\right]}
$$

In this case, the numerator is dominated by $\mathrm{T}_{\mathrm{pA}}$ and the denominator approaches $\mathrm{N}$ as the value of $T_{p A}$ increases. This means that $N$ will be reduced less and less as $T_{P A}$ is increased, and therefore the numerator will be divided by a larger number than in (27) resulting in a run time decrease, as expected. 


\subsubsection{Amdahl's Law and Run Time}

The results in (17) can be examined in regard to the limits on parallel performance that Amdahl's law describes [27]. Amdahl's Law can be stated using the variables representations in this thesis

$$
T_{\text {Amdanl }}=K *\left(T_{S}+\left(\frac{T_{P}}{N}\right)\right)
$$

Before comparing (17) to Amdahl's law, (29) is rewritten to reflect the concepts in Driscoll and Daasch [29]

$$
T_{\text {Amdahl }}=T_{s}(W, N)+\frac{T_{p}(W, N)}{N}
$$

It can be shown that the result in (17) does fit the limiting nature of Amdahl's law and fits the form of (30). By defining the functions represented by $T_{S}(W, N)$ and $T_{p}(W, N)$ the MPMW runtime model can be neatly put into form. From (17), the numerator can be split up to yield the following functions of $T_{S}$ and $T_{P}$

$$
\begin{gathered}
T_{S}(W, N)=\frac{T_{s}}{N-\left[\frac{N T_{s} Q(N-1)}{T_{p}+(1.0+Q(N-1)) T_{s}}\right]} \\
\text { and } \\
T_{P}(W, N)=\frac{T_{p}}{1-\left[\frac{T_{s} Q(N-1)}{T_{p}+(1.0+Q(N-1)) T_{s}}\right]}
\end{gathered}
$$


Substituting the expressions in (31) into (30)

$$
\left.T=\left(\frac{T_{s}}{N-\left[\frac{N T_{s} Q(N-1)}{T_{p}+(1.0+Q(N-1)) T_{s}}\right]}\right)+\frac{T_{p}}{1-\left[\frac{T_{s} Q(N-1)}{T_{p}+(1.0+Q(N-1)) T_{s}}\right]}\right]
$$

Equation (32) is written to show its resemblance to the form in (30) with a serial portion added to a parallel portion over the number of processors. Presenting (17) like this shows another important idea, that the serial time is not independent of the number of processors or the parallel time. The total time a program spends in purely sequential mode can be influenced by the number of processors in the system and the time it takes those processors to do work in parallel. The overhead related to setting up machines and data for a parallel portion of a program, for example, can be done serially on a single processor, adding this overhead to the total serial time. The time for such overhead depends on the number of processors present; it may take longer to set up many processors verses a few.

\subsubsection{Speedup}

Speedup reflects the relative performance of systems with different numbers of processors (or workstations) [13]. Thus

$$
S p=\frac{T(1)}{T(N)}
$$


where the same job is run in both cases. Using (17) and (33), the expression for speedup would be

$$
S p=N-\left[\frac{N^{*} T_{s} Q(N-1)}{T_{p}+(1.0+Q(N-1)) T_{s}}\right]
$$

Speedup can be looked at on a per job pool basis or on an overall system basis. The speedups of individual job pools are not additive, the minimum job pool speedup determine the system speedup. Also, since the system is heterogeneic, the fastest machine should be used to determine $\mathrm{T}(1)$. Speedup is often used in the literature to determine relative performance gains (or losses) from adding processors to the system; thus it is used in this thesis.

\subsubsection{Limit Analysis of Speedup}

Again, it is useful to examine the limits of the results to see if the expressions make sense. Speedup is expected to increase as the parallel term dominates and decrease as the serial term dominates as it is the parallel computation that gives the speedup over a single processor in the first place. In the case of a dominant pool, that is a pool with much greater values for te and $\mathrm{B}_{\mathrm{s}}\left(\right.$ or $\mathrm{V}_{\mathrm{s}}$ ), the larger pool is expected to limit the speedup since it becomes the bottleneck in the system. Looking at the limit of (34) as te $e_{A} \gg>e_{B}$ and $B_{s A} \gg>B_{s B}$ and see that $T_{s A} \gg>T_{s B}$ and $T_{p A} \gg T_{p B}$. These inequalities show little difference, it is the $T_{s} /\left(T_{s}+T_{p}\right)$ ratio that controls the denominator, so if the ratios do not change, it will not matter how much larger $\mathrm{T}_{\mathrm{sA}}$ and $\mathrm{T}_{\mathrm{pA}}$ get. Thus it makes no 
sense to look at speedup as one pool or the other gets large, since it is the value of the serial and parallel times that ultimately control speedup.

$$
\lim _{A>B} S p=N-\frac{N * T_{s}^{*} Q(N-1)}{T_{p}+(1.0+Q(N-1)) T_{s}}
$$

The limit as the serial times, $T_{s A}$ and $T_{s B}$, become much greater than $T_{p A}$ and $T_{p B}$ is quite obvious in (36). When serial time dominates the speedup goes to zero. In a completely serial case, the speedup would be zero.

$$
\lim _{T_{s}>T_{p}} S p=(N-N)
$$

Conversely, the limit as the parallel terms, $T_{p A}$ and $T_{p B}$, dominate the serial terms, $T_{s A}$ and $T_{\mathrm{sB}}$, is

$$
\lim _{p} S p=\left(N-\frac{N}{T_{p A}}\right)
$$

where the Speedup approaches $\mathrm{N}$ as the parallel term increases.

\subsubsection{Granularity}

Granularity has already been used to define the completions of a job pool, now it will be discussed formally. The granularity of a job pool reflects how many tasks a worker takes from the job pool per access. Increasing the granularity by increasing the number of tasks taken at a time reduces the benefits of parallelism, but also reduces the overhead costs. Ideally, decreasing granularity increases parallelism and overhead. A balance must be obtained between the benefits and costs to determine the optimal 
granularity. In this model granularity is given by $\mathrm{G}$ and is in term of tasks per bundle. During each visit to the job pool center, a worker gets a bundle of tasks to work on, G defines the number of tasks in that bundle. By definition granularity must be determined for each job pool. Thus in the two job pool system, there is $\mathrm{G}_{\mathrm{A}}$ and $\mathrm{G}_{\mathrm{B}}$. The only time the granularity can be talked about in general system terms is if $G_{A}=G_{B}$, otherwise calculations including granularity must be on a per job pool basis. Granularity ranges from one to the total number of tasks in the job (i.e. the serial case).

The total time to finish all A tasks for example, is affected by the granularity of the A job pool, $G_{A}$, by increasing the parallel time $T_{p A}$ and decreasing the number of times a worker must re-access the A job pool for additional work. Simple algebraic manipulation of (18) gives the expressions for $G_{A}$ and $G_{B}$

$$
G_{A}=\frac{T_{p A}}{t e_{A} U_{S}} \quad \text { and } \quad G_{B}=\frac{T_{p B}}{t e_{B} U_{S}}
$$

The serial term, $\mathrm{T}_{\mathrm{sA}}$ is also a function of granularity, substituting (21) into (22) and solving for $\mathrm{G}$ shows the relationship

$$
G_{A}=\frac{B_{A} V_{s A}}{T_{s A} N} \quad \text { and } \quad G_{B}=\frac{B_{B} V_{s B}}{T_{s B} N}
$$

Thus the $T_{s}$ decreases as granularity increases, showing the decrease in overhead since fewer trips to the job pool are required when granularity is increased. 
Assuming $T_{s A}, T_{p A}$ and $I_{A}(N)$ are dominant, the definition of $T_{p A}$ in (18) and $T_{s A}$ from (21) and (22) can be substituted into (17) to demonstrate the connection between run time and granularity

$$
T=\frac{K\left[\left(\frac{B_{A} V_{s A}}{G_{A} N}\right)+\left(G_{A} t e_{A} U_{s}\right)\right]}{N *\left[\frac{\left.B_{A} V_{s A}\right) * Q_{A}(N-1)}{G_{A} N}\right)\left[\frac{G_{A} t e_{A} U_{S}+\left(1.0+Q_{A}(N-1)\right) *\left(\frac{B_{A} V_{s A}}{G_{A} N}\right)}{\left[G_{A}\right.}\right]}
$$

From (39), the granularity affects the run time directly in the numerator and in the denominator. Increasing granularity would increase the numerator and the denominator, lowering the run time until the denominator's upper limit of $N$ is reached. At that point increasing the granularity only increases the numerator, increasing overall run time.

The effects of granularity is also seen in the calculation of the number of cycles, $\mathrm{K}$, the program runs. $K$ is calculated by looking at the total number of tasks, the number of job pools, and the number of completions in the dominant pool

$$
K=\frac{(\# \text { tasks })(\# \text { jobpools })}{C}
$$

In (10) the completions of a pool are determined by the granularity and the number of processors involved. Thus the effects of job pool granularity are inversely proportional to $\mathrm{K}$, a major multiplier in the model. 


\subsubsection{Limit Analysis of Granularity}

From the results presented in (39) the effects granularity has on run time, T, is examined by taking the limit of (39) as the job pool granularities approach the total number of tasks and as they approach 1 .

As job pool granularity approaches the total number of tasks, parallelism is lost and the process becomes serial. Thus as $\mathrm{G}_{\mathrm{A}}=\mathrm{G}_{\mathrm{B}}=$ total tasks, the iteration counter, $\mathrm{K}$, is lost and many more processors are left idle. Looking at (39), as the value of granularity increases, the numerator of (17) increases as does the denominator. The granularity has a more direct effect on the numerator, since the denominator value is limited by $\mathrm{N}$.

Thus this condition leads to greater values of $\mathrm{T}$. Thus the cost of losing parallelism by making the bundle size too large is seen. Conversely, as the job pool granularities go to one, there is an increase in $\mathrm{K}$, the multiplier of the entire runtime expression. While the base expression from (39) will decrease, multiplying it by a much higher $\mathrm{K}$ value destroys the benefit. Thus, having singular granularity, where workers get single tasks at a time, can be as detrimental as doing the job serially.

There can be cases where the granularities of the two job pools become very different, the granularity of one job pool may approach 1 while the other approaches the total task number. Since the maximum $T_{s}$ and $T_{p}$ of the system is used, the choice for granularity is indirect, depending on other system values that determine $T_{s}$ and $T_{p}$. It is possible that to use the granularity of both job pools. 


\subsubsection{Scalability}

Scalability reflects how the amount of work must increase as the number of processors increases to maintain the total execution time. It is hypothesized that changing the granularity of a job pool can improve the scalability of a system. From Sun and Rover, scalability, $\psi$, is given by

$$
\psi\left(N, N^{\prime}\right)=\frac{T_{N}}{T_{N}^{\prime}}=\frac{N^{\prime} * W}{N * W^{\prime}}
$$

Speedup is basically a special case of scalability, where the $T_{N}$ is the serial configuration, and $\mathrm{T}_{\mathrm{N}}$ ' is the scaled up configuration. A more general expression for scalability can be written, relating it to the job pool granularities from (39)

$$
\psi\left(N, N^{\prime}\right)=\frac{\frac{K\left[T_{s}+T_{p}\right]}{N-\left[\frac{N T_{s} Q_{A}(N-1)}{T_{p}+\left(1.0+Q_{A}(N-1)\right) T_{s}}\right]}}{\frac{K^{\prime}\left[T_{s}^{\prime}+T_{p}^{\prime}\right]}{N-\left[\frac{N^{\prime} T_{s}^{\prime} Q_{A}^{\prime}\left(N^{\prime}-1\right)}{T_{p}^{\prime}+\left(1.0+Q^{\prime}{ }_{A}\left(N^{\prime}-1\right)\right) T_{s}^{\prime}}\right]}}
$$

where $T_{s}$ and $T_{p}$ are both functions of granularity. Restating from (18) for clarity

$$
T_{p A}=G_{A} * t e_{A} * U_{S}
$$

shows the relationship between $T_{p}$ and G. Using both (21) and (22) the relationship between $T_{s}$ and $G$ is

$$
T_{s A}=\frac{B_{s A}}{G_{A} N} V_{s}\left(N_{A}\right)
$$


Again, this assumes that $\mathrm{T}_{\mathrm{sA}}$ and $\mathrm{T}_{\mathrm{PA}}$ are the maximum values in the system. Equation (41) shows that the scalability of the system is a function of granularity, so one could expect changes in granularity to change the scalability of the system.

\subsubsection{Limit Analysis of Scalability}

One of the questions this work looks at is the effects of job pool granularities on the scalability of a system. The model can be examined by looking at the limit behavior as the granularities grow very large and very small.

As the granularities of the job pools approach the total number of tasks (i.e. a sequential case), a decrease in scalability is expected. Assuming that $N^{\prime}>N^{\prime}$ and $W^{\prime}>W, G^{\prime}$ has a higher potential value than G. Looking at scalabilty via (41) as both granularities increase shows that the scalability does indeed decrease, according to the model. As the granularities approach one, however, the scalability's dependence upon granularity decreases. The ratio of $\mathrm{N}^{\prime}$ to $\mathrm{N}^{\prime}$ and $\mathrm{W}$ to $\mathrm{W}^{\prime}$ affects the scalability more than having the granularities of both pools near one.

\subsubsection{Efficiency}

The efficiency of the system may also be examined and related to the granularity of the job pools. Efficiency can be expressed as the speedup of the system divided by $\mathrm{N}$ [13]. Thus from (34) and (38) efficiency and granularity are related by

$$
E=\frac{S p}{N}=\frac{\left(N-\left[\frac{C T_{s} * Q(N-1)}{T_{p}+(1.0+Q(N-1)) T_{s}}\right]\right)}{N}
$$




\subsubsection{Limit Analysis of Efficiency}

The limit behavior of (42) will be nearly identical to that of the speedup expression (34). The results from section 2.2.4.2 are just divided by $\mathrm{N}$ in the denominator. As the serial term $T_{s}$ dominates, expression (42) becomes

$$
\lim _{T_{s}>T_{p}} E=\frac{(N-N)}{N}
$$

indicating that as the serial costs become large, the efficiency goes to zero, as is expected.

As $\mathrm{T}_{\mathrm{p}}$ dominates, (42) becomes

$$
\lim _{T_{p}>T_{s}} E=\frac{\left(N-\frac{N}{T_{p A}}+\frac{N}{T_{p B}}\right)}{N}
$$

and efficiency approaches one. Both efficiencies will be less than one, but the efficiency will be higher when the parallel behavior dominates than when the time for serial work increases. This outcome is expected because the parallel terms represent useful work while the serial terms represent time wasted in overhead, thus the less overhead; the more efficient the system. 


\subsubsection{Summary}

At this point the MPMW model has been developed and its limit behavior examined to see that the expected results occurred. The model was then used to look at definitions of scalability, speedup, efficiency and how they relate to granularity. The next step is to physically setup a two job pool distributed system and to determine how to make the necessary system measurements to compare with the model's description. The MPMW needs to be tested to see if the model "fits" a real life situation. The next section describes the physical programming setup and relates the system measurements to the variables used here.

\subsection{Multiple Pool Migrating Worker Implementation}

The previous chapters have described MPMW framework and model conceptually. Here it is discussed how everything will work physically and how measurements will be made for comparison to the model presented in Section 2.2. It is an ultimate goal to provide a simple, useful framework for those with few programming skills to use for distributed computation. Another goal is to present a conceptual model that explains the computation while providing useful performance information; the measurement techniques mentioned here provide that information.

\subsubsection{Physical Framework}

The physical framework is the actual interface the user would see and have to deal with. Conceptually there are job pools, workers and tasks, physically these correspond to 
directories, programs, and files. In implementation a locking mechanism must limit job pool access and log files must keep track of those tasks. The framework setup is done via simple scripts, while the program is written in C using PVM. PVM [40], Parallel Virtual Machine, is used to start a lock master and spawn workers.

A single main directory holds the program, scripts and subdirectories related to framework. As alluded to in Section 2.1, the job pool primitives are inert structures that hold tasks before and after they are consumed by workers. Thus, the job pools are implemented as subdirectories. Each pool in the system, the initial pool, the job pool, and the done pool, is a separate subdirectory containing an associated log file. The only difference between the three types of job pool directories is the contents of their log file. The initial pool $\log$ file and the done pool log file both contain the number of tasks in the pool (i.e. directory) and the name of the last task placed in that directory. The job pool directory must keep track of these quantities as well as the total number of tasks that have passed through that job pool directory.

The tasks are files that are kept in the pool directories until consumed by a worker, after which they are placed in the next directory. Each task file contains only the data that is to be worked on; it contains no code. The worker is physically a program that does all the active work in the system. Each worker program is identical; the first instance of the program spawns duplicates of itself via PVM. Each worker creates its own working subdirectory under a worker subdirectory to provide a private area for computation. 
Conceptually, the worker consumes tasks from the initial pool, delivers them to the job pool where another worker consumes it and places it in the done pool. In implementation, the worker program moves a number of tasks files (equaling a bundle) from the initial pool subdirectory to its working subdirectory, completes its prescribed computation based on data in the task files, and it moves the updated or finished files to the job pool directory. A worker program servicing the job pool then moves the tasks from the job pool directory to its working directory, completes the computation and moves the files to the done pool directory. At each stage the worker program must read in the information from the log file to determine which task files to get. After computation the program updates the destination pool directory's log file, appropriately.

Because logfiles update to keep track of tasks, only one worker program accesses a pool directory at a time. To do this a locking mechanism must be instituted to prevent multiple concurrent accesses. The locking mechanism has stringent requirements, the time to create the lock must be shorter than the time to check for that lock. This requirement prevents a second worker program from seeing an unlocked directory while the first worker program is in the process of getting a lock to that directory. After being spawned by the master program, the workers act independently for the rest of the run. After spawning, the master program becomes the lock master, controlling the locking and unlocking of the pools. Upon initiation, the lock master prepares to receive requests for information from the workers. When a worker sends a request for lock information for a particular pool the lock master returns the current value for the lock 
on that pool. If that value indicates a lock, the worker continues to request lock status until the pool is unlocked. If the lock master sends a worker a value indicating an unlocked pool, it immediately changes the value to a locked value, indicating that worker has the lock. Thus if a worker receives the indication that the pool is unlocked it assumes it has the lock on that pool. After the worker is finished at a particular pool, it sends notice to the lock master to change that pool's lock status to unlocked, then the next worker requesting information about that lock gets the lock. This continues until a worker informs the lock master it is no longer needed because the tasks have been removed. At that time the lock master exits and the run terminates.

The master program contains the spawning code and the lock master code. The worker spawn program contains the task file handling code, the worker migration code, the task computation code, and the shutdown code. All the sections except for the task computation code may remain essentially unchanged for different users and different applications. The task computation code is kept underneath its own generic function call for easy transformation to other applications. The tasks files contain the data to be used by the computation code for the required work.

The worker migration code allows the worker to decide whether to keep servicing its pool or to move or migrate to the other pool. Essentially, the worker determines the number of tasks left in each pool and finds the ratio of the tasks left in its pool to the total tasks in both pools. This ratio determines where the worker goes. If a worker's 
current pool still has the majority of the tasks the worker stays where it is at. If the other pool has a significant majority of the tasks, as set by the user, then the worker will migrate over to service that pool. Migrating to one pool or the other is done via function call; moving to the other pool is done calling the function for that pools code.

Scripts are in charge of initializing the directory structure and resetting for multiple runs. The initializing script sets up the initial, job and done pool subdirectories, the worker subdirectory and their associated log files. The resetting script clears all the directories and resets the log files to default values.

\subsubsection{System Measurements}

The are two main types of system measurements, time measurements and counters. Function calls to gettimeofday() determine the time based quantities. The time to get a lock, for example, is measured by comparing the result of gettimeofday () calls before and after the lock function. The time to release a lock is measured the same way, as is the task time. An average of these measurements are necessary for the model. Execution time is useful for the entire job is useful for evaluating the model and is measured by comparing calls to the UNIX date command at the start and end of the program. While the time given by the date command is not accurate to the millisecond, it is suitable for the purposes of this thesis and will not produce undue error.

The spinlock count, in contrast, is a variable incremented each time a worker receives a locked response in its request loop. The number of bundles and the number of 
completions for a processor are likewise counted in the appropriate loops. Every time a worker retrieves another bundle, its bundle count increments and each time it places a completed task in the subsequent job pool, the completion count is incremented.

System utilization is the only other measurement needed to use the MPMW model. System utilization reflects the percent idle time of the machine as given by the UNIX command $v$ mstat. If a processor has an idle percentage of $87 \%$, for example, then $\mathrm{U}_{\mathrm{S}}$ is 1.13. This work deals with a network of processors, therefore a system idle percentage must be found as an average of the idle times of the component processors. Since the model needs to predict the behavior of the program (including run time), an estimate of what the system idle time will be during the computation is used. For this estimate, the idle percentages of a few machines in the network can be observed for a long period of time calling vmstat every hour and the data averaged and collated by day and hour.

This method is similar to ideas presented by Mutka in [12], though a few representative machines are examined rather than all the machines for the averages. It is important that among these few machines are some of the more heavily loaded machines as well as the lightly loaded so that the average will be useful.

\subsection{Summary}

In this chapter, the theory portion of this thesis is presented. This work was based on the Job Pool Paradigm presented by Schaefer, then another pool and some functionality was added to it. From here any number of job pools can be added with only small 
predictable changes to the model and its associated performance metrics. After deriving the MPMW model, the limits were analyzed to test for feasibility. The limit behavior is indicative of the behavior expected under such circumstances. Along the same lines, the MPMW model was compared to Amdahl's Law with satisfying results.

Speedup, job pool granularity, scalability, and efficiency were introduced in terms of the model. The interaction between scalability and granularity was examined on a theoretical level. These discussions set the stage for model verification. Then the physical program framework and the associated measured values were described. These measured values either stay the same as the problem and system are scaled up or can be averaged over a series of measurements for use in the model. Now that theory involved has been discussed, three "real world applications" will be examined, one of which becomes the application used in this thesis. A set of experiments to verify the predictive MPMW model is developed. 


\section{Chapter 3}

\section{Applications}

The job pool paradigm and the MPMW model are not suitable for all applications. The characteristics that an application should have to be a successful candidate for this distributed paradigm will be examined. Three example candidate applications will be discussed including the one used in the experimentation portion of this thesis. LU decomposition with back substitution. The other two applications are potential candidates for MPMW implementation and include plasma simulation and genetic database searching.

\subsection{Characteristics for Mapping to MPMW Model}

As with all distributed computing, the grain size and the communication/computation ratio have a large influence on performance in the MPMW paradigm. Very fine grained applications that require frequent communication updates are poor candidates for this paradigm. The multiple pool, migrating worker scenario does increase the probability that a particular application could benefit from distributed computing. A small grained tasks could be grouped in a bundle, increasing the granularity of the program. The job pools and lock master potentially provide greater avenues for communication with a minimal affect on the system or performance.

Even with the advantages of the MPMW paradigm, coarse-grain, function-parallel tasks will still give the best performance since communication costs will be at a minimum. 
Function-parallel tasks could be completely independent, like running 100 different simulations at once, with no collaboration of the resulting data. Agenda parallel tasks are also appropriate, as pointed out by Schaefer [3], since an agenda of tasks could be easily split into multiple job pools with one item of the agenda per job pool. The migrating workers would be able to service any item in the agenda depending on which job pool they were at. Agenda parallel tasks could be where the same sequence of computations are done to multiple sets of data. Each data set represents a task. Schaefer [3] identifies parametric sensitivity analysis, optimization, computer aided drafting, ray tracing and database management as potential agenda parallel candidates.

As Schaefer's [3] paradigm is the basis of this MPMW paradigm, her discussion of appropriate characteristics relates directly. Some constraints necessary to insure performance with Schaefer's paradigm can be avoided with the MPMW paradigm. For example, Schaefer points out that result parallel tasks are too fined grained, but could be transformed into larger data objects, the bundling of tasks in the MPMW paradigm effectively does this transformation. Overall, the existence of migrating workers reduces the effects of the task size decision in a distributed system. If the tasks in one pool are too large, causing a bottleneck in the job pool pipe, then workers can move to assist that job pool until the crises is past, then migrate off to other pools.

There are two dimensions provided by multiple job pools, pipelining and parallel execution. A possible scenario, Figure 3-1, for a job might include three or more 
sequential job pools, a finished task from one pool is placed in the next pool for the next permutation to be done, and so on in a pipeline fashion. A split may occur at the second job pool, with the result of a compare deciding which of two job pools the resulting task is placed in. Each recipient pool has multiple subsequent pools resulting in a parallel pipeline structure, with tasks being executed in both branches simultaneously. Applications that can be decomposed into this sort of structure will be served best by the MPMW paradigm.

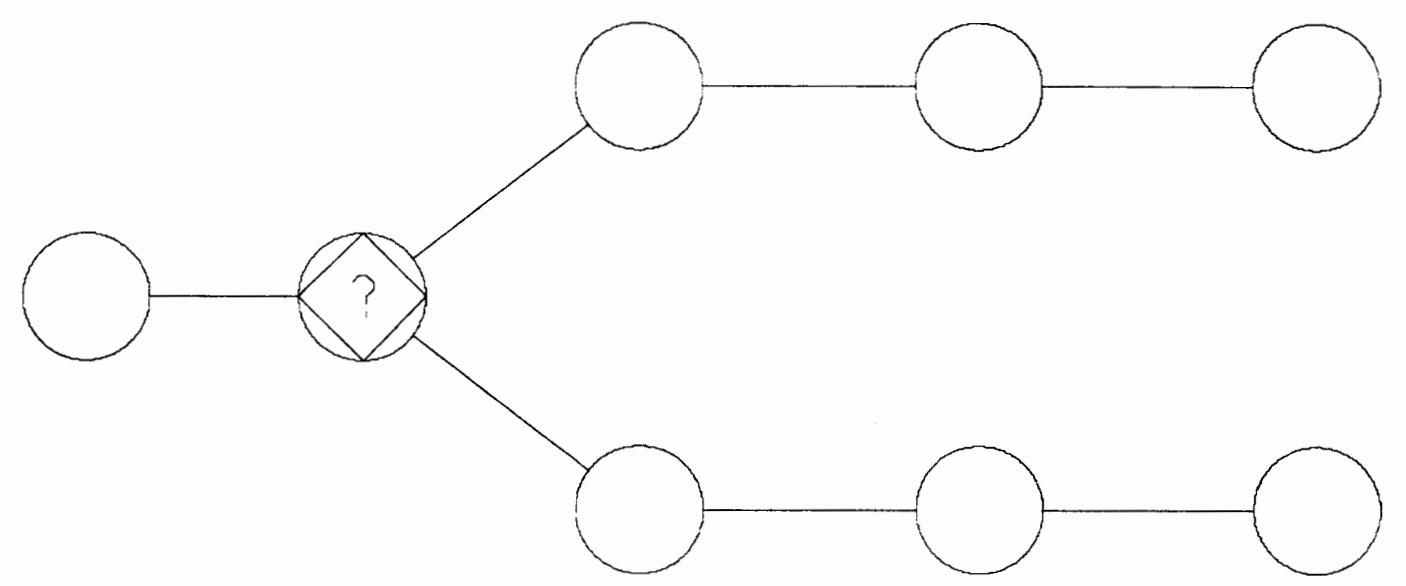

Figure 3-1. Job Pool Representation of Pipelining and Parallel Execution

While minimal communication still provides the best performance, the job pools and the lock master strive to minimize performance hits caused by needed communication. The $\log$ file in a job pool is designed to pass information about the number of tasks left in the pool to the next worker. This file could be altered to be hold other state information from one worker to another. A separate file or program could also be instituted to alter tasks as they pass through or workers when they access the pool to pass information around. The lock master keeps track of which worker has which pool locked. Every 
worker calls the lock master to request and release a lock. Like the log file the lock master could be altered to facilitate the dissemination of information. It should be noted that the lock master could become a bottleneck in the system, thus using the job pool for communication, rather than the lock master is the most reasonable procedure.

\subsection{Selected Examples}

\subsubsection{Plasma Simulation}

By definition, simulation is to "give the appearance or effect of" [41] thus scientific simulations of circuit faults, weather, or plasma are meant to give the effect (results) without undertaking the physical event. Modeling of such events is usually very complex and thus the simulations take vast amounts of processor time. Distributed computing can either reduce the time for a single simulation or at least allow the user to run multiple simulations at once, reducing the overall wall clock time needed to get results. In [2], Nibhanupudi et al. use a network of workstations to perform plasma simulations.

Plasma is ionized gas created when a material is "subjected to extreme heat, pressure or electric discharges" and undergoes ionization [2]. Electrons are stripped from the atom and a mixture of positively charged ions and free moving electrons results. The Aurora Borealis, neon signs, the ionosphere, and solar winds are examples of plasma.

Nibhanupudi et al. use a model called the plasma Particle In Cell simulation model to describe the electrostatic interactions of the charged particles in the spatial domain in 
relation to time. Specifically the researchers study the effects of injecting a weak low density electron beam into a high density plasma, introducing instability in the plasma waves. The authors transformed the original model to the General Concurrent Particle in Cell (GCPIC) model, originally intended for partitioning particles and related grid points to the processors of a MIMD distributed memory machine. Nibhanupudi et al. use the Bulk-Synchronous Processing (BSP) model to apply the plasma simulation algorithm to a network of workstations.

This plasma simulation could be easily implemented using the job pool paradigm. The interaction between the moving particles and the field surrounding it need to be calculated along its trajectory. The moving particles are the object of the calculation and therefore are primarily the cause of the load imbalance. Occasionally, the field information changed by the passage of particles in portions of the grid must be combined and redistributed to the processors. Also, as a particle passes out of a field grid piece that a processor has, it needs to be handed off to a processor with the connecting grid piece. This maps well to the job pool paradigm in that each pool can contain a map of the entire grid space that gets updated with each deposit from a worker upstream. Workers servicing this pool can then obtain a particle and updated field grid information for its calculations. Alternatively, the lock master could potentially keep track of the entire updated grid for the workers. Using the BSP model of the authors, measures must be taken to eliminate particle redistribution because of the 
communication effects, but with the job pool paradigm doing particle redistribution via the job pools is an advantage.

The load balancing for a plasma simulation job is taken care of inherently by the job pool, faster or unloaded machines return to the pool more often to get another particle for calculations. The calculations could be broken up into parts or the field grid could be divided to define a number of individual job pools. Particles and pertinent grid information make up the tasks in these pool.

A particle task is returned to the same job pool as long as the particle remains in the grid area supported by that job pool, when the particle moves out of that area, it is then

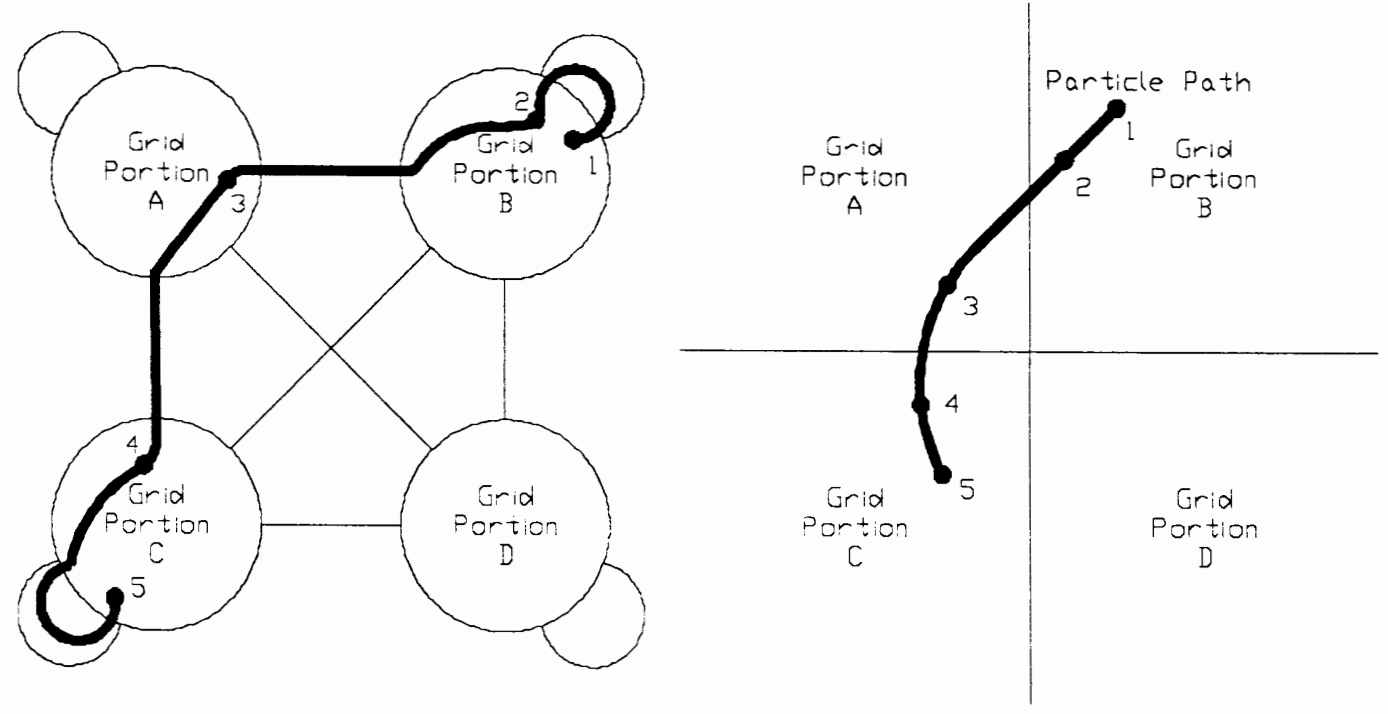

Figure 3-2. Possible Movements of Particles among Job Pools

deposited into the job pool corresponding to the next area. This was another area of difficulty for the BSP model, the authors used a buffer system to account for particle 
movement. Figure 3-2 gives an example of a grid divided among four job pools. This is an excellent example of a use for the conditional primitive in that the destination job pool depends on a particle's position after the calculations.

The migration ability of the workers in the job pool paradigm is not expressly used in this example, though migration could prove to be pivotal in simulation circumstances. Suppose a second phase of calculations, taking longer than the initial calculations, were done with each movement of the particle and this phase was defined with another job pool. If the initial calculations were quick, the particle tasks could pile up at this subsequent job pool. The workers servicing the movement pool could migrate, temporarily, to this calculation pool, until the it was caught up. this would reduce the bottleneck potential caused when one stage of a pipeline of job pools takes much longer than earlier stages.

\subsubsection{Genetic Database Alignment}

Another computationally intensive job is database searching. Comparing every entry in a database to a sample piece of information until a match is found can use an unlimited amount of computer resources. With luck a match could be found quickly, but with a large database this is unlikely. For example, comparing a human chromosome, which contains about 5000 sequences, against a database of 3,000,000 sequences is estimated to take a workstation up to 5000 days or more to do [1]. The advantage of distributed 
computing is clear, if multiple workstations can search different parts of a database simultaneously, total search time can be drastically reduced.

Using a genetic database to identify similar sequences is useful in determining the function and structure of an unknown DNA sequences. Sequence analysis has important applications in research such as the Human Genome projects. Mapping the human genome is a launching point for much genetic research involving diseases and defects. The work by Strumpen [1] in molecular biology sequence analysis involves a genetic database composed of 106,684 genetic sequences (126,212,259 bases). There were two search items chosen by Strumpen, one was 105 bases and the other was 773 bases. Strumpen uses over 800 workstations over the Internet to do the searches, reducing the search time from several days to a few minutes.

Most researchers probably do not have access to this many machines, and the job pool paradigm provides a simple framework to get the most out of the machines available. To apply database problems to the job pool paradigm a one-to-many primitive would break the database in the first job pool into pieces (i.e. tasks) and place them into the next job pool. Workers (one-to-one primitives) containing the search sequence would take tasks from this job pool, do the comparison and either place the non-matching result in a discard pool or place the partially matching portion into a different pool. There are many opportunities to separate the pieces of the database contained in the task according to how well the sequence is matched and to what portion of the sequence 
matched. In the simplest example, the non-matches are thrown out and the match results are sent to a third job pool where a many-to-one worker correlates and presents the results. The load balancing features of the job pool paradigm is especially useful since more available machines will take on more work than loaded machines, spreading the work out such that no one machine causes a serious bottleneck.

\subsection{Detailed Example: LU Decomposition with Back Substitution}

The application chosen for experimentation in this thesis is a sequential LU decomposition with back substitution. LU decomposition was chosen for several reasons. First, the $\mathrm{LU}$ decomposition and back substitution can be rearranged into several different forms of interest during experimentation. For example, the LU decomposition can be done in conjunction with back substitution in the same job pool or it can be split apart for a pipeline job pool scenario. Second, the emphasis is on the utility of the job pool paradigm and the LU decomposition is a straight forward mathematical implementation that does not require prior knowledge of genetics, plasma simulation, or any other specialized subject. While LU decomposition is a generic mathematical calculation, it still represents simulation in a specific area as the matrix and solution vector are composed of a set of equations describing a system or situation. Third, LU decomposition with back substitution, as used in the second set of experiments, is not "embarrassingly parallel," it is a serial application being applied to a distributed system. 
LU decomposition is a technique used to solve the matrix equation $\mathrm{Ax}=\mathrm{b}[42,43]$, where $\mathrm{A}$ is a nxn matrix and $\mathrm{x}$ and $\mathrm{b}$ are $\mathrm{n}$ length vectors. This technique breaks the $\mathrm{A}$ matrix into two easier to handle matrices, $\mathrm{L}$ and $\mathrm{U}$ as follows:

$$
\begin{aligned}
& \begin{array}{lllll}
\mathrm{a} 00 \mathrm{a} 01 \mathrm{a} 02 & \mathrm{u} 00 \mathrm{u} 01 \mathrm{u} 02 & 1 & 0 & 0
\end{array} \\
& \mathrm{~A}=\mathrm{a} 10 \mathrm{a} 11 \mathrm{a} 12=\mathrm{U}^{*} \mathrm{~L}=\begin{array}{llllll}
0 & \mathrm{u} 11 \mathrm{u} 12 & 110 & 1 & 0
\end{array} \\
& \begin{array}{lllllll}
\text { a20 a21 a22 } & 0 & 0 & \text { u22 } & 120 & 121 & 1
\end{array}
\end{aligned}
$$

The $\mathrm{L}$ and $\mathrm{U}$ matrices can then be used to find the $\mathrm{x}$ solution vector through two computations:

$$
\mathrm{Lc}=\mathrm{b} \text { then } \mathrm{Ux}=\mathrm{c}
$$

The $\mathrm{b}$ vector is given and the $\mathrm{L}$ and $\mathrm{U}$ matrices are from the original $\mathrm{A}$ matrix. The $\mathrm{c}$ vector is temporary and is used to do the final calculations to find the solution, $x$.

In practice, an LU decomposition is computed for the A matrix then forward and backward substitution are performed. The forward substitution solves for $\mathrm{c}$ and is easily done by making the $\mathrm{b}$ vector the $\mathrm{n}+1$ column of the $\mathrm{A}$ matrix and including it in the calculations for LU decomposition. The following outline explains the calculations involved in doing LU decomposition serially:

$$
\begin{aligned}
& \text { for } k=0 \text { to } n-2 \\
& \text { for } \mathrm{I}=\mathrm{k}+1 \text { to } \mathrm{n}-1 \\
& \mathrm{l}[\mathrm{i}][\mathrm{k}]=\mathrm{a}[\mathrm{i}][\mathrm{k}] / \mathrm{a}[\mathrm{k}][\mathrm{k}] \\
& \text { for } \mathrm{j}=\mathrm{k}+1 \text { to } \mathrm{n} \\
& a[i][j]=a[i][j]-1[i][k] * a[k][j]
\end{aligned}
$$


The $\mathrm{j}$ loop goes to $\mathrm{n}$ rather than $\mathrm{n}-1$ because the $\mathrm{b}$ vector is added to the matrix. As the loop structure is three deep the calculation is of $\mathrm{N}^{3}$ executions complexity. Thus both LU decomposition and the forward substitution are $\mathrm{N}^{3}$ executions hard.

Backward substitution, a $\mathrm{N}^{2}$ executions problem, is then used to find the $\mathrm{x}$ solution vector. The following algorithm outlines the method of back substitution:

$$
\begin{aligned}
& \text { for } k=n-1 \text { to } 1 \\
& \qquad \begin{aligned}
x[k]=c[k] \\
\text { for } I=k+1 \text { to } n-1 \\
\quad x[k]=x[k]-a[k][i] * x[i] \\
x[k]=x[k] / a[k][k]
\end{aligned}
\end{aligned}
$$

This algorithm assumes that when LU decomposition is done the results are written back into the A matrix as follows:

$$
L U=\quad \begin{aligned}
& \mathrm{u} 00 \mathrm{u} 01 \mathrm{u} 02 \\
& 110 \mathrm{u} 11 \mathrm{u} 12 \\
& 120 \quad 121 \mathrm{u} 22
\end{aligned}
$$

The $\mathrm{c}$ vector overwrites the $\mathrm{b}$ vector and the $\mathrm{x}$ solution vector in turn overwrites the $\mathrm{c}$ vector. This is ideal for programming since only one matrix structure and one vector structure are needed. If the original $\mathrm{A}$ or $\mathrm{b}$ need to be saved the algorithm can be adjusted to write the solution elsewhere. The experiments in this thesis write over the original values. 
As mentioned above, there are several ways the LU decomposition can be mapped to the job pool paradigm. The LU decomposition and the associated back substitution can be lumped together as generic work, in a function parallel situation. All of the tasks in the original job pool contain a matrix and $b$ vector that will be overwritten by the LU matrix and the solutions vector by a worker during calculation. A one-to-one worker takes a task, or tasks, does the LU decomposition with back substitution and places the resulting matrix and vector into the next job pool. To apply the MPMW model, a second set of workers serviced the second job pool, taking the once-calculated tasks and performing another LU decomposition with back substitution before placing the resulting task in a done pool. In this instance the LU decomposition is generic work, representing any set of tasks requiring no communication interaction.

Since both job pools perform the same tasks, this situation is convenient for looking at the migration abilities of the workers. The task size each pool handles is directly related to the size of the matrix, thus one pool can be made computationally larger than the other by giving it much larger matrices to work on. This computationally large pool would become a bottleneck and workers would migrate to relieve it. Thus the effects of migration can be studied.

Another formulation puts the LU decomposition function in the first job pool and the back substitution in the second job pool. This can be implemented in two ways, either a one-to-many worker can do the LU decomposition and place the resulting LU matrix 
and a solution vector in each task deposited in the second pool, where a separate one-toone worker does the back substitutions or a one-to-one worker does the LU decomposition on a matrix, leaving the solution vector alone, for back substitution by the second one-to-one worker. The scenario in the first case is a situation where the LU matrix from the first pool is used with many different solution vectors. In the second approach, computationally the same, a single solution vector is associated with a single matrix in a task. This is the approach used in this thesis. 


\section{Chapter 4}

\section{Results and Discussion}

For system measurements and model verification, the chosen application was LU decomposition. In the first part of the experiment, each task is one decomposition, including back substitution, so an entire job was composed of several hundred separate LU decompositions. The two job pools are identical, thus a task will undergo two LU decompositions before reaching the done pool. At this point the two job pools are essentially identical, except that the first job pool, pool A, starts containing the total number of tasks. Thus pool A will have no arrivals while pool B will continually receive arrivals from workers servicing job pool A. In making the pools identical the calculations are simplified to provide a first look at the job pool paradigm and how it compares to the model.

In the second portion of the experimentation, workers will perform the LU decomposition on tasks from pool A with the results going to pool B. Workers servicing job pool B will then perform the back substitution on those tasks. This scenario represents a situation where the job pools are unequal, where one pool becomes a bottleneck. This is where the migrating workers of the paradigm become a real advantage. More workers can service the bottleneck pool, relieving the problem.

In both experimental setups, system data about task computation times (te), resource busy times (B) and spinlocks (Vs) for each pool must be gathered for use in the model. 
In practice, this would be done in a "test" run before the full run, so the values could be used in the MPMW model to predict run time performance. In this experimental setup, however, the measurements were continually taken as the program executed instead. This was done for several reasons. First, for the number of experiments performed, it was much more effiecient (on the system) to do the run and take measurements at the same time. Secondly, recording the system measurements throughout allowed monitering of the system to ensure that no unexpected behavior occurred. Third, using the measurements from a run in the model to test the predictive value of the model eliminated some system variation that would occur between a test run and a full run.

Before discussing the experimental results, the computer network under test must be examined. Seventeen Sparc 5 workstations running Solaris 2.4 were used in the experiments. Since the MPMW model takes system usage into account, the machines used were not restricted from general use during experimentation. System usage is calculated from processor idle time, thus the effects of ethernet traffic in the network is not considered. To minimize the effects of the ethernet traffic, programs runs were done during non-peak hours when possible. 


\subsection{System Usage}

The processor idle time of five "typical" processors were studied for 3 months to determine average system usage for our system. The UNIX command "vmstat" returns several pieces of information including processor busy time, system time, and idle time. Using the UNIX "cron" utility, vmstat was called on each of the five processors every hour for three months. The data was collated to give average percent idle times per hour for each day of the week.

From the processor idle percentages, the system usage is calculated. For example, if idle time is $90 \%$ then the system usage term is 1.1 . This is the number to multiply the cpu time by to account for the $10 \%$ of time the processor is not available. Thus the system usage will be one for a dedicated server and will increase as necessary to correct for the increase in computation caused by sharing the processor with other user's and applications. Figure 4-1 gives an example of the average idle time of a machine called

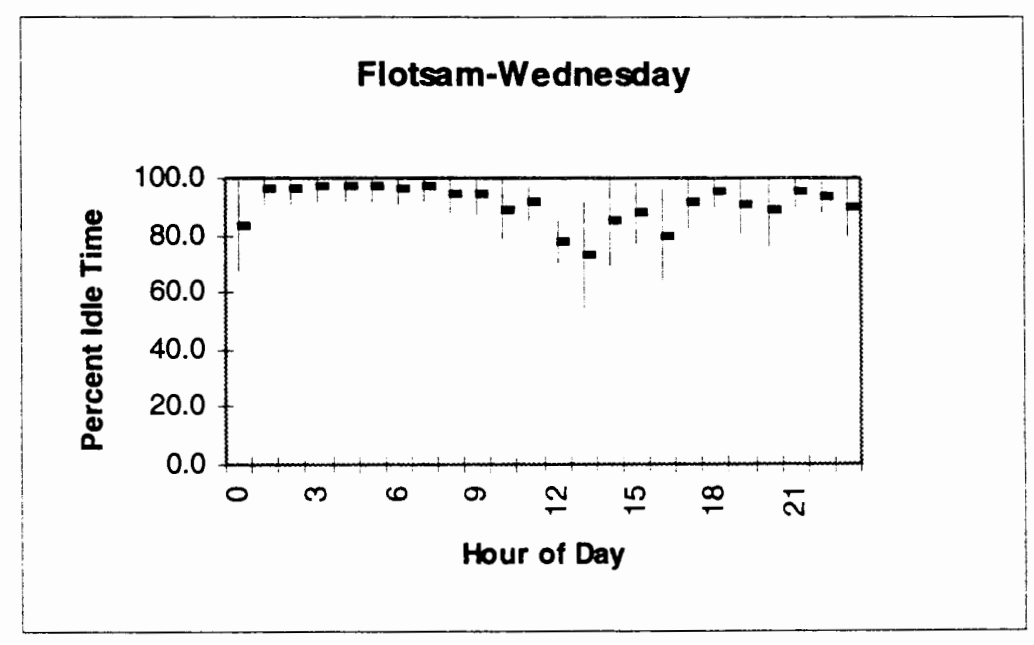

Figure 4-1. Flotsam on Wednesdays. 
flotsam for every hour on a Wednesday.

Figure 4-1 shows that the period of time between 2:00pm (14:00) and 7:00pm (19:00) on Wednesday is the busiest and running the test program on this machine should be avoided during this time . Even though the processor idle percent is still at around 85\% during the busy periods, running the test program will have an effect on other users of the machine. The other workstations have similar busy periods, thus an experiment may be started at midnight, when the system usage term is lower.

Rather than look at each machine individually, the percent processor idle time of the five processors is averaged over three months to form a system percent idle time. From the system percent idle time, the system usage is derived for the models. Since percent idle time gives the fraction of time that the system is available for our use, the system usage is

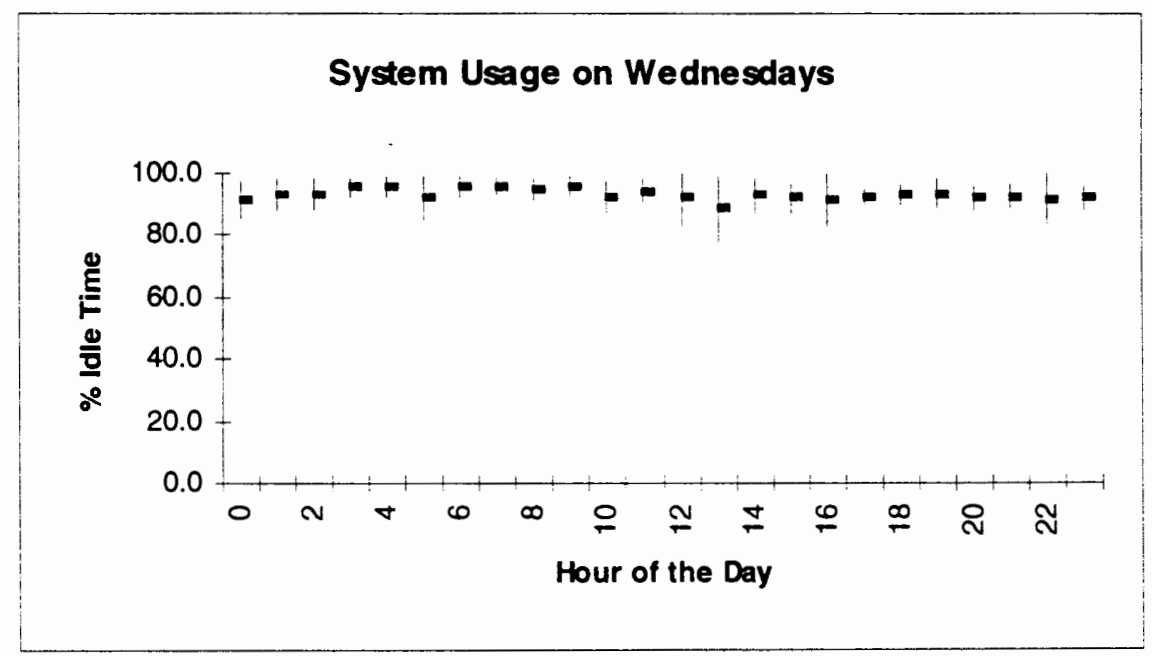

Figure 4-2. 
a multiplier accounting for unavailable system time in our model. If the system percent idle time is $75 \%$, for example, then the system usage would be 1.25 . The system usage for each hour on Wednesday is given in Figure 4-2. Similar charts have been derived for each day of the week. The time and day of each execution of the test program is noted so the appropriate system usage may be used with the MPMW model.

When running experiments, data collection using vmstat is continued to insure that an unusually large system load does not produce error in the data collection. Therefore if the percent idle time at midnight on Wednesday is reduced to $60 \%$ by other users, errors can be avoided from underestimating (or overestimating) the system usage. In practice a user might want to check the system usage when the program starts and use that as an estimate. Obviously, if the system load increases, dropping idle percentage times, a distributed program takes longer to run.

Using MPMW paradigm, the effects of a single machine having decreased percent processor idle time is minimized. If a machine becomes loaded down, the worker on that machine may simply take fewer bundles of tasks during program execution. Machines with more idle time would handle more bundles, evening out the effects and balancing the load, though overall execution time would still increase. 


\subsection{Phase 1 - Equivalent Job Pools}

\subsubsection{System Measurements}

As mentioned above, during the first phase of experiments, the two job pools are equivalent to simplify calculations and comparisons. A full LU decomposition with back substitution is performed by a worker on each task from each pool. Thus a single task file, containing a matrix of size $n$, will undergo two LU decompositions. Each execution of the distributed program involves 100 tasks. Rather than changing the number of tasks during the experimentation, the size of the matrix in the task is changed to alter the amount of work in the system. The two matrix sizes used are 50x50 and 100x 100. The $100 \times 100$ matrix was the largest used to keep execution time manageable, especially considering the number of times the program was run. The 50x50 matrix was used for two reasons; to show the effects of changing the problem size, and to emphasize the serial costs of distributed computing.

For each problem size the test program was executed using first a single processor, then using $3,5,7,9,11,13,15$, and 17 processors. Also to investigate granularity, a major concern of this thesis, several bundle sizes for each problem size and set of processors was used. By definition granularity is the bundle size, so if workers are retrieving ten tasks at a time from a job pool, then the granularity of that job pool is 10. For each problem size, at very processor number step, the program was executed at granularities $1,10,20,30,40,50$, and 100 . Using the granularities 1 and 100 establishes boundaries 
on performance, with a granularity of 100 being a serial scenario, since there are only 100 tasks and thus only one worker in use. Granularities above 50 should give no further information, since with 100 tasks only 2 workers need service the job pools in these cases. Finally, each individual test run is done five times to insure that average behavior is obtained. A table listing experiments is given in Appendix B.

As discussed in Section 2.3, runtime measurements were made for each test run compiling execution times, task computation times, pool locking times, pool unlocking times, and number of lock attempts for each worker. The execution times are for comparison to the MPMW model and for discussions of scalability and granularity, the other measurements are used to determine system values needed by the model. For example, the resource busy time, $B$, is the sum of the pool lock time and the pool unlock time. In terms of our model, the measured task computation time is actually the task process time, te, multiplied by the number of completions, $\mathrm{C}$. The number of lock attempts is the spinlock count, $\mathrm{V}_{\mathrm{S}}$ in our model.

\subsubsection{Execution Times}

Since execution time is of foremost concern to most distributed programmers, it is examined first. The test program does a LU decomposition, with back substitution, for each task in each pool, thus 200 total LU decompositions are performed at each program execution. The experimental execution time is examined as the number of processors used, problem size, and granularity change. Since only two problem sizes 
are examined, the results from using each size are considered separately before a comparison between the two is made.

The first set of experiments deals with 100 tasks each containing a 50x50 matrix to under go LU decomposition in each of the two job pools. Figure 4-3 shows the execution time verses the number of processors and the granularity. $T_{\text {Actual }} / \mathrm{N}$ is a simplistic model used for comparison that does not take overhead into account.

Since the model developed in this thesis does not account for the starvation of workers when high granularities are used, program executions with these higher granularities were not done using excess processors. Thus since only 4 workers are needed at once when the granularity is 30 , experimenting with 11,13 , etc. processors provides no more information. The test results show that program executions using higher granularities

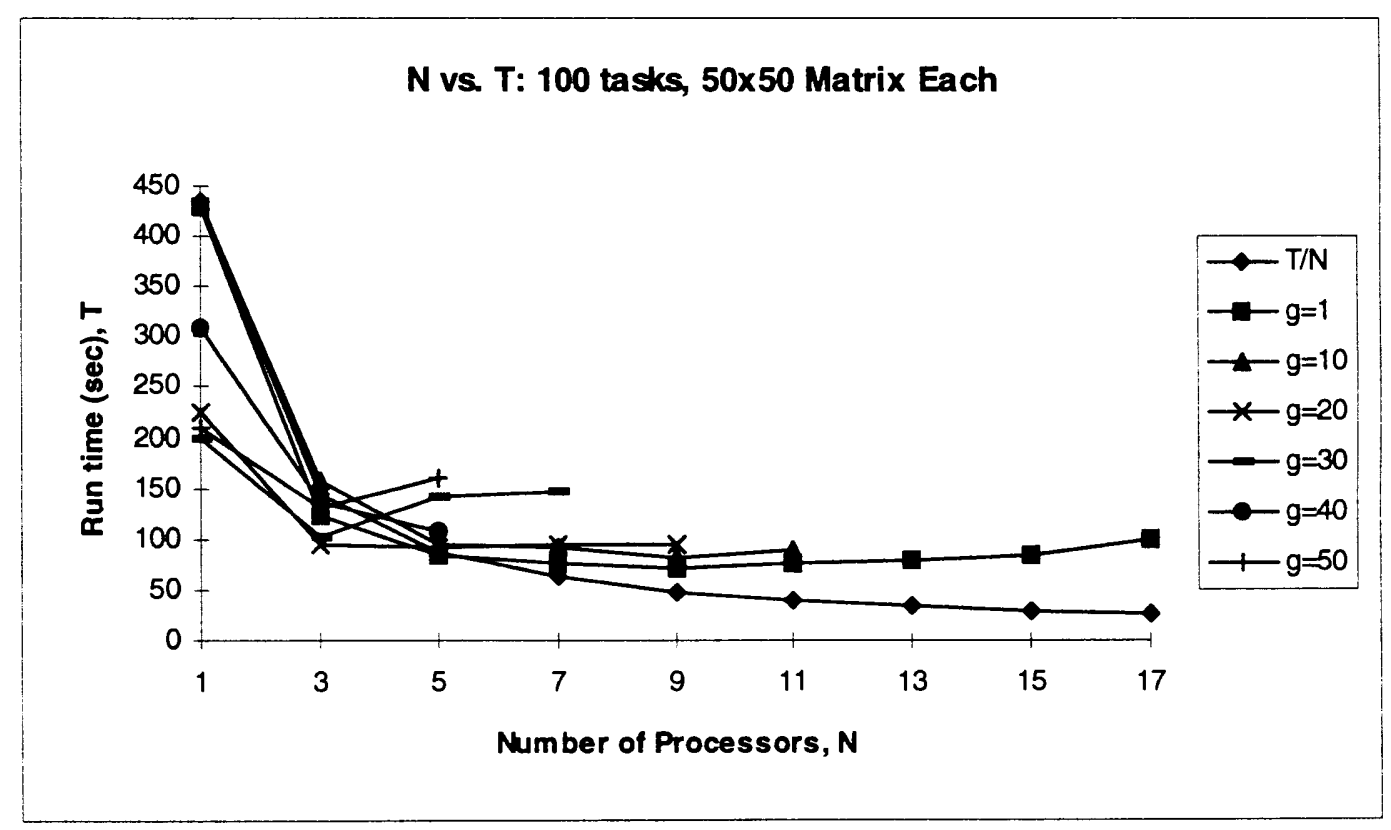

Figure 4-3. 50x50 Matrices 
tend to have higher execution times than the runs with lower granularities when more than 3 processors were used. Figure $4-4$ provides a three dimensional view of the relationship between the number of processors, the granularity and the execution time of the program.

An important feature in both Figures 4-3 and 4-4 is the upsweep of the execution time when using a large number of processors at low granularities $(\mathrm{G}=1)$. This increase in execution time is expected as increasing the number of processors drives up overhead costs, ruining performance. The decrease in performance is not dramatic at 17 processors, but the effects would increase if more processors were added to the system. Although increasing granularities does not increase performance, the increase in execution time at the higher numbers of processors is not seen. This is not surprising

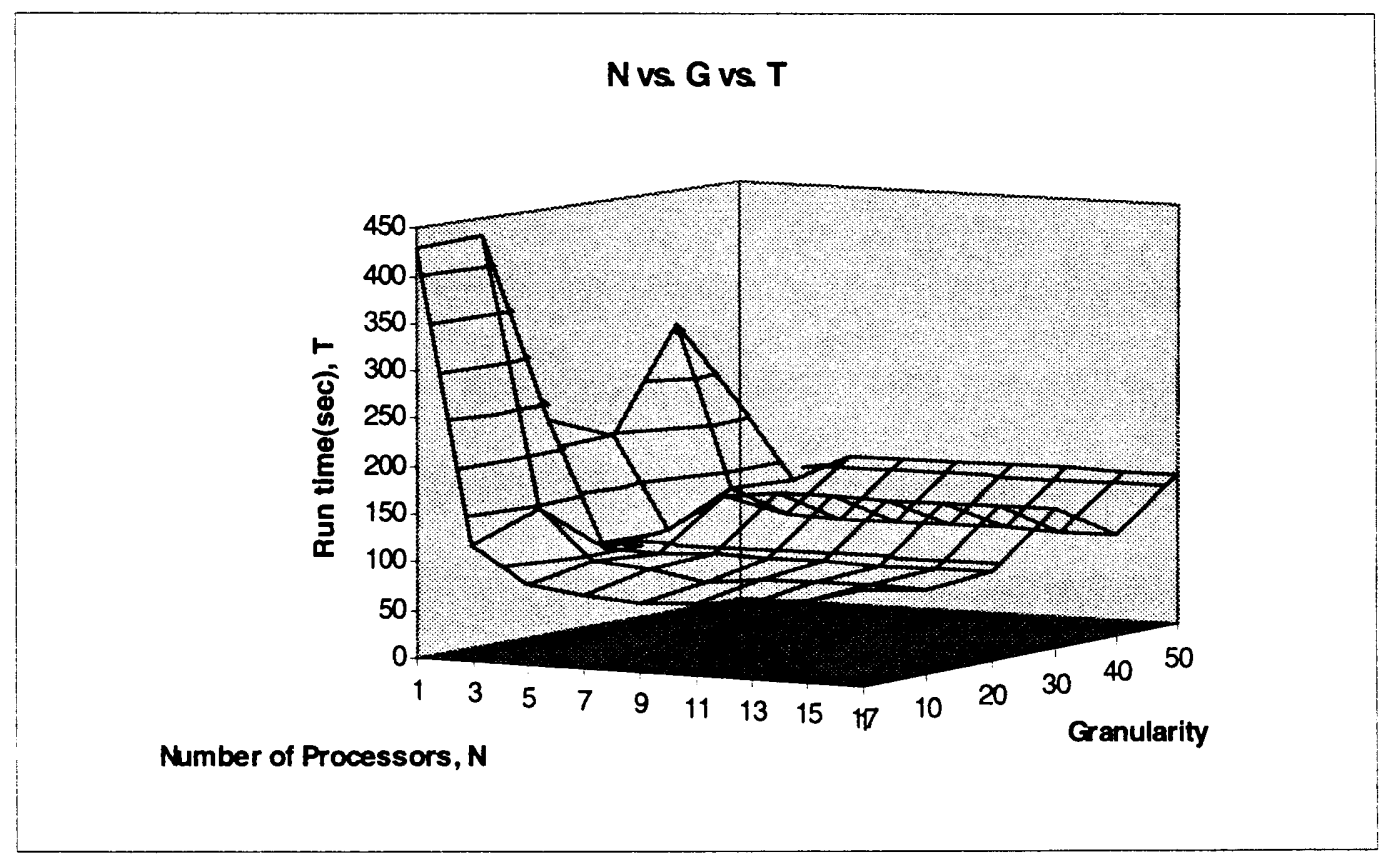

Figure 4-4. 50x50 Matrices 
since the communications overhead is reduced as granularities increase, because workers have to visit the job pools less at higher granularities. However, using higher granularities decreases the benefits of the load balancing provided by the job pool paradigm by reducing the number of pieces a job is broken into.

When the larger task size, $100 \times 100$ matrix size, is used the execution time results in Figure 4-5 are seen. In this set of test runs, all the granularities were taken further out to insure that no unexplained changes occur after the maximum number of usable processors is reached. An additional set of test runs were ran with a granularity of one hundred to provide a serial example for comparison.

The results in figure 4-5 clearly indicates that for tasks of this size, increasing the

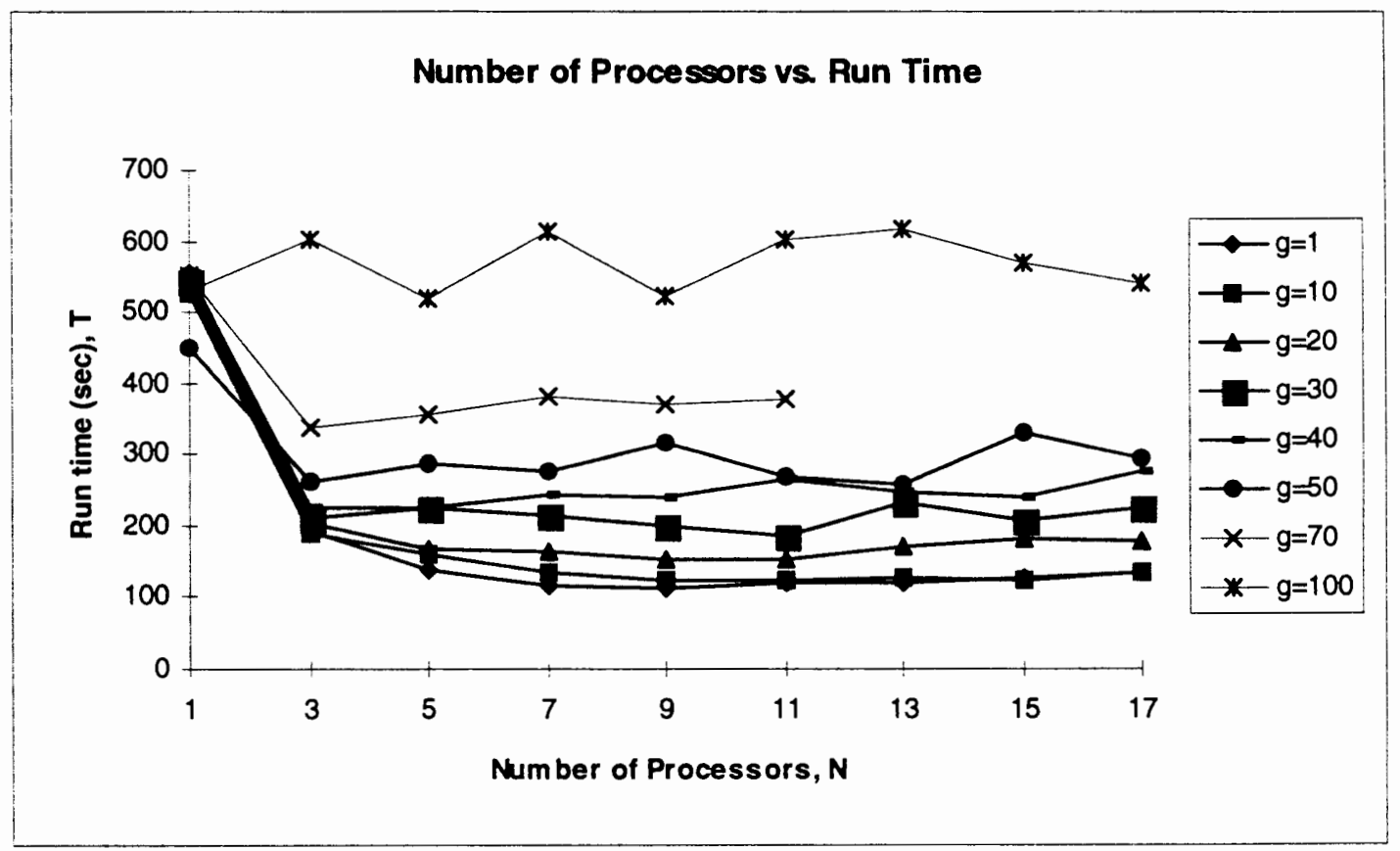

Figure 4-5. 100x 100 Matrices 
granularity past one increases the execution time, though a granularity of ten is close. From figure 4-3, using a granularity of one, ten or twenty tasks produce similar results,though a granularity of one has about the lowest execution time. It is likely that since the task size is smaller in the results from Figure 4-3 that a higher granularity could be used with no performance degradation. Unfortunately, using these higher granularities gives no benefit to either. The data indicates that whether or not changing the granularity increases performance depends on the size of an individual task. Using a granularity of 20 with a task size $50 \times 50$ produces a smaller performance hit than the 100x100 tasks. Potentially, systems with very small tasks could benefit from increasing the granularity.

\subsubsection{Task Computation Time and te}

Before examining how the experimental data compares to the MPMW model, it is

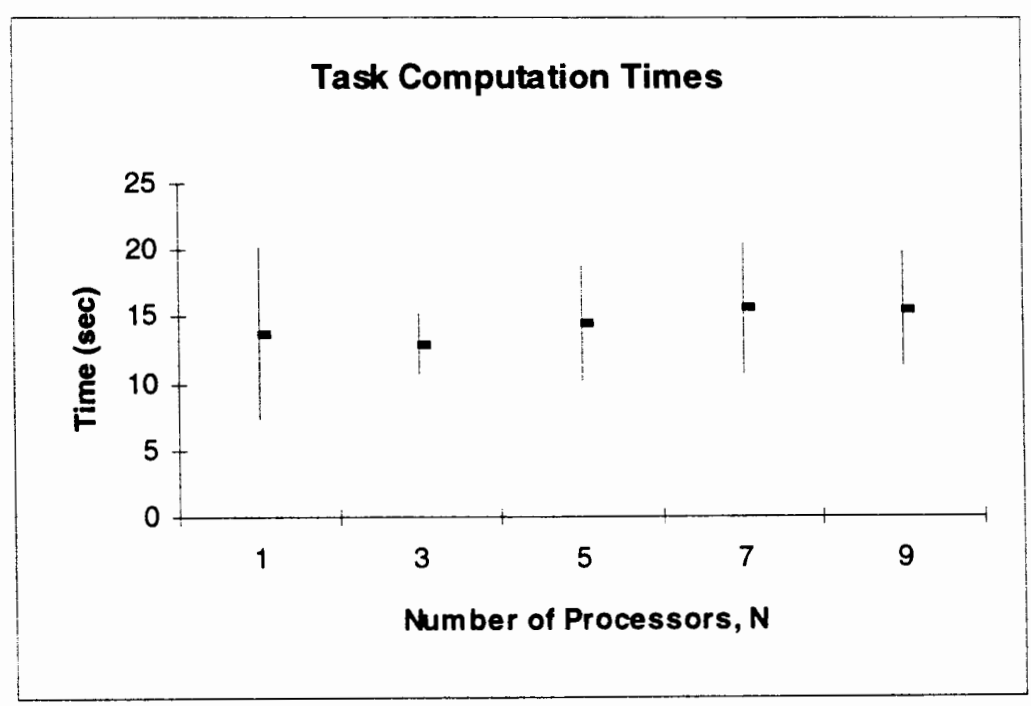

Figure 4-6a. 50x50 Matrices, $G=20$ 


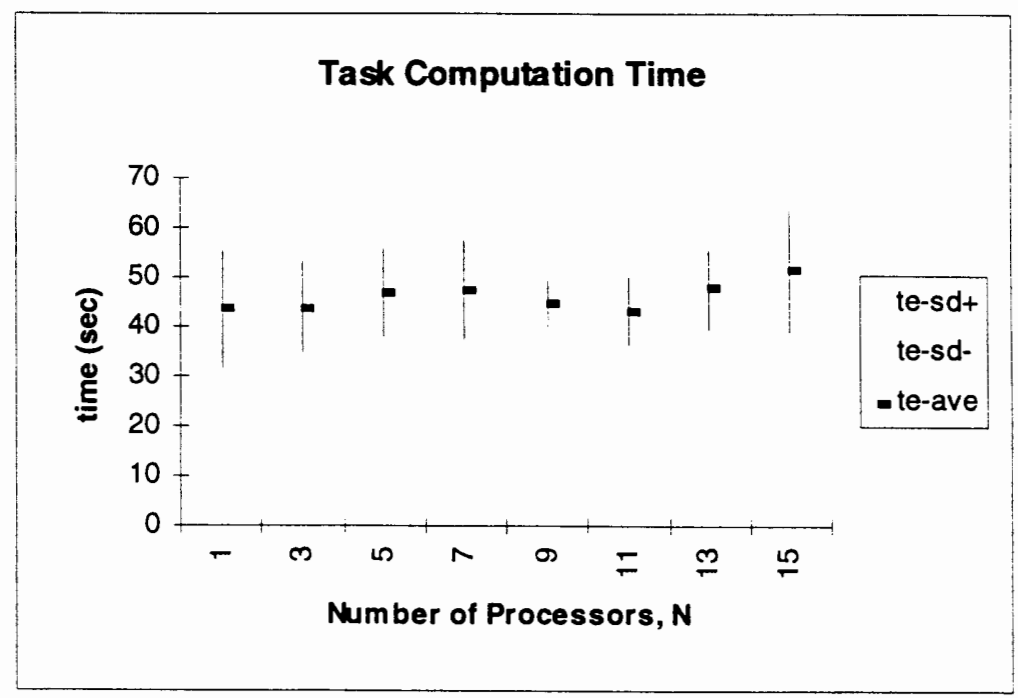

Figure 4-6b. $100 \times 100$ Matrices, $G=20$

instructive to look at the experimental values of task computation time, B, Vs, and execution time as granularity, problem size and the number of processors change.

Looking at task computation time as the number of processors change, for example, shows, as expected, essentially no variation. The time it takes for a worker (processor) to complete a task should not change as the number of processors increases.

Figure 4-6a and figure 4-6b assure us that this is indeed the case. Figure 4-6a illustrates the task computation time for 100 tasks, each containing a 50x50 matrix, at a granularity of 20 . Figure $4-6 \mathrm{~b}$ gives the results for 100 tasks containing $100 \times 100$ matrices, with a granularity of 20 . The biggest source of error in the task computation time is the change in system load, which is accounted for in the MPMW model by the system usage. 


\subsubsection{Resource Busy Time}

Unlike the task computation time, the resource busy time, B, varies as the number of processors used by the program increases. Figures $4-7 \mathrm{a}$ and $4-7 \mathrm{~b}$ show the relationship between the resource busy time and the number of processors when the granularity is

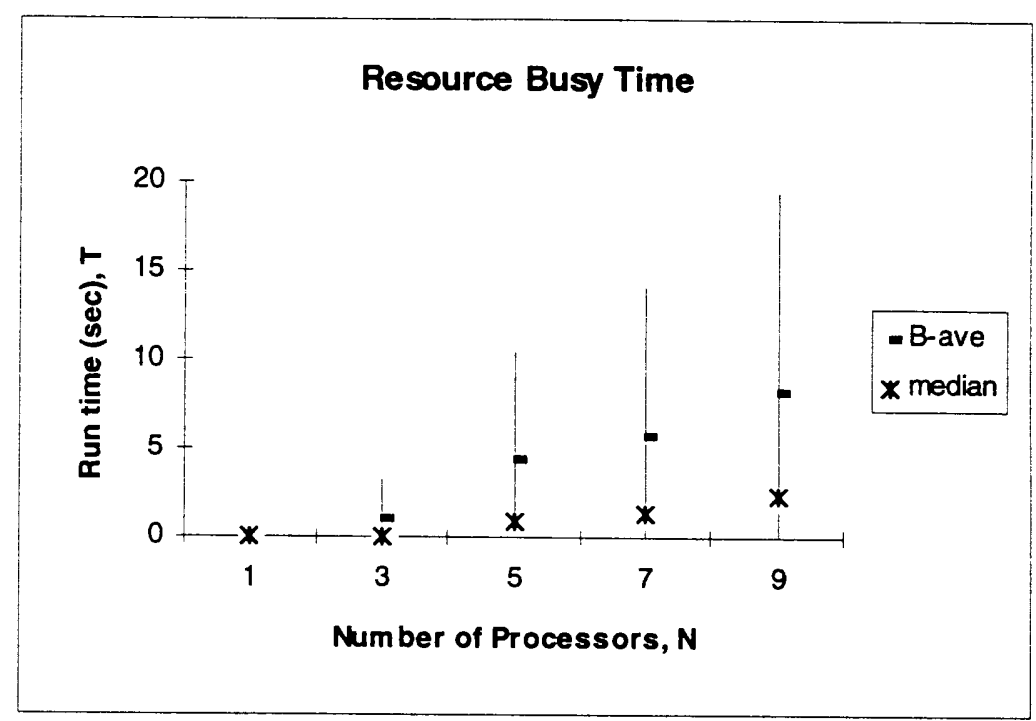

Figure 4-7a. 50x50 Matrices, $G=20$

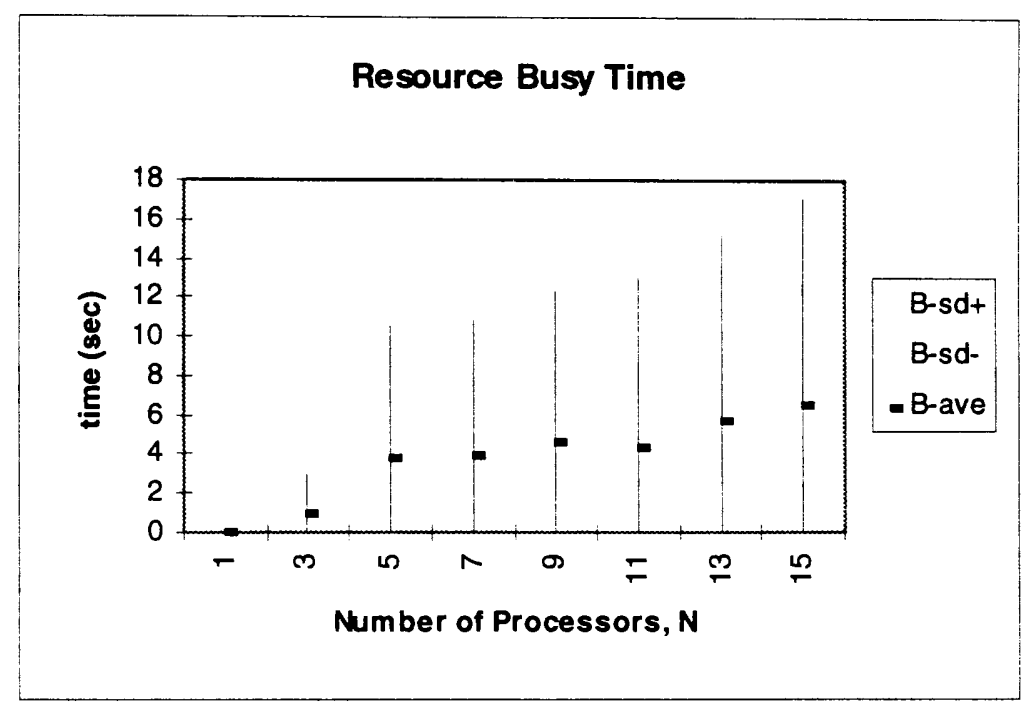

Figure 4-7b. 100x100 Matrix, G=20 
twenty. Data from program execution with other granularities produce similar graphs and a three dimensional representation of the resource busy time over all granularities and numbers of processors is shown in Figure 4-8.

The behavior in Figures $4-7 \mathrm{a}$ and $4-7 \mathrm{~b}$ is expected because as the number of workers increases, the competition for job pool locks also increases. When the competition for the locks increases a worker spends more time attempting to attain a lock, driving up the resource busy time. The time to unlock a pool stays fairly constant since no competition is involved and does not affect the increase seen in B. The value of B should be closely tied in to the number of times a lock is attempted, though section 4.2.1.4 shows this is not necessarily the case. If a worker only tries for the lock once, and gets it, then the value of B is small. If the worker attempts the lock several hundred times before successfully gaining it, then the value of $B$ will be quite large. This accounts for the variation seen in Figures 4-7a and 4-7b.

Figure 4-8 clearly show the cost of increasing the granularity on the system performance. it should be noted that Figures 4-8 reflect results from tasks of 50x50 matrices, thus there is a portion of the graph at $\mathrm{N}>11$ and $\mathrm{G}>10$ without data. The reason for this null portion of the graph was mentioned earlier; when the granularity is 40 , for example, anything above 5 processors is redundant. The upswing seen in Figure 4-8 with high numbers of processors and a granularity of one is the beginning of the performance 
deterioration resulting in the increase of serial time and execution time as seen in Figure 4-3. If more processors were added to the system the increase in resource busy time would continue, making the extra processors not only useless, but also detrimental. The resource busy time experiences this increase due to the increased competition from the larger number of processors. Increased competition also explains the dramatically

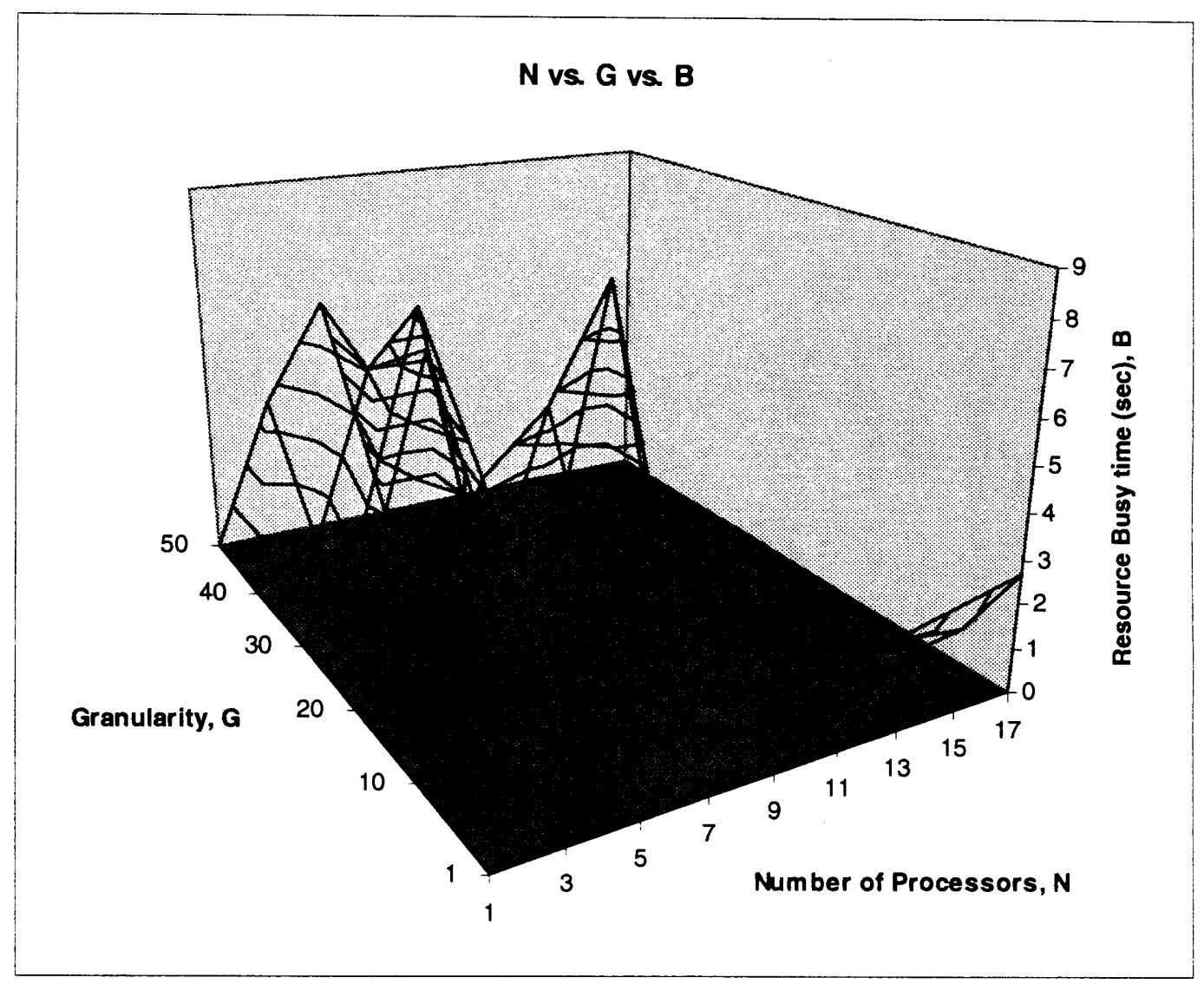

Figure 4-8. 50x50 Matrices

increased resource busy time seen at higher granularities with fewer processors. When the granularity is one, the are 100 bundles for the 15 or 17 processors to compete for, when the granularity rises to 30 , there are only four bundles in a pool for processors to 
service. At this point, excess processors take up resource time looking for data and blocking each other.

\subsubsection{Spinlocks}

As seen in figure 4-7a, the value of $B$ increases with the number of processors. The number of processors used increases, the competition for the lock increases and more failed attempts for the lock can be expected, driving up the resource busy time as shown in the figures. This is not entirely the case, however. Examining the number of lock attempts (or spinlocks) shows an increase with the number of processors, to a point, then a decrease. If the above comments are correct then the number of spinlocks should continually increase with processor number, but they do not. Figures 4-9a and 4-9b show the average number of the lock attempts before success as the number of processors increases for a granularity of 20. Figure 4-10 shows a three dimensional view of the number of spinlocks verses granularity as the number of processors increase. As with the resource busy time, no data was collected above 9 processors at higher granularities. This produces a flat area in the map that should be ignored.

As shown in Figures 4-9a and 4-9b, the number of spinlocks increases with processors until about 5 processors, then they begin to decrease, even though the resource busy time does not. Since the granularity is 20 and there are 100 tasks, it is not surprising that the turning point when 5 processors are used. In this example there would be five bundles each in pools $\mathrm{A}$ and $\mathrm{B}$, thus five processors are needed to do this work and 
deposit the tasks in pool B. At this point the excess processors will see that pool A is empty and continually check pool B for work. Since only five workers can be successful, the subsequent checks of pool A are quicker, providing less time for blocking which drives up the number of spinlocks. Five tasks will eventually flow

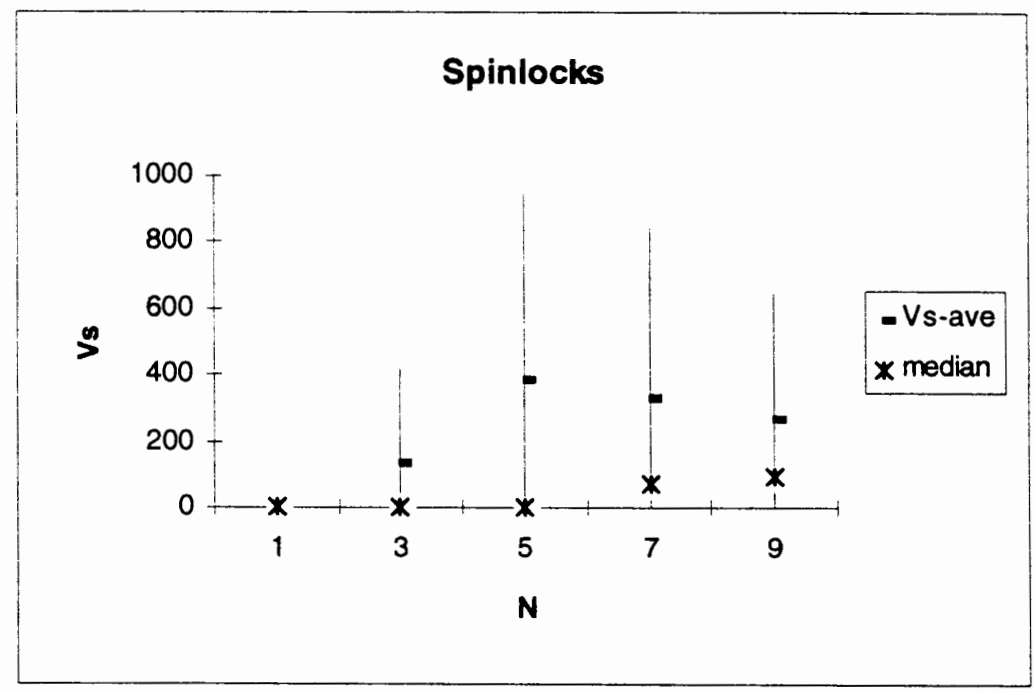

Figure 4-9a. 50x50 Matrices, $\mathrm{G}=20$

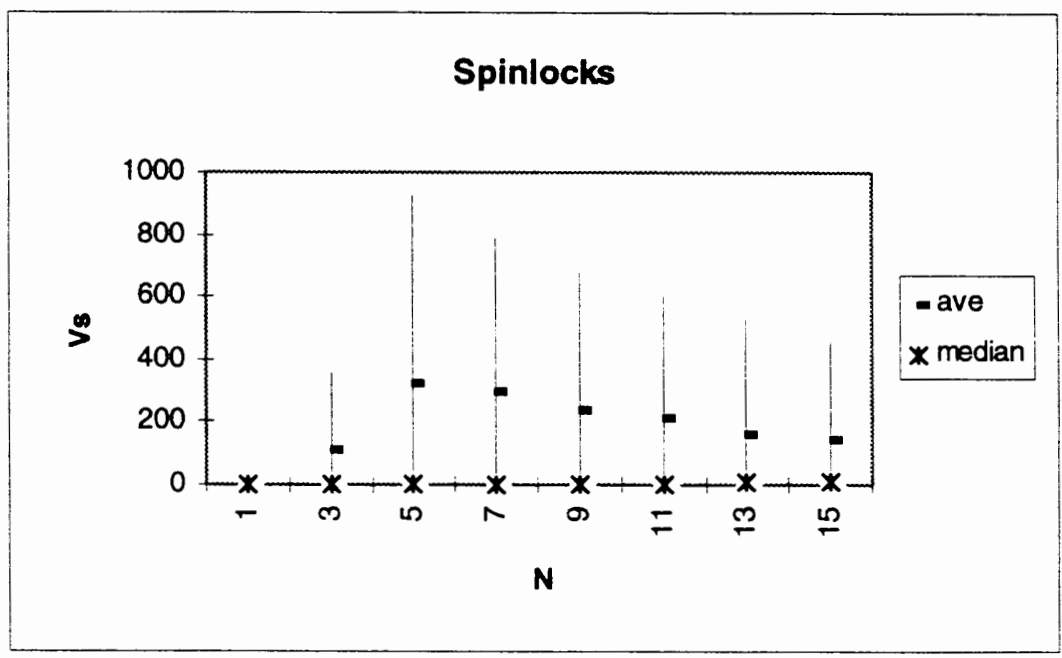

Figure 4-9b. 100x100 Matrices, $G=20$ 
through pool B, again requiring five processors to service them. If all the processors are similar, with similar loading, then all five will finish at approximately the same time, then the next (or same) five processors will attend to the bundles in pool B. When all the bundles are withdrawn from pool B, any worker attempting to get work from pool B sees that no work remains and exits the system. Again since only five processors can be successful, additional locks on pool B take much less time, reducing blocking and thus the number of spinlocks. Also, having more processors and much shorter service times allow the system to cycle through processors faster, potentially decreasing the

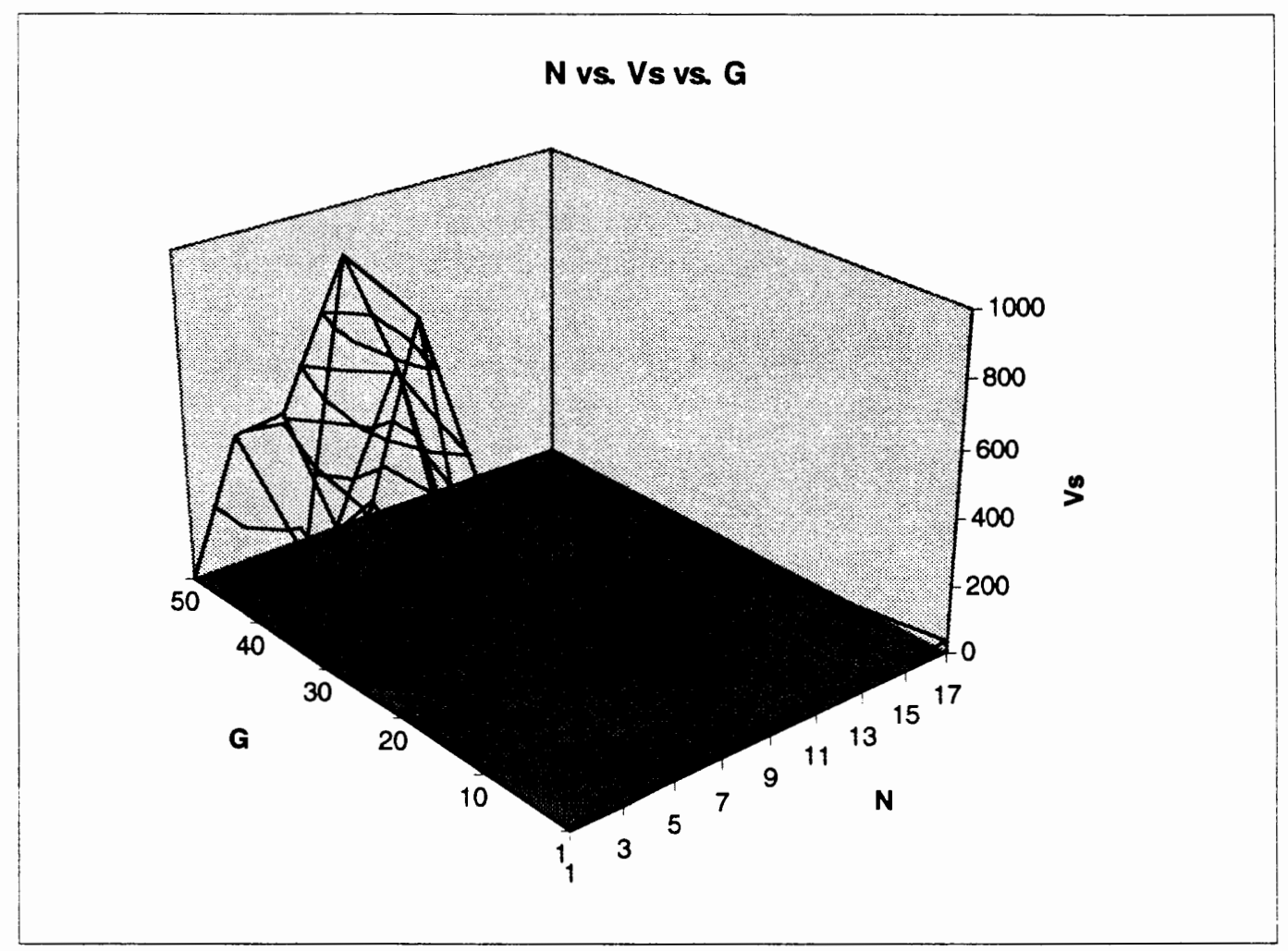

Figure 4-10. 50x50 Matrices 
spinlocks, a processor makes more visits, but takes fewer spinlocks to get there. Thus, a system with five processors provides the maximum possible competition for that granularity.

The large standard deviations seen in Figures 4-9a and 4-9b stem from the fact that a worker may obtain a lock on the first try or it may take several hundred attempts before success. Figure 4-11a assures that there is no place or function in the test program causing high values for spinlocks, Vs. The low values and high values of Vs are spred throughout execution without any discernible pattern. Similar results can be obtained from other granularities (assuming identical pools) and number of processors.

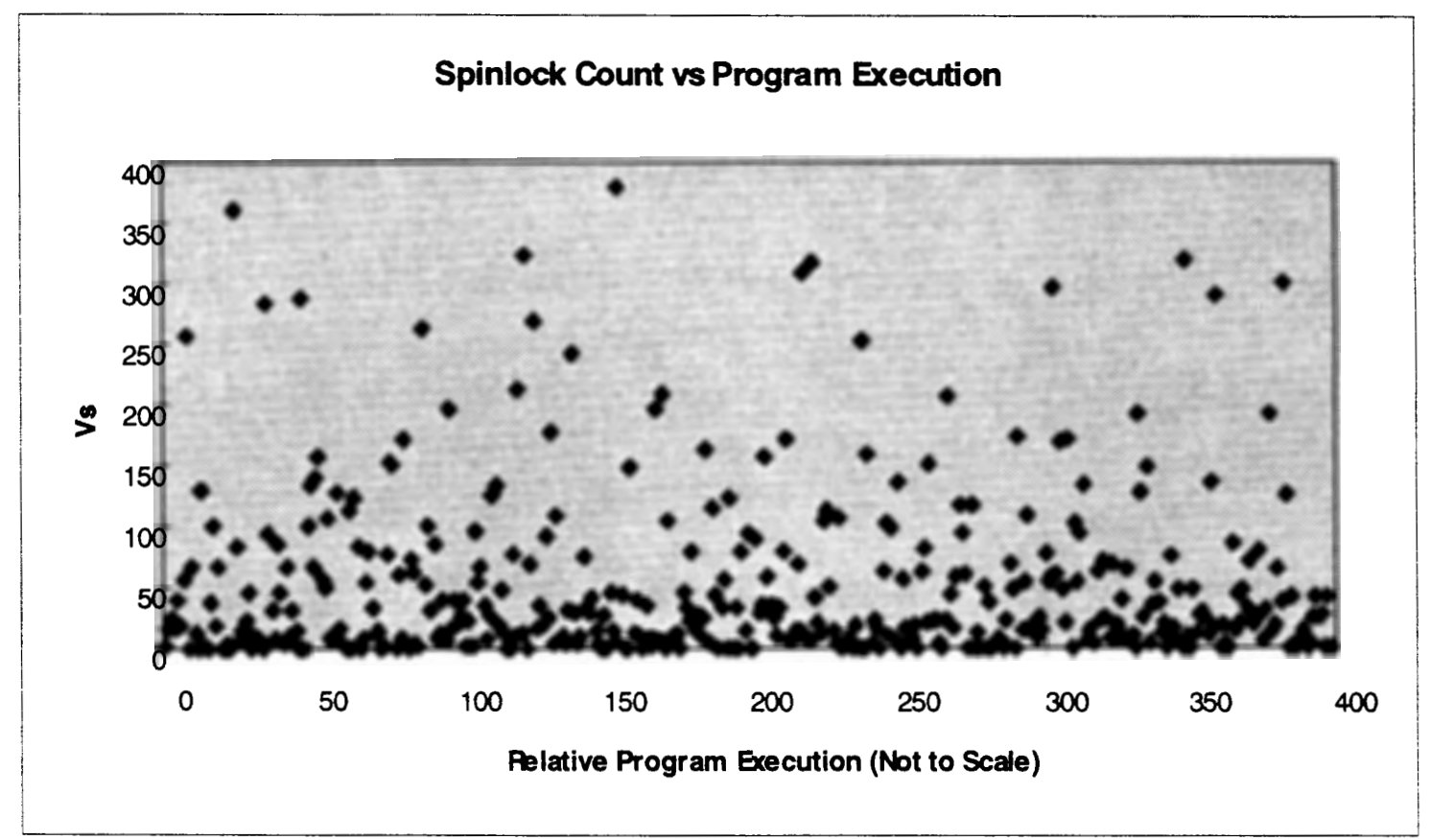

Figure 4-11a. 50 x50 Matrices, $\mathrm{N}=11, \mathrm{G}=1$ 


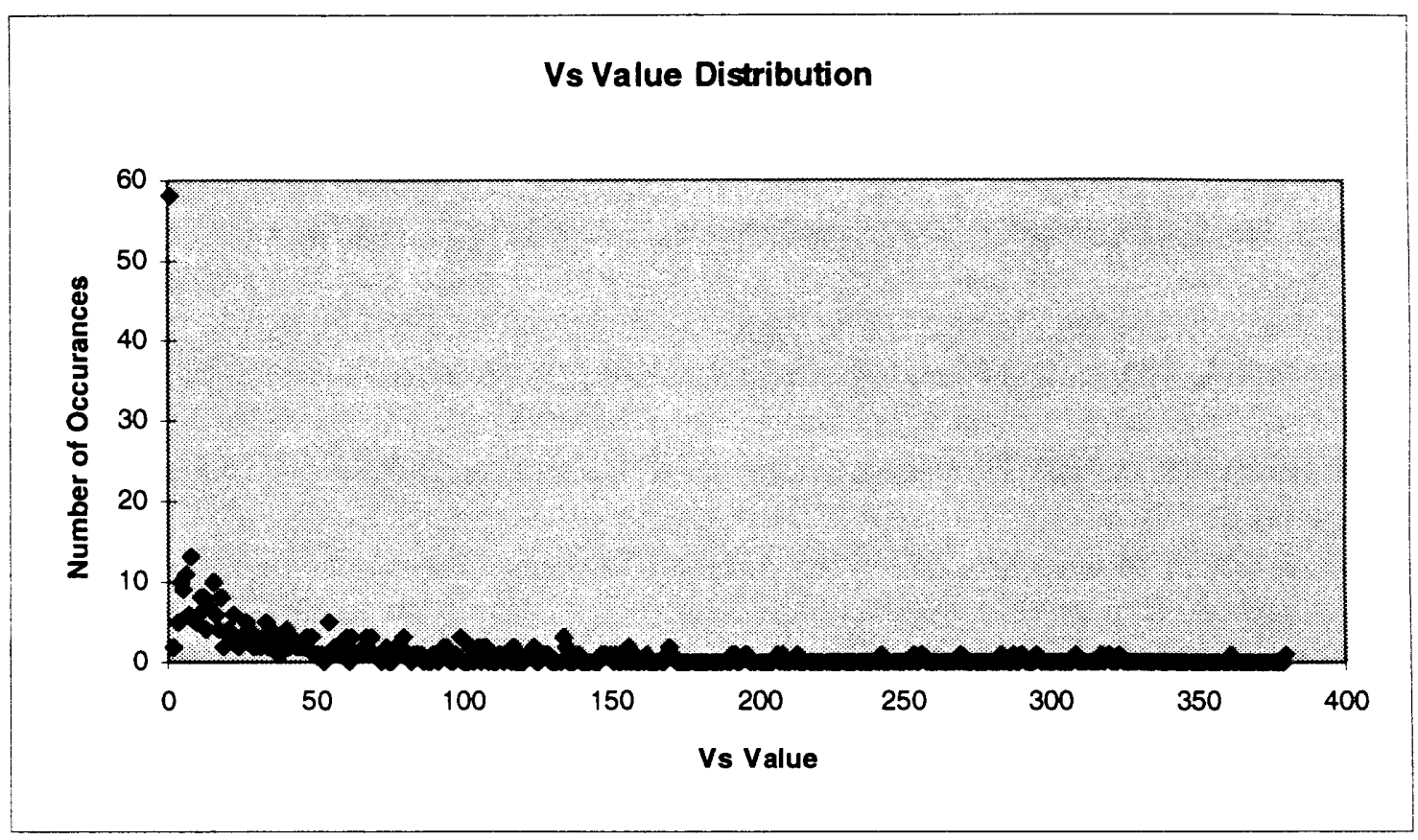

Figure 4-11b. 50x50 Matrices, $\mathrm{N}=11, \mathrm{G}=1$

Figure 4-11b expands the deviation from Figure 4-9a at eleven processors to show the distribution of spinlock values. As shown in this graph, the majority of the values are less than 100, in this example, but many high values of the Vs occur once or twice, inflating the average and the deviation. There are several possible reasons for this strange distribution. At any given point multiple workers can attempt to get a lock on a pool, the first one to request the lock from the lock master gets the lock. Thus the choice

of available worker to get the pool is random. This randomness means that an "unlucky" worker can fail many times before gaining a lock. Also, since there is a single lock master, the workers must actually compete for the lock master's attention. Thus, while the MPMW paradigm relieves conventional distributed system bottlenecks, 
a new bottleneck can be created at the lock master. A parasitic queue forms at the lock master as workers wait to place their requests.

\subsubsection{Scalability}

Figures 4-12a-d show how the test system scales with problem size and the number of processors as the granularity changes. In Figure 4-12a the granularity is one and the results for each problem size shows expected behavior, as discussed earlier. The interesting observation to note, is that as the granularity increases, the gap between the two curves increases. This gap shows the cost of increasing granularity is more detrimental with the larger task size. It was noted in the discussion of Figure 4-3 that increasing granularities with a small task size gives no advantage, but does not provide a serious performance hit either. Figure 4-5 showed a very decisive performance degradation as granularity increases. Overall the system scales better with smaller task sizes and smaller granularity. Bundling tasks according to granularity effectively

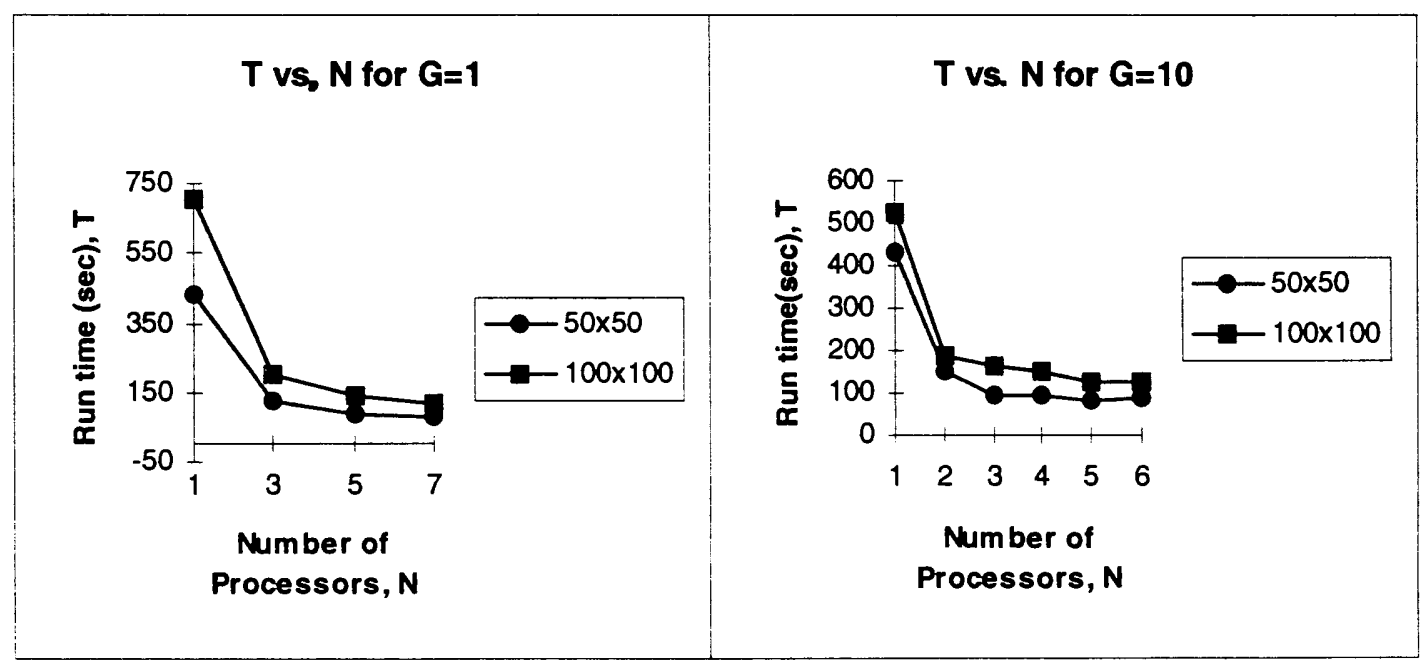

Figure 4-12a.

Figure 4-12b. 


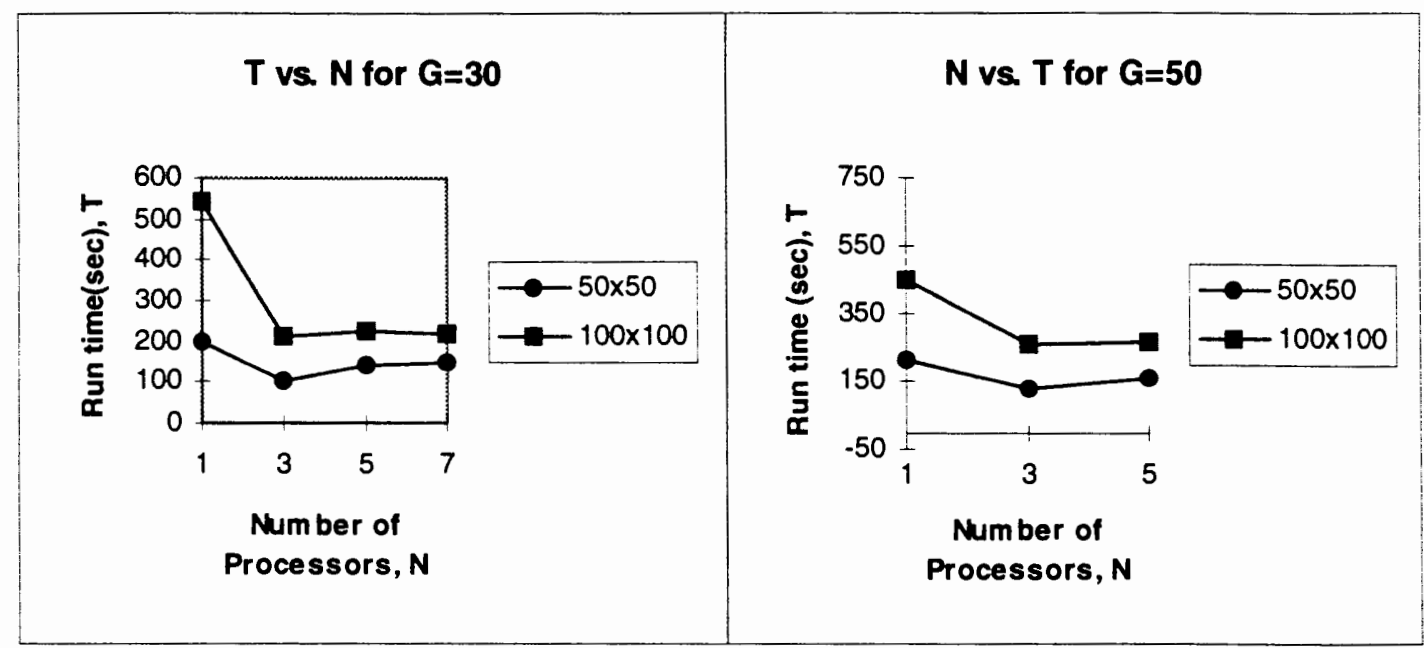

Figure 4-12c.

Figure 4-12d.

produces bigger tasks. Thus when considering what task size and granularity to use the overall size of the bundles must be taken into account. Bundling small tasks (smaller than a 50x50 matrix for LU decomposition) together may provide a performance gain, but small granularities must be used when dealing with larger tasks. The general trend shows that with a small enough task, increasing the granularity will give a performance gain. Finding a way to decide when to bundle tasks and how many to bundle is left for future considerations in Section 5.

\subsubsection{Experimental Data verses Models}

In order to use the MPMW model to predict execution times the system measurements discussed in section 4.2.1 are needed. In practice, a test run of a distributed program would be needed to estimate the task computation time, the resource busy time and the number of spinlocks for use in the model. To examine the model carefully, the system measurements were taken directly from program runs where the execution time was 
recorded. This allows a direct comparison of the MPMW model's prediction of execution time to the actual execution time as shown in figure 4-13a and 4-13b.

The third set of data shown in figures $4-13 a$ and $4-13 b$ is the experimental execution time on a single processor divided by $1,3,5,7$, and 9 processors. This overly simplistic model serves as a comparison model as it takes no overhead into account. In both figures the MPMW model overestimates for a single processor and underestimates after more than three processors are considered. The greatest concern is for the model prediction at the single processor. When using more than one processor, the model seems pessimistic but produces results similar to the actual execution times. The standard deviations of the experimental execution times range from two seconds to two minutes, though most runs using more than one processor have standard deviations of less than 20 seconds.

The MPMW model does not represent the execution of a distributed process very well according to figures 4-13a and 4-13b. The main reason why the difference between the two is so great is that the standard deviation when using a single processor is high, +1 one minute and 21 seconds in figure $4-13 a$ and $+/$ - one minute and 28 seconds in Figure 4-13b. This deviation is not shown in the figures but may emphasize the difference between the model and the actual execution time. 


\section{Number of Processors vs Run Time}

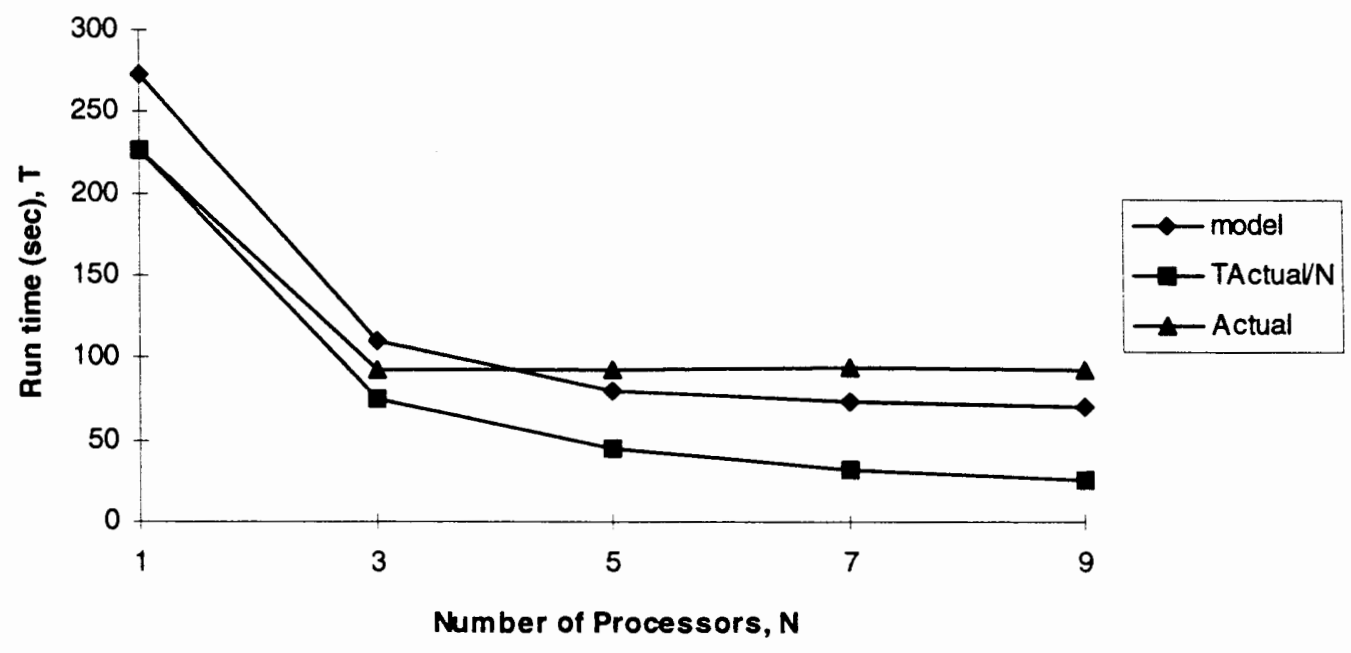

Figure 4-13a. 50x50 Matrices, G=20

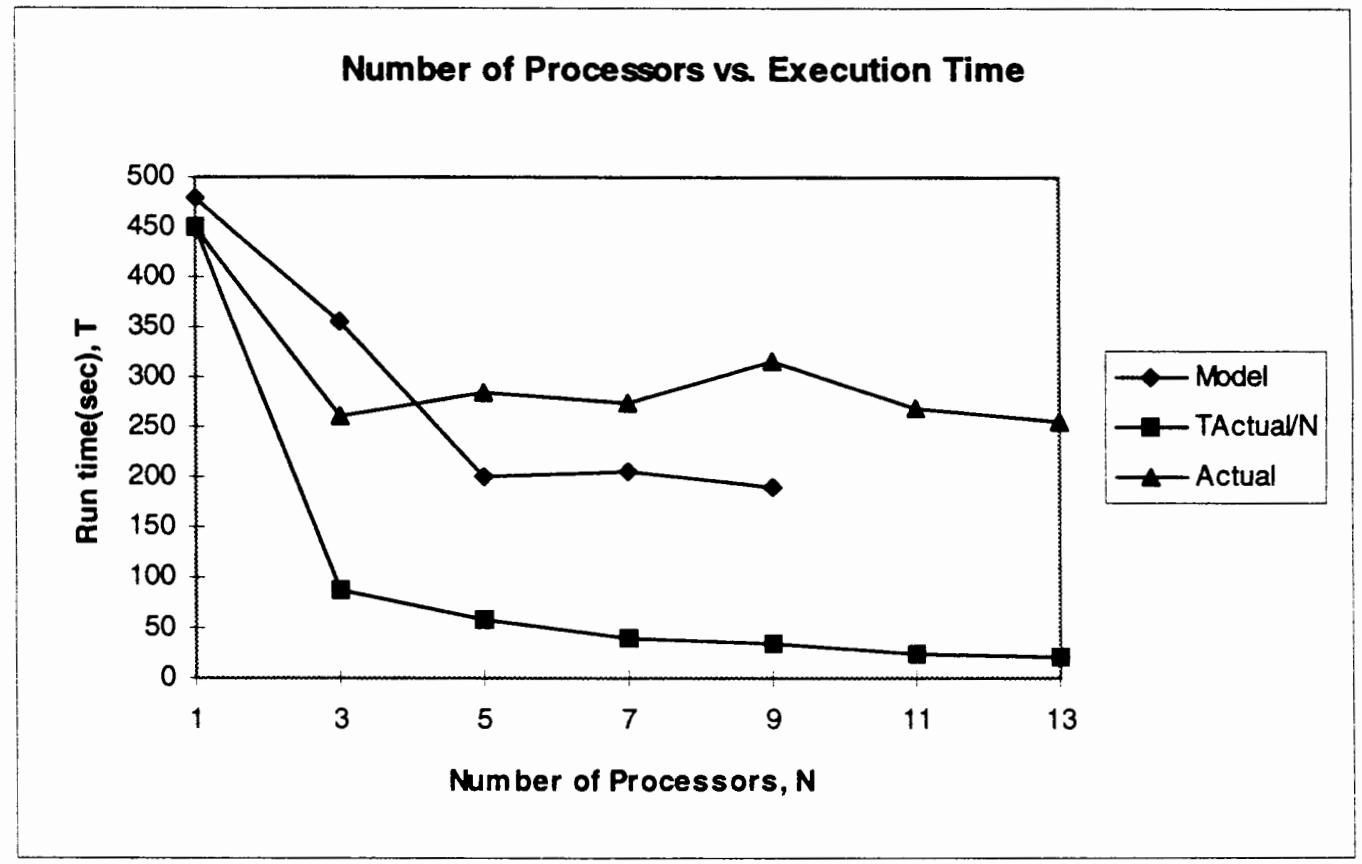

Figure 4-13b. $100 \times 100$ Matrices, $G=20$ 


\subsection{Phase 2 - Uneven Job Pools}

\subsubsection{System Measurements}

In this phase of the experimentation the LU decomposition tasks are separated from the back substitution tasks. Workers servicing the first pool perform an LU decomposition on the matrix found in their task file and place the result vector into the second pool. After retrieving tasks from the second pool, the worker performs a back substitution and places the final result in the final pool. This division of labor makes the job pools "uneven", as the LU decomposition is more computationally complex, $\mathrm{N}^{3}$ executions, than the back substitution, $\mathrm{N}^{2}$ executions. Thus the task computation time, the resource busy time and visit counts for each pool can be different. There are three interesting situations to examine, when the granularities are both one, when the granularity of the first pool is much greater than that of the second pool, and when the granularity of the second pool is much greater than that of the first pool.

In the first case, servicing the first pool takes much longer than servicing the second pool, but the migrating workers minimize performance loss from starvation in the second pool. The second situation will increase the severity of the starvation in the second pool and decrease the parallelism of the system. The third case may offer some performance gains since increasing the granularity of the second pool will balance the pools' workload. 
There are still 100 tasks overall and each task contains a 50x50 matrix to undergo first LU decomposition and then back substitution. Appendix B lists the specific experiments.

\subsubsection{Execution Times}

Figure 4-14 shows the real usefulness of migrating workers in the MWMP paradigm. Comparing the performance when the two pools are equal to the performance with uneven pools and a granularity of one shows very little difference. The migrating ability of the workers provide a buffer protecting system performance from variations in task sizes. Looking at the results when the granularity of pool A is 20 and the granularity of pool B is one and the results when the granularity of pool A is one and the granularity of pool B is 20 shows little overall decline in performance. Only when either of the granularities is 50 does the performance severely decrease. Thus while

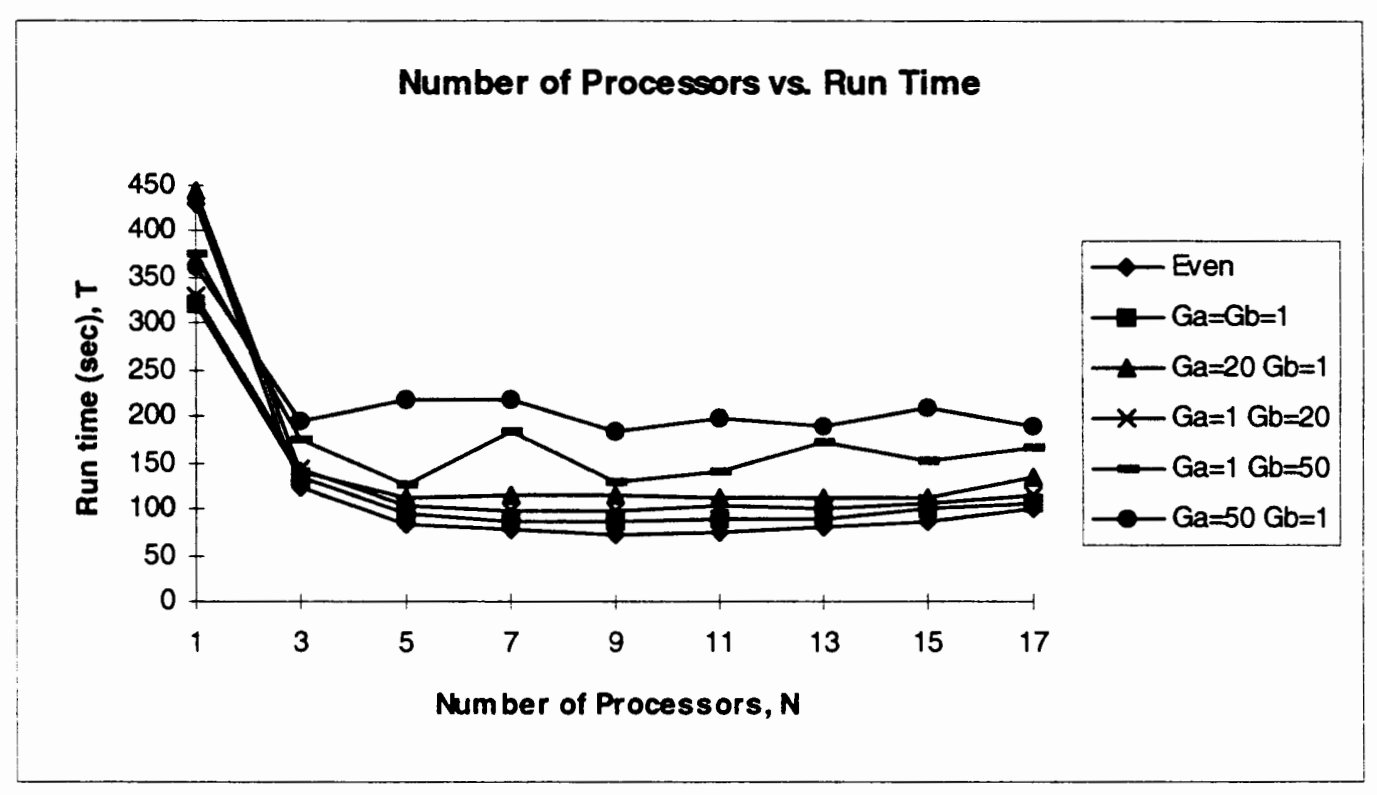

Figure 4-14. 50x50 Matrices 
there is an upper bound on the leeway provided by the migrating workers, they do offer quite a variation in possible bundle size. The important ramifications of this observation is that without even considering granularities, a system with migrating workers can handle pools with very different tasks sizes without the performance hit usually caused by starvation problems in this scenario.

Even with migrating workers, it was expected that increasing the granularity of the first pool would result in worse performance than increasing the granularity of the second pool, since the first pool contains the more time intensive tasks. There is a performance decrease, but not as dramatic as expected. This is due to the leeway provided by the migrating workers mentioned above. When the granularity of the first pool climbs to 50 , compared to one for the second pool, the expected increase in execution time is evident.

\subsubsection{Task Computation Time and te}

As discussed in 4.2.1.2 the average time it takes a worker to complete a bundle of tasks, te, should not change as more workers enter the system since this number reflects the task computation time only and includes no overhead. Figures $4-15 \mathrm{a}$ and $4-15 \mathrm{~b}$ show that this is indeed the case, the average computation times are independent of the number of processors. The variations seen in the times quite possibly reflect small differences in workers and system load. 
In Figure 4-15a, te $\mathrm{A}_{\mathrm{A}}$ is the task computation time since there is only one task per bundle. The te ${ }_{B}$ results, in Figure 4-15b, represent the computation of fifty tasks in each bundle. Note that pool A performs the LU decomposition while pool B does the back substitution, thus a single task in pool A takes approximately 20 times longer than a single task in pool B. Thus, having pool B bundle the tasks is a way to return some equivalence to the block of work done at a time in each pool. As shown in the results on "even" pools, having similar work loads at each pool is an advantage. Figures 4-15a and $4-15 b$ show that the computation time for the bundles at each pool are quite different, thus the granularity of pool B is too high to give equal pools. Experiments were also run with $G_{A}$ equal to one and $G_{B}$ equal to twenty but the te ${ }_{B}$ was still a factor of ten higher than $\mathrm{te}_{\mathrm{A}}$. To achieve optimal performance a user may run more tests to determine the best granularity balance.

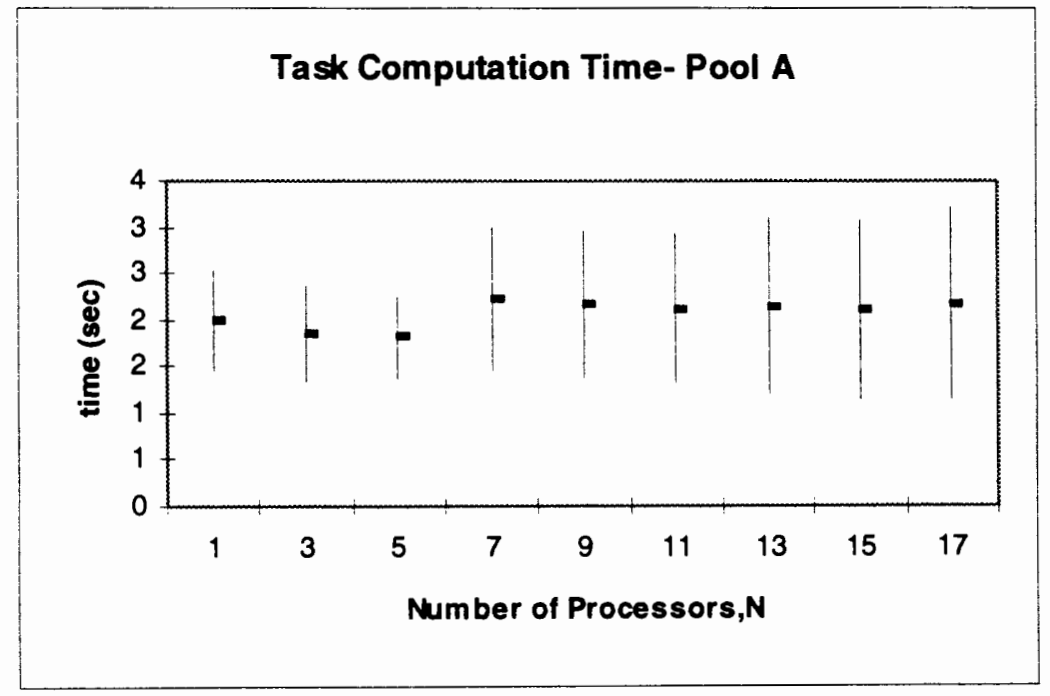

Figure 4-15a. Uneven Pools, $\mathrm{G}_{\mathrm{A}}=1$ 


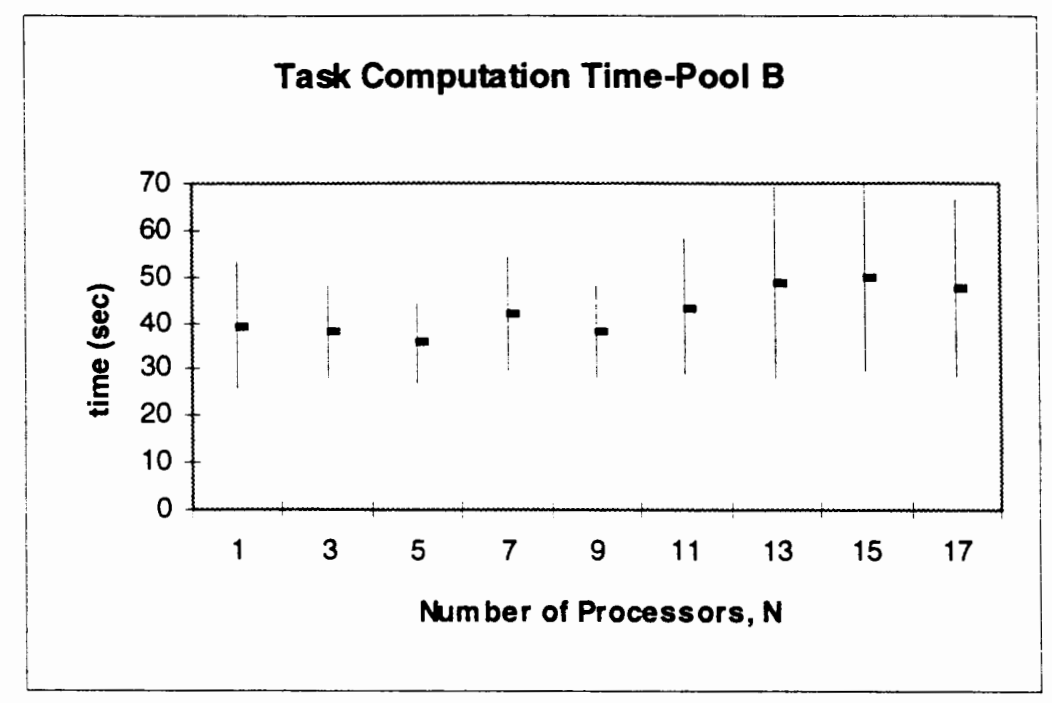

Figure 4-15b. Uneven Pools, $\mathrm{G}_{\mathrm{B}}=50$

\subsubsection{Resource Busy Time}

As with task computation time, resource busy time should be examined on a per job pool basis. Figures $4-16 a$ and $4-16 b$ show the trend in $B_{A}$ and $B_{B}$ when the granularity of pool A is one and the granularity of pool B is fifty. Similar results occur when $G_{A}=1$ and $G_{B}=20$. The results from the even pools in Section 4.2.1.3 present a nearly linear increase in resource busy time as the number of processors increase.

As the number of workers in the system increase, the job pools (the resources) are more likely to be busy with a worker at any given point in time, since there are more workers seeking to service a specific job pool. Thus the resource busy time increases. The large resource busy time values are caused by only a few workers, however. Looking at the median resource busy time, the overall increase is still seen, though it is much less 


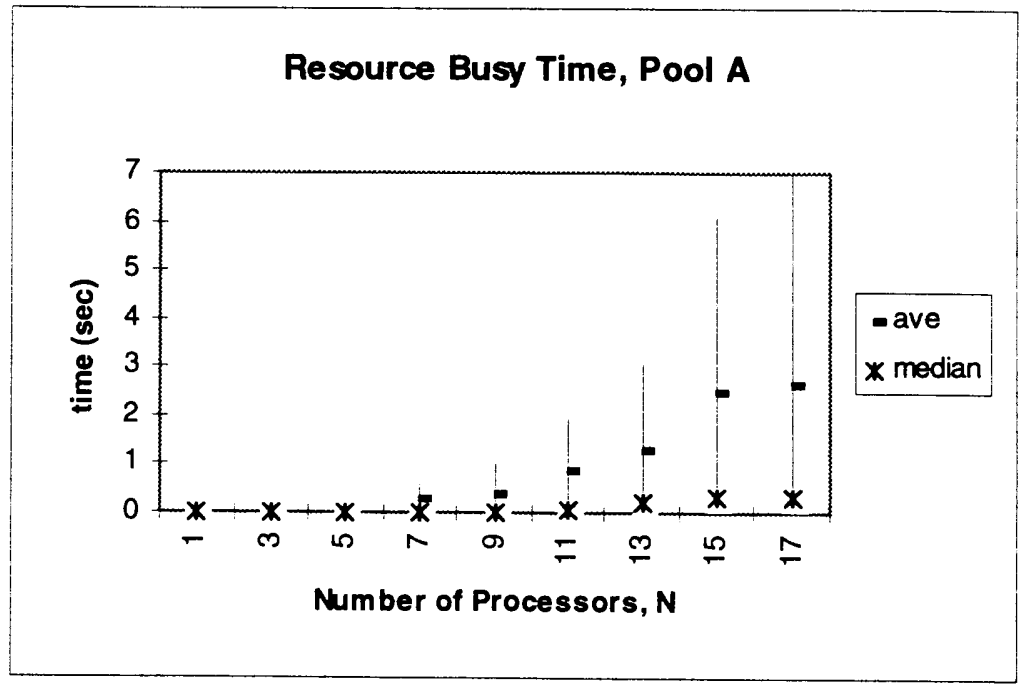

Figure 4-16a. Uneven Pools, $\mathrm{G}_{\mathrm{A}}=1$

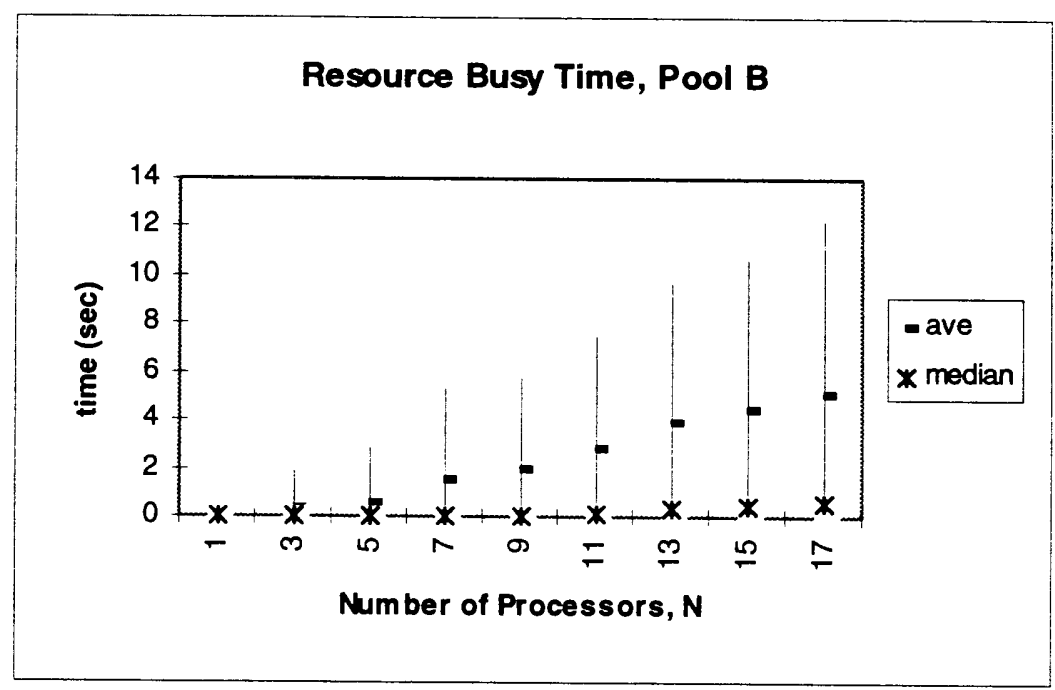

Figure 4-16b. Uneven Pools, $G_{B}=50$

dramatic. Thus, there is a greater likelihood that a worker will run up a large resource busy time when more workers are present, but this does not affect all the workers in the system all the time. 
It should also be noted that the resource busy time is a function of the number of tasks in a bundle. For example, the resource busy times for pool A are about half of those for pool B. Pool B is handling bundles of fifty tasks, verses the bundles with one task from pool A. Since resource busy time includes the time to transfer the task files to a worker's directory the source of the extra time is clear.

Reversing the bundles so that pool A has bundles of fifty tasks and pool B has bundles containing a single task produces similar, though less clear results. Figure 4-17a and 4$17 \mathrm{~b}$ show the resource busy time as the number of processors increase under this configuration.

In general, the resource busy time does increase with the number of processors, though we begin to see the peak at fifteen processors, then a decline in the time required in the spinlock results in the even pools (4.2.1.2) and later in (4.3.1.4). With bundle sizes of

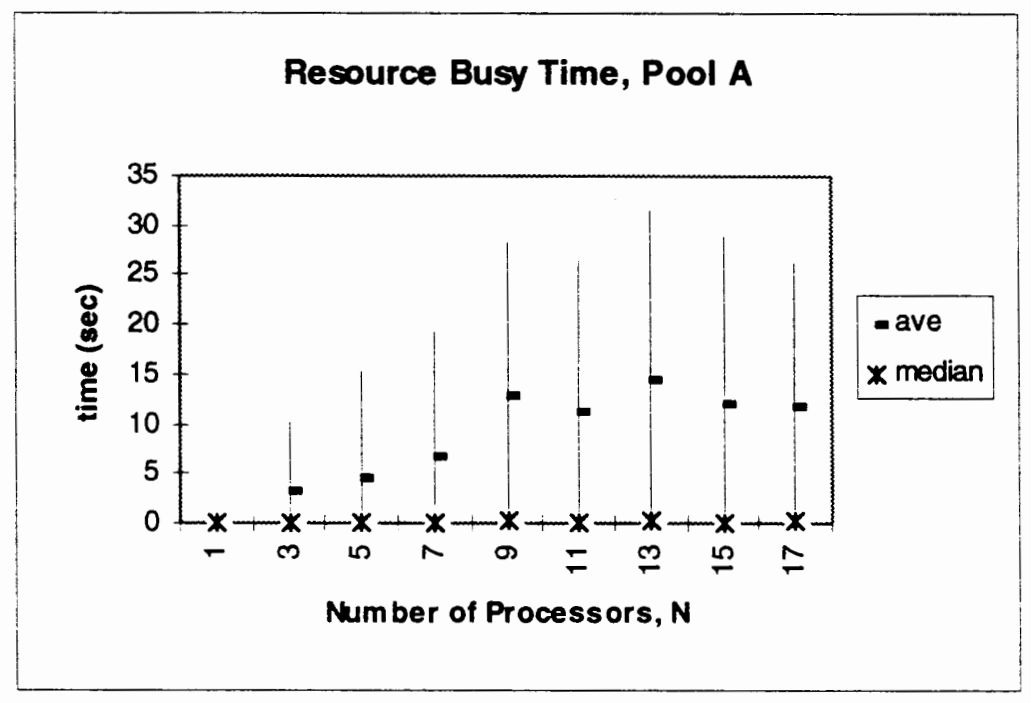

Figure 4-17a. Uneven Pools, $G_{A}=50$ 


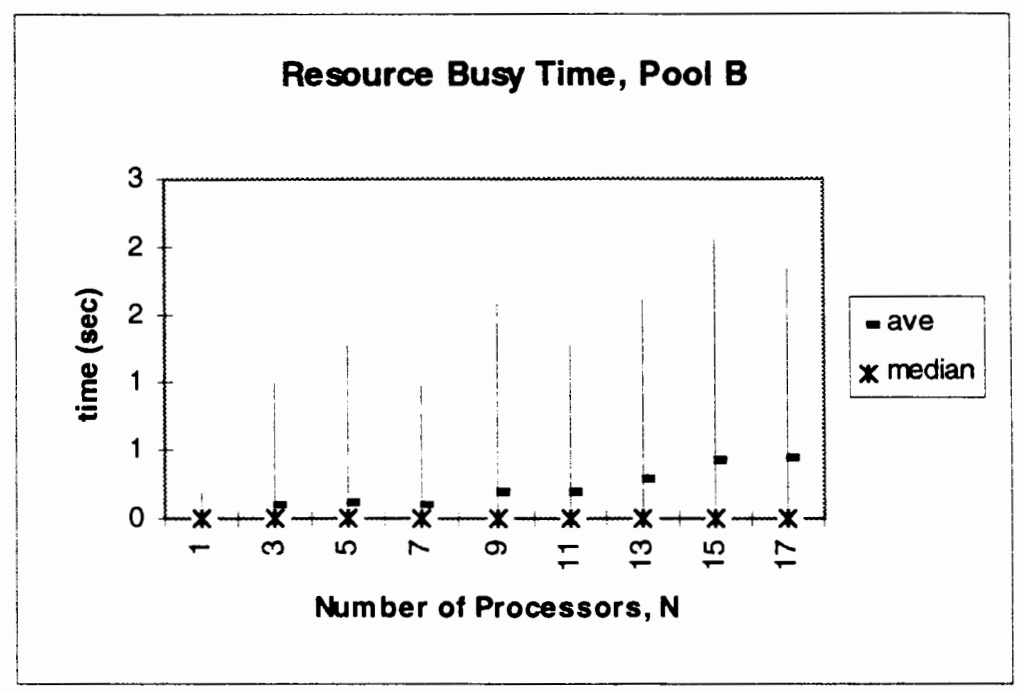

Figure 4-17b. Uneven Pools, $\mathrm{G}_{\mathrm{B}}=1$

one task, the second pool will be able to accept service from many more workers that pool A, since A only contains enough bundles for two workers. After the two bundles are transferred out, the workers just wait in line to look in and see that no work is present.

After checking pool A once and finding it empty, a worker will stay at pool B until the program terminates. Thus the resource busy time in Figure 4-17a is based on two quantities, the time it takes to get the two bundles of fifty tasks and the time it takes for each worker to check and see that pool $\mathrm{A}$ is empty. This helps explain the deviation from the long time average, while a single worker is accessing the pool and retrieving one of the two bundles, the resource busy time will be a single high count. As multiple workers gain access and find the pool empty, shorter stays at the job pool are seen and 
the resource busy time accounts for the accumulation of many shorter visits to the job pool.

Figure $4-17 b$ is a plot of the scenario where many workers can get work from pool B, since 100 individual bundles will pass through it. Thus the resource busy time accounts for multiple visits to retrieve a single task file at a time. These visits will take longer than empty pool visits, but shorter than moving fifty files from one job pool to another. Since fifty tasks at a time are being dropped into pool B, few workers will see an empty pool, until all the tasks are done, at which time the workers exit. The fewer the workers in the system the less the likelihood that a worker will see pool B empty until all tasks are finished. This phenomenon explains why the resource busy time for pool B is higher at the lower worker numbers, and climbs relatively little as the number of workers increase. This is not seen in the first case where pool A has a granularity of one because of the way the pools are constructed. Pool A receive no new tasks, while pool B receives tasks until pool A is empty. Thus pool A is only accessed by workers retrieving tasks, Pool B must be accessed to receive the finished tasks from pool A as well as for the retrieval of tasks from workers servicing it. Thus the resource busy time is higher for pool B since it must be accessed twice for each task.

Comparing the median and the averages in Figures 4-17a and 4-17b, a large difference is seen. Most data points have relatively low values though a few points have very high values. These points are not outliers and cannot be ignored because there are data 
points scattered throughout the range. Thus the average is artificially high. The median helps illustrate this. In general, the median line follows the behavior of the average line, but the median values are much less. This emphasizes that most of the data points are located in the lower values of resource busy time. Thus the above arguments relating pool behavior to resource busy time are still valid, though the total effects are not as severe as the average indicates.

\subsubsection{Spinlocks}

Unlike the resource busy time, the spinlock results are not similar to the results from the even pools experiments. To clarify the examination, the number of spinlocks were separated into three types: those gaining a lock to get tasks from pool A, those placing tasks in pool B, and those getting tasks from pool B, see Figures 4-18a through 4-18c for the results when pool A has a granularity of one and pool B has a granularity of fifty.

The large variation in these results is misleading, only a very few spinlocks counts are near the maximum, most range from 1 to 30 . The median emphasizes this point. These few (less than thirty out of thousand data points) spinlock values are over a thousand, artificially driving up both the spinlocks and the resource busy time results. These instances are possibly caused by exclusion of workers by the lock master or some other unseen phenomenon. If a worker attempted to gain a lock on an already locked pool, it increments its spinlock and attempts to gain a lock again. Since the lock master only 
serves one worker at a time, a different worker then calls the lock master attempting a lock on that pool. If the pool's lock is released at that point, then the second worker's attempt is successful and it gets the lock. Now the first worker successfully calls the lock master and finds the pool still locked. It is possible that this unordered access scenario can occur multiple times, excluding the worker and driving up its value of Vs. The potential bottleneck caused by the lock master could be the driving force behind this, as it serves the lock request of one worker at a time, so not only is a worker attempting to get the pool during an unlocked period, it must wait to get access to the lock master as well. Redesigning the lock master, or the locking mechanism, for a more ordered access could relieve this problem.

Once the above inconsistencies are taken into account, the unequal job pool

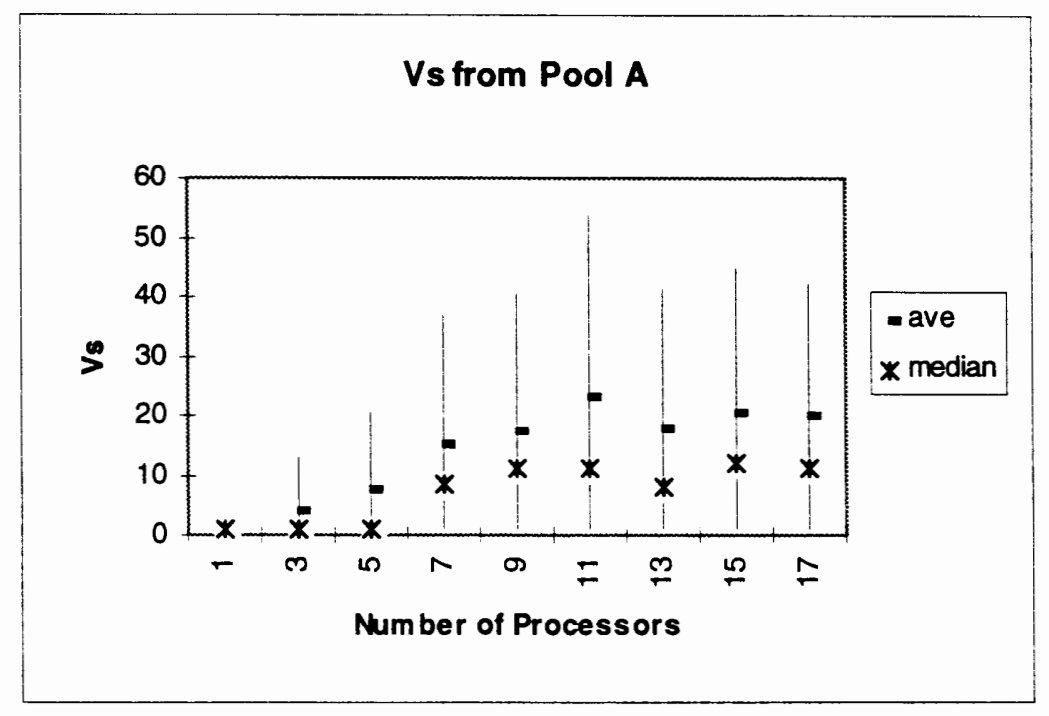

Figure 4-18a. Spinlocks to Get Tasks from Pool $A, G_{A}=1$ 


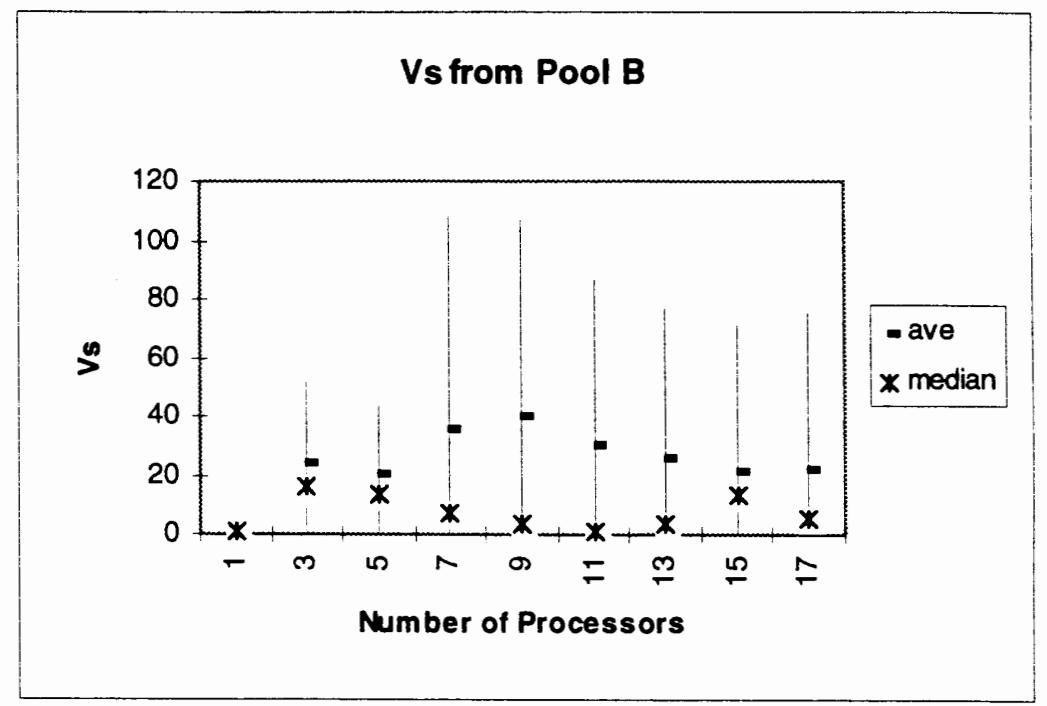

Figure 4-18b. Spinlocks to Put Tasks into Pool $B, G_{B}=50$

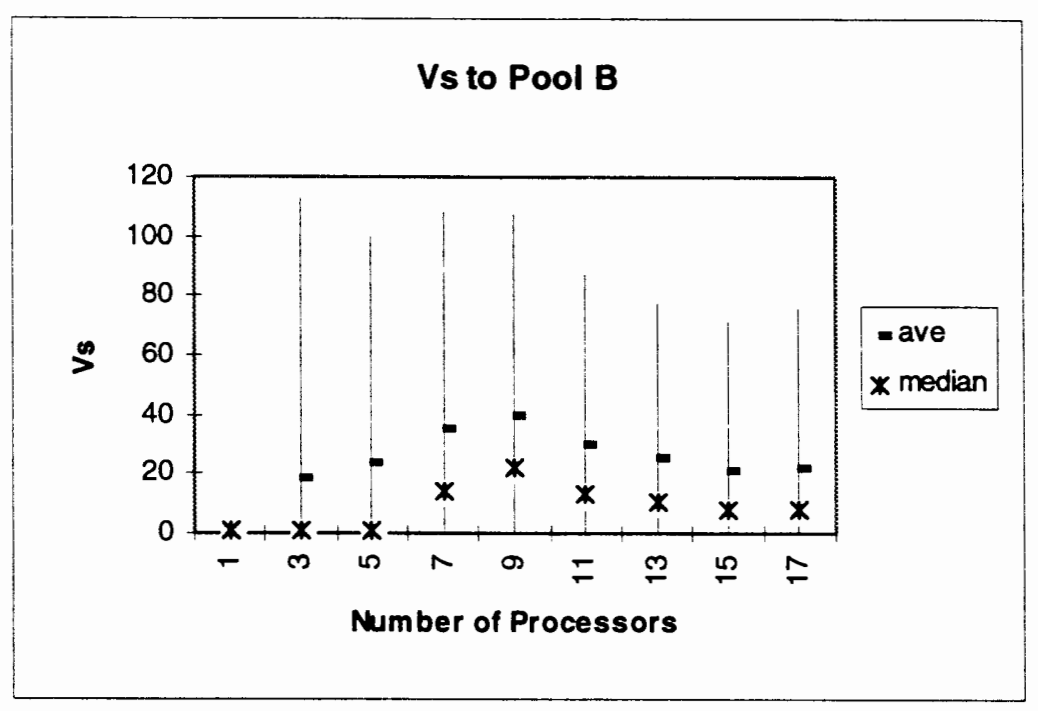

Figure 4-18c. Spinlocks to Get Tasks from Pool B, $\mathrm{G}_{B}=50$

configuration and worker migration easily account for the results in Figures 4-18a - 4-

18c. The results in Figure 4-18a are similar to those results seen in the even pools results. The spinlock count increases to a peak then decreases as the number of workers in the system continue to increase. As the granularity of pool A is one task per bundle 
and the system starts with one hundred bundles in job pool A, this result is not surprising, at this point the system looks exactly like the even pool system. Also, the worker will work exclusively on pool A until 70 tasks ( $70 \%$ of 100 tasks) have accumulated in pool $\mathrm{B}$, then one worker will leave and the remaining workers will continue servicing pool A until it is empty. At that time one more worker will service pool B, emptying it, and the rest of the workers will exit the system.

In Figure 4-18b the spinlock values seem to increase quickly, then slowly decline as the number of workers in the system increases. Since only two bundles exist in pool B, only two visits to pool B per program execution will occur. The rest of the visits will be workers servicing pool A depositing their finished work into pool B. Therefore results similar to the even pool experiments are seen. At first glance, the spinlock values for getting bundles from pool B as the number of processors increase seems very odd. It must be noted however, that only twice during program execution do workers get a bundle from pool B. This means that very few data points are used in figure 4-18c making the results looked skewed.

It is informative to compare the above results with the results from an experiment where the granularity of pool A is fifty and the granularity of pool B is one. This swapping of granularities really shows how the two pools differ and how migrating workers react.

Figures 4-19a - 4-19c are given for such comparison. 
As with the spinlock results from the even pools in Section 4.2.1.4 and Figure 4-1 lb, the majority of the data points are in the lower values, but a few extremely high outliers drive up the deviation. The median behavior follows the behavior of the average, while illustrating that the majority of the data points are close to one. As mentioned in Section 4.2.1.4 these high valued outliers are most probably workers whose first attempt at the lock is immediately after another worker gains that lock. In cases where the granularity is fifty, that worker will continue to spinlock, finding a locked pool, until all fifty files are transferred to the workers directory and the appropriate files updated. Should the worker lose that lock opportunity to yet another worker, it can potentially spinlock while all 100 files are moved and files updated. This would account for the few very large spinlock counts. The average measured time to attempt a lock can be estimated at 7.5 milliseconds. The time to transfer 50 files ( $50 \times 50$ matrices) is measured using the UNIX "time" command to yield an estimate of $11+/-1$ seconds. Thus in the time it takes a worker to transfer 50 task files, a worker can conceivably have attempted a lock over 1400 times. Indeed some of the spinlock counts are over 1000, illustrating this worker exclusion. This makes the standard deviation very high.

From the MPMW paradigm and Section 4.2.1.4 the spinlocks for a pool with a granularity of fifty could be expected to rise until $N$ equals two processors, then decrease again. While two processors were not a test point in the experimentation, a general trend of rising toward a peak at three processors, then decreasing as the number 
of processors continue to rise, see figures $4-19 \mathrm{a}$ and $4-19 \mathrm{~b}$. This supports the expected results, though not as clearly as the case with even pools. Also adding to the deviation of figure 4-19a and 4-19c is the small data set. Since pool A has a granularity of fifty, only two workers successfully access the pool per program execution. Each experiment was repeated five times at each number of processors, giving a total of ten data points

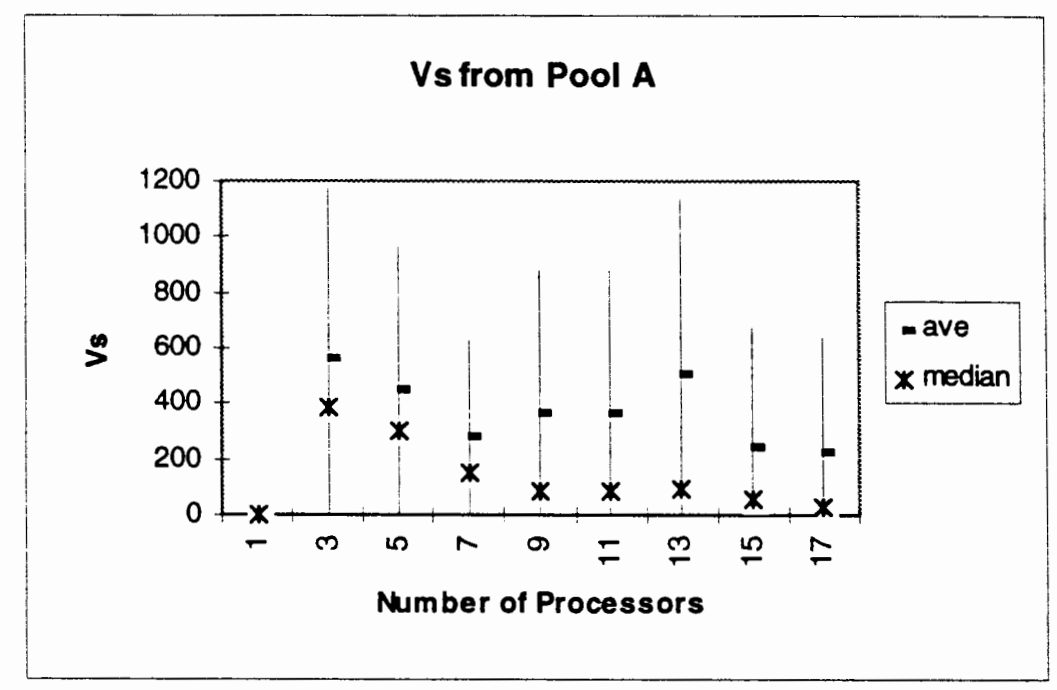

Figure 4-19a. Spinlocks to Get Tasks from Pool $A, G_{A}=50$

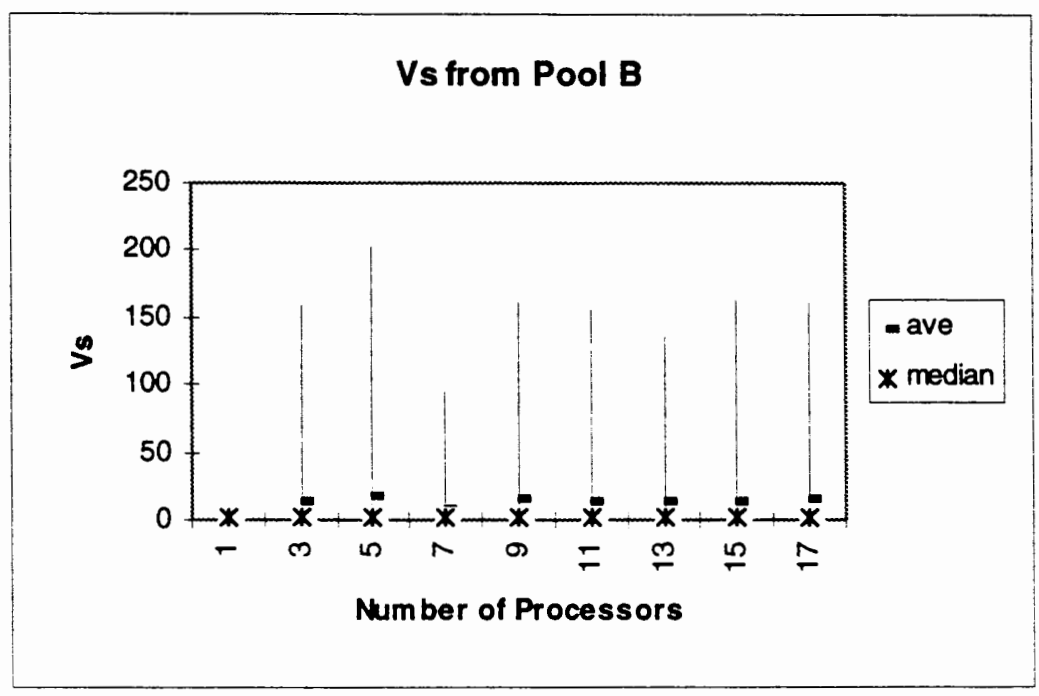

Figure 4-19b. Spinlocks to Put Tasks into Pool B, $\mathrm{G}_{\mathrm{B}}=1$ 


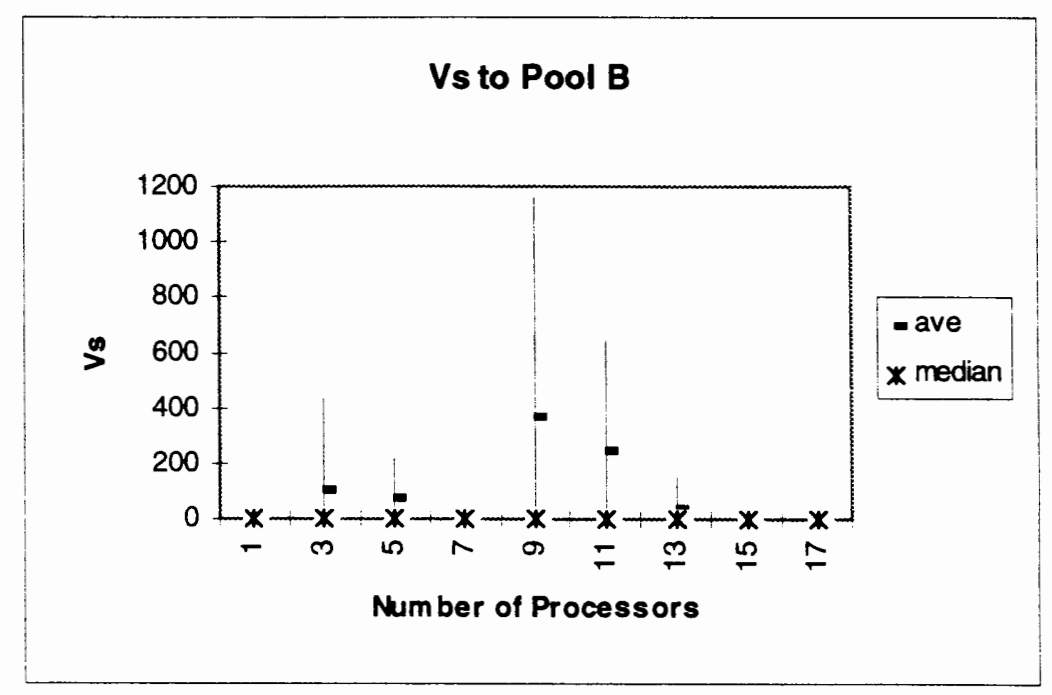

Figure 4-19c. Spinlocks to Get Tasks from Pool B, $G_{B}=1$

getting bundles from pool A and depositing them into pool B. Since pool B has a granularity of one, there are 100 data points per execution, or 500 per experiment (at a given number of processors). Thus, the data outliers have much less affect on results concerning pools with low granularities.

As with the results in figures 4-18a-4-19c, the way the workers migrate affects the outcome. Once the two bundles at pool A are taken by two workers, the rest of the workers (if any) in the system check pool A, find it empty and go on to pool B. While waiting for one of the two " $\mathrm{A}$ " workers to deposit there tasks, the rest of the workers continually access pool B looking for tasks. When the first "A" worker releases its fifty finished workers into pool B then the workers all become busy, as there are more than enough bundles for all. That time of continually checking pool B for work is idle time for those workers. 
The above situation illustrates the real effects of the mis-matched pools. Pool A, in the second case, has the more computationally intensive task and a granularity of fifty, meaning only two workers can service the pool and all 100 tasks, while additional workers are useless and wasteful. Once the bundle of fifty completed tasks are placed into pool B where the granularity is one, then all workers remain active. On the other hand when pool A has a granularity of one and contains the computationally expensive tasks, all the workers can service pool A until enough tasks are transferred to pool B for some workers to migrate to pool B to prevent a backlog. In this scenario pool B has easier tasks, but they are bundles into sets of fifty. In this case, all workers stay at pool A until there are seventy tasks in pool B, then a single worker can successfully service pool B, taking a bundle of fifty tasks. After the second fifty tasks are completed another worker will take that bundle of fifty and the rest of the workers will exit rather than remain idle. Therefore, it is important that when there is a pool with fewer bundles than the number of workers in the system, it should be at the end of the chain of job pool, so that unused workers exit and become free rather than remain idle. Also, the effects of problem size are seen. If a larger problem exists upstream, its bundle size should be small enough so that all workers are active until tasks start to show up in the downstream pool.

\subsubsection{Experimental Data vs. Models}

Using the system measurements, the predictive abilities of the MPMW model can be examined. The uneven pools in this phase represent exactly what this paradigm and 
model were designed for. In most realistic cases the work load, whether decided by task size or bundle size, will not be evenly distributed among the work loads, the computation that must be done within a single pool may exceed that of a subsequent or predecessor pool. This paradigm, and its associated model, deal with the uneven pools through bundles and migrating workers to give good results. For this discussion two experiments are used: when $\mathrm{G}_{A}=1$ and $\mathrm{G}_{B}=50$ and when $\mathrm{G}_{A}=50$ and $\mathrm{G}_{B}=1$.

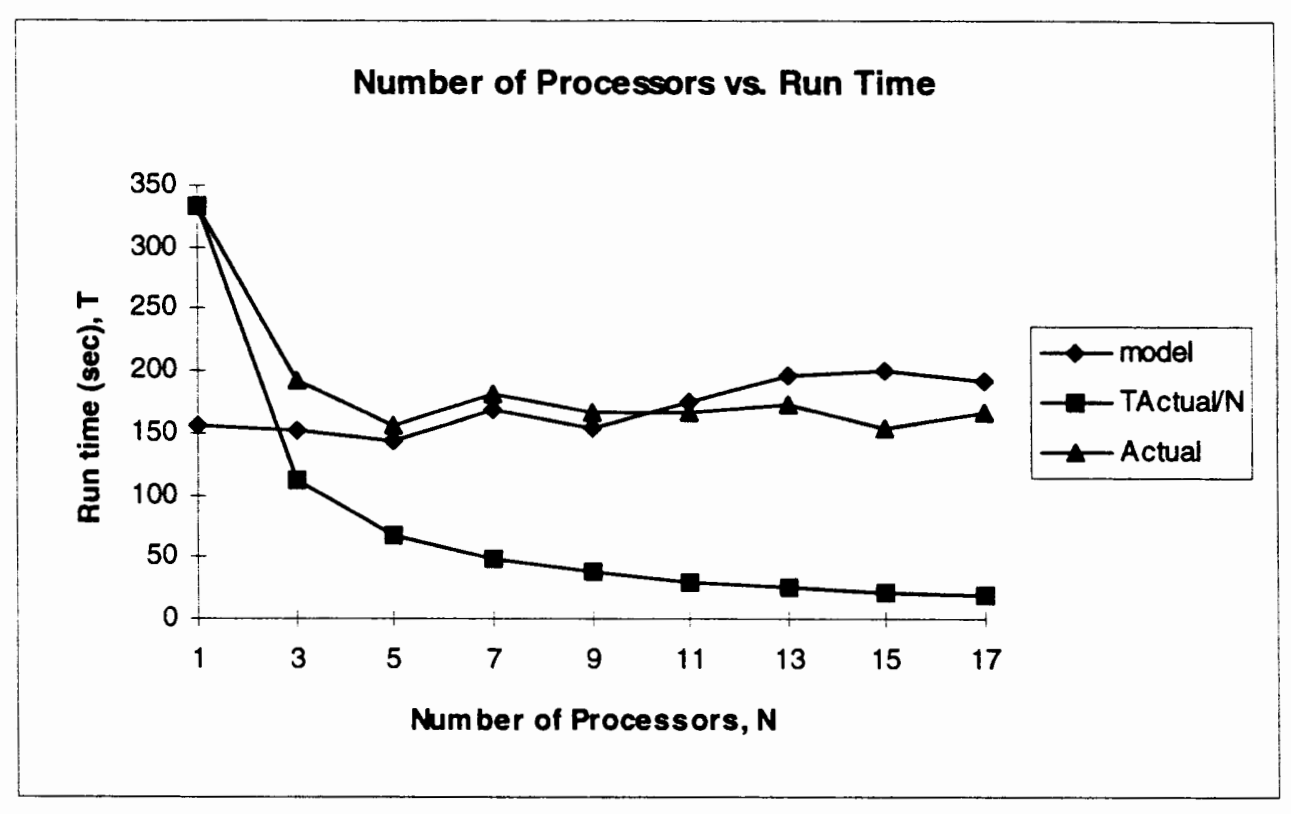

Figure 4-20. Comparing Model verses Actual Data, $G_{A}=1$ and $G_{B}=50$

It is more difficult to find the optimal number of processors intuitively when the job pools contain work of differing complexity. One job pool doles out 100 bundles, indicating a large number of workers would be useful, while the other pool contains only two bundles for two workers. 
As mentioned in the last section, if the pool with two bundles is first, then the remaining workers are idle, while if this pool is last, excess workers exit. Applying the above system measurements to the MPMW model when $G_{A}=1$ and $G_{B}=50$, the model line in figure 4-20 results. According to this model, using more than five processors gains nothing. Even though the first pool has 100 bundles, potentially for 100 workers, the associated overhead for this size task makes more than five processors useless. The experimental data gathered agrees with the model's assessment. The MPMW model is designed for distributed systems, thus it is not an adequate model for the serial case. In

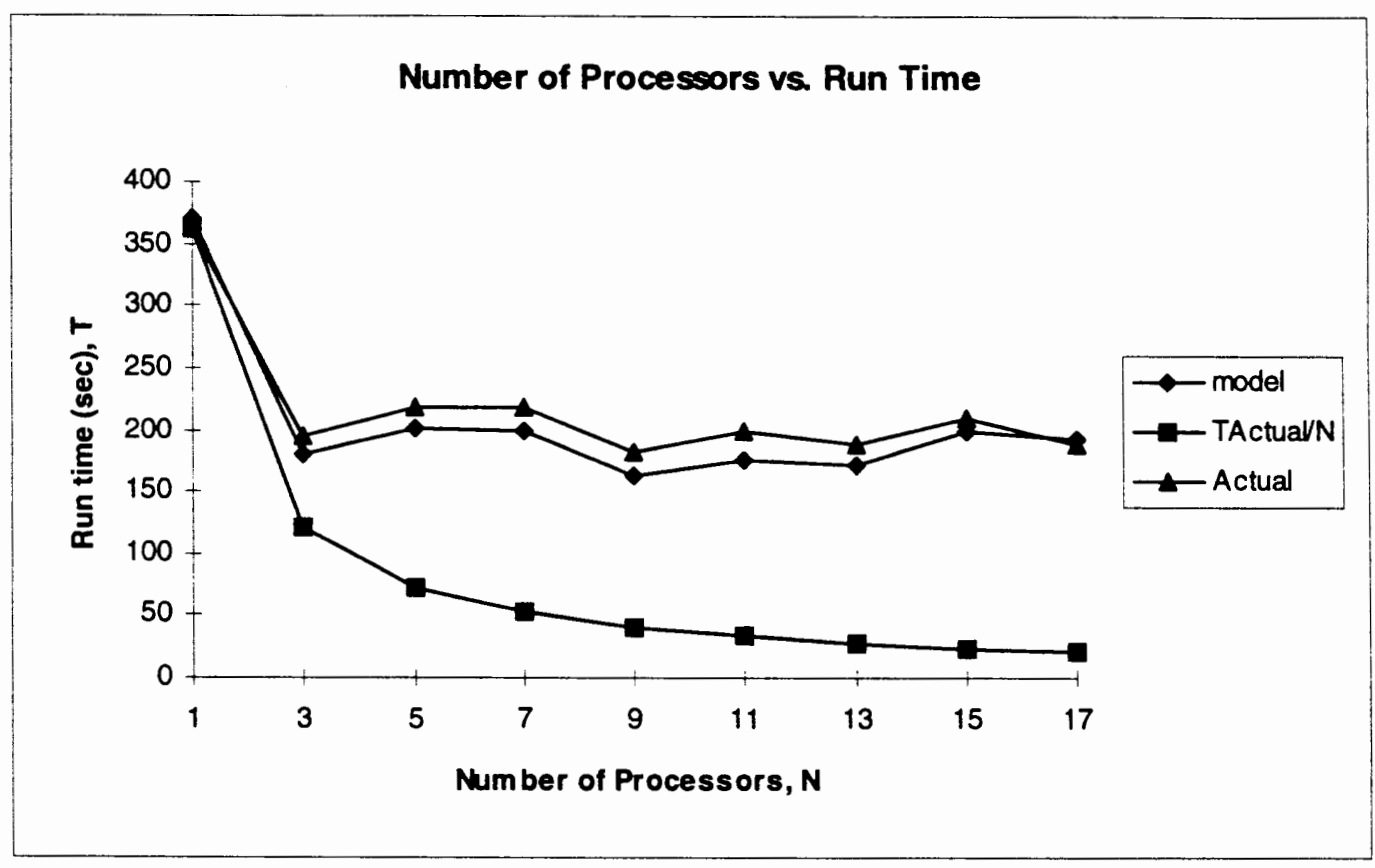

Figure 4-21. Comparing Model verses Actual Data, $G_{A}=50$ and $G_{B}=1$ general, the model predicts the general behavior of the experimental results, see figure 4-20. Uncontrollable variables, including locally varying processor loads, contribute to the differences between the model and the actual data. The average percent difference 
between the model and actual data is $13 \%$, excluding the data for $\mathrm{N}=1$. Figure 4-21 shows the corresponding results when $G_{A}=50$ and $G_{B}=1$ for 100 tasks. This is the worst case scenario for the uneven pools, as all workers past the first two must wait for the first bundle of fifty to be completed and deposited into job pool B before they do useful work. The model predicts that more than three processors is essentially useless; the experimental data concurs. In this case the average percent difference between the model and the actual data is $7.5 \%$. In general the model gives more accurate predictions of the behavior of uneven pools than of perfectly even ones. Since the model was designed for the non-balanced nature of the uneven pools, this result is encouraging.

The MPMW model was developed for multiple processor cases, and as seen in Figure 420 , it does not predict well for the $\mathrm{N}=1$ case. The model derivation is based on two pools being serviced by workers concurrently, a scenerio that does not occur at $\mathrm{N}=1$. However, looking at the results from Figure 4-20 and 4-21, the model does a better job of predicting the behavior of a single processor when the first pool characterizes the work. In Figure 4-21, the first pool has bundles of fifty tasks, whereas the second pool has one task per bundle, thus the first pool characterizes the system. In Figure 4-20, the second pool has a much higher granularity and therefore characterizes the system. Thus the model does a poor job of predicting run time at $\mathrm{N}=1$ in that case.

The simple $T_{\text {Actual }} / \mathrm{N}$ model is shown for comparison purposes. The $T_{\text {Actual }} / \mathrm{N}$ data emphasizes the increase in overhead between even and uneven pools. Looking at 4-13a 
and 4-13b and comparing those results to figures $4-20$ and $4-21$, the overhead cost is quite apparent. In the even pools, the experimental data seems to follow the general behavior of the $\mathrm{T}_{\mathrm{Actua}} / \mathrm{N}$ data, though with higher times. In the uneven pools, the divergence of the $T_{\text {Actual }} / \mathrm{N}$ model and the actual data is readily apparent. The MPMW model exhibits no such divergence, and gives better overall results.

An important observation can be made by looking at Figures 4-14, 4-20, and 4-21; the MPMW model can predict the general behavior of the system as the granularity is changed. The MPMW model can not pinpoint the optimal granularity for a system, without extensive experimentation. It can, however, predict whether a larger or a smaller granularity will improve system performance. 


\section{Chapter 5}

\section{Conclusions and Future Work}

\subsection{Conclusions}

The purpose of this thesis is two-fold, first providing a methodology for distributed computing and second developing a model of that methodology. The methodology developed in this thesis, the MPMW paradigm, is presented to be an easy to use, quick and efficient way of implementing problems into distributed computing solutions. First to allow easy breakup with little parallel programming knowledge, second to allow efficient use of NOW's to produce the best possible results while minimizing the effects on other users. The model is meant to not only describe the system but also to give the user predictive ability in the estimation of resources required (processor time). Also the model provides an excellent way of judging the cost and gains of bundle size, task size, number of processors and effects of system usage.

Task size and bundle size affect performance in distributed systems; the MPMW paradigm presented in this thesis predicts this influence. However, the impact of nonoptimal task sizes is minimized with migrating workers and job pool bundles. Migrating workers provide a cushion or buffer against the differences in task sizes in multiple pools. Figure 4-14 shows the buffering provided by the migrating workers by comparing wall clock time for job a with same size tasks in two pools to jobs with uneven task sizes in the pools. The graph also reflects bundle size, an easy way to 
change the apparent task size of the system. The even task size system provided the best wall clock time, but the uneven task size system had a relatively small performance hit when the granularities of the two pools were both one. Even when the granularity was skewed to one pool or another by twenty (one pool had twenty, the other one), the performance hit is minimal. Increasing the granularity of one pool to fifty and leaving the other at one starts to overrun the buffer, decreasing performance noticeably. The task sizes were initially different, an LU decomposition verses a back substitution, but increasing the granularity of either pool to fifty effectively increases one task size fifty times over the other.

The granularity can be a powerful tool to a user of distributed computing, especially with the MPMW paradigm. The data in the even pool experimentation shows that increasing the number of tasks per bundle for that particular task size gains no performance benefits. However, the uneven pools experiment phase shows the potential benefits of grouping many small tasks in a bundle in one pool, while leaving larger tasks in small bundles in another pool. The performance gain from using bundled tasks in the LU experimentation is not obvious, but a situation can be envisioned where several small tasks can be grouped to form a pool full of "supertasks" that are of equivalent size to tasks in the other pool. Thus even pools are created increasing performance. In this case the difference between the task sizes was not great enough to show spectacular results. 
An important, if unexpected, observation from the experimentation is that the number of bundles in an upstream pool should equal or exceed the number of workers in the system. If there are more workers than bundles, workers sit idle wasting processor time. If such pools can be placed as the last pool then unused workers exit when all the bundles are taken. If this placement is not possible then bundles should be divided to equal the number of workers for maximum efficiency.

The model developed to predict behavior of a MPMW system can provide an estimate of needed wall clock time given certain resources and allows the user to alter bundle size, task size, and the number of number of processors to reflect system usage conditions or time constraints. The model is designed to predict the performance of migrating workers and multiple pools (specifically two) in the system. Therefore, the model's predictions for single processor performance are not usable. Overall, the model's predictions follow system behavior, though individual measurement points have been as much as $30 \%$ off of the experimental data. Most predictions are less than $15 \%$ off of the experimental data. The model is also adequate for predicting the general behavior of the system as granularity changes. 


\subsection{Future Work}

The MPMW paradigm and model spark many exciting avenues of potential work. There are issues dealing with worker migration, fault tolerance, pool dynamics, and system modeling. Most of these issues would improve the usability and accuracy of the model and the paradigm itself.

Currently, the decision by a worker to migrate depends on the percentage of tasks left in one pool compared to another. If a worker has been serving pool A, and pool A has more than $30 \%$ of the total tasks, then the worker will continue to service that pool. If less than $30 \%$ are found in pool A the worker moves to pool B. The decision to make the cutoff $30 \%$ is largely arbitrary. For each system, there is potentially an "optimal" cutoff, some equation could be developed with further study that could determine this cutoff, perhaps even dynamically at runtime.

Like the decision to migrate, the decision on bundle size could be controlled by an equation or model. The task size is largely decided by the dynamics and logistics of the program being split into tasks. For the most part, this limits the ability of the user to control task size. The ability to bundle tasks together regains some control for the user. A study on optimal work amounts would be extremely interesting. Finding the optimal amount of work to put in a task for the best overall performance would allow users to use bundling (and granularity) as a tool to attain optimal task size. 
While the MPMW paradigm is robust, it has no specific fault tolerance measures. Applying a fault tolerance system to the MPMW pool would be fairly simple. Strumpen [1] uses what he calls a workpool for dynamic load balancing and fault tolerance. Each task in the work pool is tracked until it is completed, thus if a machine fails, the task is still in the workpool and can be retrieved and completed by another worker. A fault tolerance system similar to this could be easily implemented to provide robustness to the MPMW paradigm.

This thesis addresses the MPMW paradigm in terms of two job pools in sequence. This paradigm and the model are both applicable to systems with many more pools. Not only does the paradigm apply to many job pools, it can be applied, with little modification, to systems with forking pools, joining pools, and any combination of the primitives in figure 2-2. Each node on a task graph could become a job pool in a large system with potentially hundreds of task types (job pools) and control threads. The MPMW paradigm is designed to be applied to these types of jobs. Thus this work is ready to be applied to complex jobs showing its real functionality.

Finally, there are many areas of improvement and refinement open on the model itself. For example, the $\mathrm{Q}(\mathrm{N}-1)$ term can be estimated easily by $\alpha$ in most cases, eliminating a fairly complex equation from a practical form of the model. Currently, the system 
measurements, B, Vs, and te, are measured in the system and used in the model. A way to determine these values for all cases given a few simple tests should be run. This is the most cumbersome part of using the model. From the results in this thesis, it seems that equations describing the behavior of B and Vs could be developed from simpler system tests. The would increase the predictive strength of the model and remove some deviation from the calculation. As mentioned previously, this model provides good system estimates, but the error is apparent, thus more work on the model could improve it. In this thesis the model is left as is, since it is roughly predictive and very descriptive of the MPMW paradigm. 


\section{References}

[1] Strumpen, V. Coupling Hundreds of Workstations for Parallel Molecular Sequence Analysis. Software - Practice and Experience. 25, 3(March 1995), 291- 304.

[2] Nibhanupudi, M.V., Norton, C.D., and Szymanski, B.K. Plasma Simulation on Networks of Workstations using the Bulk Synchronous Parallel Model. Int'l Conf. Parallel \& Distrib. Proc. Tech. and Applications, Athens, GA., (November 1995).

[3] Schaefer, Linda Analysis of a Coordination Framework for Mapping CoarseGrain Applications to Distributed Systems. Thesis, Portland State University, 1991.

[4] Zhang, X. and Yan, Y. Modeling and Characterizing Parallel Computing Performance on Heterogeneous Networks of Workstations. 7th IEEE Symposium on Parallel and Distr. Computing, San Antonio, Tx., (October 1995), 25-28.

[5] Zhang, X., Yan, Y. A Framework of Performance Prediction of Parallel Computing on Nondedicated Heterogeneous NOW. Proc. of 1995 Int. Conf. of Parallel Processing, CRC Press, Vol. 1, (August 1995).

[6] Anderson, T.E., Culler, Patterson. A Case for NOW Networks of Workstations). IEEE Micro 15, 1(February 1995), 54-64. 
[7] Koester, D.P. SuperComputing 94 - Networking Technologies Summary. NPAC Technical Report - SCCS 708. (November 1994).

[8] Kleinrock, L. and Korfhage, W. Collecting Unused Capacity: An Analysis of Transient Distributed Systems. IEEE Trans. Parallel Distribut. Systems.. 4, 5(May 1993), 535-546..

[9] Cap, H.C. and Strumpen, V. Efficient Parallel Computing in Distributed Workstation Environments. Parallel Computing 19, (1993), 1221-1234.

[10] Celenk, M. and Wang, Y. Parallel Task Execution in LANS and Performance Analysis. Conference Proceedings - Int'l Phoenix Conf. on Computers and Comm. (1995), New Piscataway, NJ, USA, 423-429.

[11] Leutenegger, S.T. and Sun, X. Distributed Computing Feasibility in a NonDedicated Homogeneous Distributed Systems. IEEE Proc. Supercomputing '93, (November 1993).

[12] Mutka, M.W. Estimating Capacity For Sharing in a Privately Owned Workstation Environment. IEEE Trans. on Software Eng. 18, 4(April 1992), 319-328.

[13] Sun, X. and Rover, D. Scalability of Parallel Algorithm-Machine Combinations. IEEE Trans. Parallel Distribut. Systems. 5, 6(June 1994), 599-613.

[14] Nussbaum, D. and Agarwal, A. Scalability of Parallel Machines. Comm. of ACM 34, 3(March 1991), 57-61. 
[15] Karp, A.H. and Flat, H.P. Measuring Parallel Processor Performance. Comm. of ACM 33, 5(May 1990), 539-543.

[16] Daasch, W.R., Schaefer, L.R. and Driscoll, M.A., A Coordination Framework f or Optimized Mapping of Coarse-Grained Applications to a Distributed System. Private Communication. (1991).

[17] Eager,D.L., Zahorjan, J. and Lazowska, E.D. Speedup Versus Efficiency in Parallel Systems. IEEE Trans. Computers 38, 3(March 1989), 408-423.

[18] Donaldson, V., Berman, F. and Paturi R. Program Speedup in a Heterogeneous Computing Network. J. of Parallel and Distr. Computing 21, (1994), 316-322.

[19] Nelson, R., Towsley, D., and Tantawi, A.N. Performance Analysis of Parallel Processing Systems. IEEE Trans. on Software Eng. 14, 4(April 1988), 532539.

[20] Heidelberger, P. and Trivedi, K.S. Queueing Network Models for Parallel Processing with Asynchronous Tasks. IEEE Trans. on Computers c-31, 11(November 1982), 1099-1108.

[21] Baccelli, F. and Liu, Z. On the Execution of Parallel Programs on Multiprocessor Systems - A Queuing Theory Approach. J. of the ACM 37, 2(April 1990), 373-414.

[22] Baccelli, F., Massey, W.A., and Towsley, D. Acyclic Fork-Join Queueing Networks. J. of the ACM 36, 3(July 1989), 615-642. 
[23] Kleinrock, L. Queuing Systems Volume 2: Computer Applications John Wiley \& Sons, New York, 1976

[24] Lazowska, E.D. et al. Quantitative System Performance Prentice Hall, Inc. New Jersey, 1984.

[25] Kleinrock, L. and Huang, J. On Parallel Processing Systems: Amdahl's Law Generilzed and Some Results on Optimal Design. IEEE Trans. on Software Eng. 18, 5 (May 1992), 134-147

[26] Hennessy, J.L. and Patterson, D.A. Computer Architecture A Quantitative Approach. Morgan Kaufman, San Mateo, CA, 1990.

[27] Gustafson, J.L. Reevaluating Amdahl's Law. Comm. of ACM 31, 2(May 1988), 532-533.

[28] Chamberlain, R.D. and Peterson, G.D. Beyond Execution Time: Expanding the Use of Performance Models. IEEE Parallel and Distr. Tech. (1994), 37-49.

[29] Driscoll, M. A. and Daasch, W. R. Accurate Predictions of Parallel Program Execution Time. J.Parallel Distribut. Comput. 25, 1(February 1995), 16-30.

[30] Zhang, X., Xu, Z. Multiprocessor Scalability Predictions Through Detailed Program Execution Analysis. Technical Report, High Performance Computing and Software Laboratory, U. of Texas at San Antonio

[31] Zhang, X., Yan, Y., and Ma, Q. Measuring and Analyzing Parallel Computing Scalability. Proc.94 Int'l Conf on Parallel Processing, CRC Press, Vol. II (August 1994). 
[32] Grama, A., Gupta, A., and Kumar, V. Isoefficiency Function: A Scalable Metric for Parallel Algorithms and Architectures. IEEE Parallel and Distributed Tech. 1, 3(August1993), 12-21.

[33] Zhang, X., Yan, Y., He, K. Latency Metric: AN Experimental Method for Measuring and Evaluating Parallel Program and Architecture Scalability. J. of Parallel and Distr. Computing 22, 3(1994), 392-410.

[34] Mechosa, C. R., Farrara, J.D. and Spahr, J.A. Achieving Superlinear Speedup on a Heterogeneous, Distributed System. IEEE Parallel \& Distrib. Tech. (Summer 1994), 57-61.

[35] Kumar, V., and Gupta, A. Analyzing Scalability of Parallel Algorithms and Architectures. J. of Parallel and Distr. Computing 22,3(1994), 379-391

[36] Wilson, G.V. A Glossery of Parallel Computing Terminology. IEEE Parallel and Distrib. Tech. 4,1(Febuary 1993), 52-67.

[37] Gupta, A. and Kumar. V. Performance Properties of Large Scale Parallel Systems. J. of Parallel and Distr. Computing 19, 3(November 1993), 234244.

[38] Gustafson, J.L. Response to once again Amdahl's Law. Comm. of the ACM 32, 2(Febuary 1989), 263-264.

[39] Girard, A. Routing and Dimensioning in Circuit-Switched Networks. AddisonWesley Publishing Company, Reading, Mass. 1991, 77-78. 
[40] Geist, A., Beguelin, A., Dongarra, J., Jiang, W., Mancheck, R., and Sunderam, V. PVM User's Guide and Reference Manual. Oak Ridge National Laboratory, September 1994.

[41] Merriam-Webster, A. Webster's Seventh Collegiate Dictionary, G. \& C. Merriam Company, Springfield, MA, 1967.

[42] Press, W.H., et al. Numerical Recipies in C, 2nd ed. Cambridge University Press, New York, 1994.

[43] Golub, G., and Ortega, J. Scientific Computing An Introduction with Parallel Computing, Academic Press, Boston, 1993. 


\section{Appendices}

\section{A. Job Pool Psuedocode}

This is for the A group, the B group joins group B and sets flag $=0$, other wise it is the same.

Join group A.

Set Flag $=1$.

Spawn copies of self.

AGET():

Access job pool A directory.

Get a "bundle" of n tasks.

Open log file in job pool A directory.

Update $\log$ file by subtracting number of tasks withdrawn from task number.

Close log file.

For each task:

Do work on the tasks.

Deliver each task to job pool B as finished.

Open $\log$ file for $\mathrm{B}$.

Read in task number, called numtaskB.

Update log file by adding one to the task number.

Open log file in job pool A. 
Read in task number, called numtaskA.

Close log file.

Find ratio $=$ numtask $A /($ numtask $A+$ numtaskB $)$.

If ratio $<.3$

Call BGET().

Else if ratio $>.7$

Call AGET().

Else if Flag $=1$

Call AGET().

Else

Call BGET().

End AGET()

BGET():

Access job pool B directory.

Get a "bundle" of $m$ tasks.

Open log file in job pool B directory.

Update log file by subtracting number of tasks withdrawn from task number.

Close $\log$ file.

For each task:

Do work on the tasks.

Deliver each task to done pool as finished. 
Open log file for the done pool.

Read in task number, called numdone.

Update $\log$ file by adding one to the task number.

Open log file in job pool B.

Read in task number, called numtaskB.

Close log file.

Determine tasks in A pool, numtask $\mathrm{A}=$ totaltask - numdone - numtaskB .

Find ratio $=$ numtaskB $/($ numtask $A+$ numtask $B)$.

If ratio $<.3$

Call ABGET().

Else if ratio $>.7$

Call BGET().

Else if Flag $==1$

Call AGET().

Else

Call BGET().

End BGET() 


\section{B. Experimental Program Runs}

\begin{tabular}{|c|c|c|c|c|c|}
\hline Run \# & Matrix Size & $\overline{G_{A}}$ & $\overline{G_{B}}$ & Processors & Notes \\
\hline 1 & $100 \times 100$ & 1 & 1 & $5 \times 1-5 \times 17$ & \\
\hline 2 & $50 \times 50$ & 1 & 1 & $5 \times 1-5 \times 17$ & \\
\hline 3 & $50 \times 50$ & 1 & 1 & $5 \times 1-5 \times 17$ & only 50 tasks total \\
\hline 4 & $50 \times 50$ & 10 & 10 & $5 \times 1-5 \times 11$ & \\
\hline 5 & $50 \times 50$ & 20 & 20 & $5 \times 1-5 \times 9$ & \\
\hline 6 & $50 \times 50$ & 30 & 30 & $5 \times 1-5 \times 7$ & \\
\hline 7 & $50 \times 50$ & 40 & 40 & $5 \times 1-5 \times 7$ & \\
\hline 8 & $50 \times 50$ & 50 & 50 & $5 X 1-5 X 5$ & \\
\hline 9 & $100 \times 100$ & 1 & 1 & $5 \times 1-5 \times 17$ & \\
\hline 10 & $100 \times 100$ & 100 & 100 & $5 \times 1-5 \times 17$ & \\
\hline 11 & $100 \times 100$ & 10 & 10 & $5 \times 1-5 \times 17$ & \\
\hline 12 & $100 \times 100$ & 20 & 20 & $5 \times 1-5 \times 17$ & \\
\hline 13 & $100 \times 100$ & 30 & 30 & $5 \times 1-5 \times 17$ & \\
\hline 14 & $100 \times 100$ & 40 & 40 & $5 \times 1-5 \times 17$ & \\
\hline 15 & $100 \times 100$ & 50 & 50 & $5 X 1-5 \times 17$ & \\
\hline 16 & $100 \times 100$ & 70 & 70 & $5 X 1-5 \times 17$ & \\
\hline 17 & $50 \times 50$ & 1 & 1 & $5 \times 2$ & additional info for 2 \\
\hline 18 & $50 \times 50$ & 10 & 10 & $5 \times 2$ & additional info for 4 \\
\hline 19 & $50 \times 50$ & 20 & 20 & $5 \times 2$ & \begin{tabular}{|l} 
additional info for 5 \\
\end{tabular} \\
\hline 20 & $50 \times 50$ & 30 & 30 & $5 \times 2$ & \begin{tabular}{|l} 
additional info for 6 \\
\end{tabular} \\
\hline 21 & $50 \times 50$ & 40 & 40 & $5 \times 2$ & additional info for 7 \\
\hline 22 & $50 \times 50$ & 50 & 50 & $5 \times 2$ & additional info for 8 \\
\hline 23 & $50 \times 50$ & 10 & 10 & $5 \times 10$ & additional info for 4 \\
\hline 24 & $50 \times 50$ & 30 & 30 & $5 \times 4$ & \begin{tabular}{|l} 
additional info for 6 \\
\end{tabular} \\
\hline 25 & $50 \times 50$ & 1 & 1 & $5 X 1-5 X 5$ & uneven pools \\
\hline 26 & $50 \times 50$ & 1 & 1 & $5 \times 7-5 \times 11$ & uneven pools \\
\hline 27 & $50 \times 50$ & 1 & 1 & \begin{tabular}{|l}
$5 \times 13-5 \times 17$ \\
\end{tabular} & \begin{tabular}{|l} 
uneven pools \\
\end{tabular} \\
\hline 28 & $50 \times 50$ & 20 & 1 & $5 X 1-5 X 5$ & uneven pools \\
\hline 29 & $50 \times 50$ & 20 & 1 & $5 \times 7-5 \times 11$ & \begin{tabular}{|l} 
uneven pools \\
\end{tabular} \\
\hline 30 & $50 \times 50$ & 20 & 1 & $5 \times 13-5 \times 17$ & \begin{tabular}{|l} 
uneven pools \\
\end{tabular} \\
\hline 31 & $50 \times 50$ & 1 & 20 & $5 X 1-5 X 5$ & uneven pools \\
\hline 32 & $50 \times 50$ & 1 & 20 & $5 \times 7-5 \times 11$ & uneven pools \\
\hline 33 & $50 \times 50$ & 1 & 20 & $5 \times 13-5 \times 17$ & uneven pools \\
\hline 34 & $50 \times 50$ & 1 & 50 & $5 X 1-5 \times 5$ & \begin{tabular}{|l} 
uneven pools \\
\end{tabular} \\
\hline 35 & $50 \times 50$ & 1 & 50 & $5 \times 7-5 \times 11$ & uneven pools \\
\hline
\end{tabular}




\begin{tabular}{|l|l|l|l|l|l|}
\hline 36 & $50 \times 50$ & 1 & 50 & $5 \times 13-5 \times 17$ & uneven pools \\
\hline 37 & $50 \times 50$ & 50 & 1 & $5 \times 1-5 \times 5$ & uneven pools \\
\hline 38 & $50 \times 50$ & 50 & 1 & $5 \times 7-5 \times 11$ & uneven pools \\
\hline 39 & $50 \times 50$ & 50 & 1 & $5 \times 13-5 \times 17$ & uneven pools \\
\hline
\end{tabular}

